EURANDOM PREPRINT SERIES

2012-006

April 19, 2012

Robust Portfolio Choice and Indifference Valuation

R.J.A. Laeven, M.A. Stadje

ISSN 1389-2355 


\title{
Robust Portfolio Choice and Indifference Valuation*
}

\author{
Roger J. A. Laeven \\ Dept. of Quantitative Economics \\ University of Amsterdam, EURANDOM \\ and CentER \\ R. J.A.Laeven@uva.nl
}

\author{
Mitja A. Stadje \\ Dept. of Econometrics and Operations Research \\ Tilburg University \\ and CentER \\ M.A.Stadje@uvt.nl
}

This Version: April 19, 2012

\begin{abstract}
We solve, theoretically and numerically, the problems of optimal portfolio choice and indifference valuation in a general continuous-time setting. The setting features (i) ambiguity and ambiguity averse preferences, (ii) discontinuities in the asset price processes, with a general and possibly infinite activity jump part next to a continuous diffusion part, and (iii) general and possibly non-convex trading constraints. We characterize our solutions as solutions to Backward Stochastic Differential Equations (BSDEs). We prove existence and uniqueness of the solution to the general class of BSDEs with jumps having a drift (or driver) that grows at most quadratically, encompassing the solutions to our portfolio choice and valuation problems as special cases. We provide an explicit decomposition of the excess return on an asset into a risk premium and an ambiguity premium, and a further decomposition into a piece stemming from the diffusion part and a piece stemming from the jump part. We further compute our solutions in a few examples by numerically solving the corresponding BSDEs using regression techniques.
\end{abstract}

Keywords: Robust preferences; Convex risk measures; Portfolio choice; BSDEs; Incomplete markets; Indifference valuation; Exponential utility; Relative entropy.

AMS 2010 Classification: Primary: 91B06, 91B16, 91B30; Secondary: 60E15, $62 \mathrm{P} 05$.

JEL Classification: D81, G10, G20.

${ }^{*}$ We are very grateful to Freddy Delbaen, Alexander Schied and seminar and conference participants at the EURANDOM Lecture Day on Financial Stochastics (December 2010), Tilburg (June 2011), Trieste (June 2011), the EURANDOM-ISI Workshop on Actuarial and Financial Statistics (August 2011) and Humboldt University Berlin (December 2011), for their comments and suggestions. This research was funded in part by the Netherlands Organization for Scientific Research (Laeven) under grant NWO VIDI 2009. 


\section{Introduction}

Two main problems in asset pricing are portfolio choice and valuation in incomplete markets. The study of the dynamic portfolio choice problem goes back to Merton [54, 55] who approached it using stochastic control theory. It has since been considered by numerous authors in a wide variety of settings. Contributions relevant to the setting considered in this paper include Cvitanic and Karatzas [17], who prove existence and uniqueness of the solution (optimal portfolio) to the utility maximization problem in a Brownian filtration when restricting investment strategies to convex sets; and Kallsen [46], who solves the continuous-time utility maximization problem in a market where asset prices follow exponential Lévy processes, both using the duality or martingale approach. For further references, see the review of Schachermayer [69].

A widely adopted method for valuation in incomplete markets is indifference valuation (Carmona [10]). It is related to the portfolio choice problem: under indifference valuation, the price of a claim is such that the agent is indifferent between selling and not selling the claim, provided that each of the two alternatives is combined with an optimal portfolio choice that maximizes utility. Particularly popular is exponential indifference valuation due to its analytical tractability on the one hand - the exponential form induces a convenient translation invariance property - and its theoretically appealing properties, especially in a dynamic context, on the other (El Karoui and Rouge [24], Delbaen et al. [18], Kabanov and Stricker [42], Mania and Schweizer [53]). See also Hu, Imkeller and Müller [38], Becherer [4], Morlais [57, 58], and Cheridito and $\mathrm{Hu}$ [12] for recent work on the problems of portfolio choice and indifference valuation.

Many decision-making problems, including asset pricing problems, involve ambiguity (probabilities unknown) and it is important to distinguish them from decision-making problems under risk (probabilities given). This distinction is theoretically meaningful and has also been empirically relevant since the Ellsberg [25] paradox. A rich class of models for decision-making under ambiguity is that of variational preferences (Maccheroni, Marinacci and Rustichini [51]). It includes the popular maxmin expected utility of Gilboa and Schmeidler [30], also referred to as multiple priors, and the multiplier preferences of Hansen and Sargent [34, 35] as special cases. Under ambiguity, the true probabilistic model is unknown to the decision maker (model uncertainty); approaches that explicitly account for the possibility that a specific probabilistic model may not be correct but only an approximation, are commonly referred to as robust approaches.

Recently, there has been a growing interest in the effects of ambiguity on portfolio choice and valuation; see, for example, Chen and Epstein [11], Lazrak and Quenez [48], Maenhout [52], Müller [59], Schied [70], Klöppel and Schweizer [45], Föllmer, Schied and Weber [28], Owari [61] and Sircar and Sturm [71]. The importance of incorporating ambiguity in the problems of portfolio choice and valuation is not merely theoretical as ambiguity plays a potential role in addressing important failures of purely risk-based settings that rule out model uncertainty. Examples of such failures include the equity premium puzzle and the home-bias puzzle (Chen and Epstein, [11]). However, all above-mentioned papers featuring ambiguity are restricted to a continuous Brownian setting and do not allow for any discontinuities (jumps) in the asset price processes.

In this paper we solve, theoretically and numerically, the two canonical optimization problems of portfolio choice and indifference valuation, under ambiguity and fairly general ambiguity 
averse preferences, and in a further general continuous-time setting: besides a continuous diffusion component, we allow for a general and possibly infinite activity jump component in the asset price processes, and for general and possibly non-convex trading constraints regarding buying and short-selling. As regards the ambiguity averse preferences, we assume that the economic agent exhibits certain variational preferences. By the nature of jumps, the jump component of a semi-martingale asset price dynamics model is not unlikely to be exposed to model risk and with such a model a setting allowing for ambiguity and ambiguity averse preferences seems particularly appealing.

We prove that the solutions to the optimal portfolio choice and valuation problems can be characterized as solutions to Backward Stochastic Differential Equations (BSDEs). As a by-product, which is of interest in its own right, we prove existence and uniqueness of the solution to the general class of BSDEs with jumps having a drift (or driver) that grows at most quadratically, encompassing the solutions to our portfolio choice and valuation problems as special cases. Essentially, this by-product generalizes existence and comparison results by Kobylanski [46], for BSDEs with at most quadratic growth in a Brownian filtration, to a general and possibly infinite activity jump setting. We also provide an economic interpretation to the optimal solutions and to the excess return on an asset, which we explicitly decompose into a risk premium and an ambiguity premium, and further decompose into a piece stemming from the diffusion part and a piece stemming from the jump part. We finally provide a numerically tractable procedure to compute our solutions (by numerically solving the corresponding BSDEs using regression techniques) and implement this procedure in a few examples.

A BSDE may be seen as a dynamic programming principle in a continuous-time stochastic setting. BSDEs play an important role in stochastic control; see, for example, Pardoux and Peng [62], Duffie and Epstein [22], El Karoui, Peng and Quenez [23], Chen and Epstein [11], Lazrak and Quenez [48], Skiadas [72], Lim [49, 50], Hamadène and Jeanblanc [33], Horst and Müller [36], and also the early work of Bismut [7]. In a Markovian setting, BSDEs correspond to semi-linear PDEs. As is well-known, the solution to a utility maximization problem with a numerical preference representation specified by a BSDE can in turn be characterized as a solution to a BSDE; see Klöppel and Schweizer [45] and Sircar and Sturm [71] for recent applications of this technique to portfolio choice and indifference valuation in a purely Brownian setting. Therefore, a standard approach in utility maximization has been to try converting the utility maximization problem into a 'BSDE type' stochastic control problem. One of the advantages of this approach to portfolio choice is that, contrary to static duality methods, BSDEs can also deal with non-convex trading constraints. Another advantage of using BSDEs is that their solutions can be computed numerically efficiently by Monte Carlo simulation.

Applications of BSDEs to utility maximization problems in incomplete markets in a Brownian setting include (with exponential, logarithmic or power utility) $\mathrm{Hu}$, Imkeller and Müller [38], Cheridito and $\mathrm{Hu}$ [12], and (with a general utility function) Horst et al. [37]; for a setting with continuous filtration or non-continuous filtration (but with exponential utility), see Mania and Schweizer [53], Morlais [57] and Becherer [4]. Morlais [58] generalizes some of these results adopting an exponential utility function and allowing for infinite activity jumps in the asset price processes, in a purely risk-based setting without ambiguity. In particular, she proves existence and uniqueness results for a special quadratic BSDE. Mathematically, we generalize parts of her and Becherer's [4] results by proving existence and uniqueness results for all possibly infinite activity jump BSDEs with a driver function that grows at most quadratically. Contrary to Morlais [58], Becherer [4] and Kobylanski [46], who prove their results by solving 
the primal problem, we use a duality approach, generalizing parts of the methods developed by Delbaen, Hu and Bao [19] in a Brownian filtration.

There are only few works studying the portfolio choice and valuation problems in a setting with jumps and ambiguity. Bordigoni, Matoussi and Schweizer [9] study ambiguity using the relative entropy and Jeanblanc, Matoussi and Ngoupeyou [41] generalize this work to a noncontinuous filtration, assuming a one-point jump distribution. Björk and Slinko [8] study asset prices with jumps in a non-utility framework, using different possible pricing kernels to obtain good-deal bound prices. Independently of our work, Delong [21] and Øksendal and Sulem [60] have recently also considered model uncertainty in continuous-time jump settings. In Delong [21], the portfolio choice problem is solved in a setting with a degenerate, one-point jump distribution in case of a linear utility and multiple priors preferences. Øksendal and Sulem [60] study the problem of maximizing exponential utility with finite activity (Poissonian) jumps, using a generalization of multiple priors different from variational preferences. Assuming that the solutions of certain BSDEs exist and that comparison principles hold, they derive optimality conditions under assumptions different from ours. We do not only characterize the solutions to the portfolio choice and indifference valuation problems in a general continuous-time setting, but also provide new existence and uniqueness results for solutions to the corresponding class of BSDEs. To the best of our knowledge, we are not aware of other work on the problems of portfolio choice and indifference valuation that allows for a comparable degree of generality for all these features - ambiguity, jumps, and general trading constraints - together.

It is known that in a setting without ambiguity, discontinuities (jumps) in the asset price process have a discernible impact on the optimal portfolio choice (Kallsen [46], Aït-Sahalia, Cacho-Diaz and Hurd [1]). This impact is especially prevalent when allowing for dependencies between the jumps, limiting the benefits of international diversification, whence providing a (further) possible explanation for the empirically observed home-bias in investors' portfolios. The impact of constraints on buying and short-selling is documented in a rich literature (see Rubinstein [66] for a review) and it is important to account for such trading constraints in the most general fashion.

This paper is organized as follows. In Section 2, we introduce the basic setting and review some preliminaries for BSDEs. In Section 3, we specify in further detail the economic agent's preferences. In Section 4, we state the dynamic optimization problems, characterize their solutions and prove existence and uniqueness of these solutions. Section 5 examines the decomposition of the excess return on an asset. Section 6 discusses and illustrates the numerical implementation in some examples. Proofs are collected in the Appendix.

\section{Setting and Preliminaries}

\subsection{Asset Return Dynamics, Trading Constraints and Preferences}

We consider an economic agent with initial wealth $w_{0}$, which he can invest in a risk-less bond and risky assets. At a given maturity time $T$, the agent is endowed with an additional payoff $F$. A classical problem in asset pricing is the question of how the agent should determine his optimal investment strategy. To answer this question, one first needs to address the following issues: (i) How to model the dynamics of the risky assets? (ii) Which constraints to impose on the trading strategies allowed? (iii) How to evaluate the quality of the agent's investment strategy? This section describes our approach to these issues. 
For the dynamics of the risky assets, we assume a continuous-time setting with a general and possibly infinite activity jump component next to a general continuous diffusion component with stochastic volatility, and ambiguity. Large jumps in asset prices represent major financial economic shocks, such as market crashes, shocks resulting from unexpected announcements of the FED, or environmental disasters causing sudden movements in prices. Ambiguity, which is sometimes also referred to as model risk or model uncertainty, means that the 'true' probabilistic model is unknown to the decision maker. A setting featuring ambiguity seems particularly appealing when allowing for jumps in asset prices: large jumps are inherently rare and the jump component of the model may therefore easily be subject to model risk (uncertainty).

Formally, we consider a filtered probability space $\left(\Omega, \mathcal{F},\left(\mathcal{F}_{t}\right)_{t \in[0, T]}, P\right)$ equipped with two independent stochastic processes:

(i) A standard $d$-dimensional Brownian motion $W$.

(ii) A real-valued point process $p$ defined on $[0, T] \times \Omega \times \mathbb{R} \backslash\{0\}$. We denote by $N_{p}(d s, d x)$ the associated counting measure (or random measure). (We will usually suppress $\omega$ in the notation.) We assume its compensator (or mean or intensity measure) $\hat{N}_{p}(d s, d x)$ to be of the form

$$
\hat{N}_{p}(d s, d x)=n_{p}(s, d x) d s .
$$

We suppose that the predictable measure $n_{p}(s, d x)$ is non-negative for every $s \in[0, T]$, and that for every $\epsilon>0$

$$
\left\|\sup _{s} n_{p}(s, \mathbb{R} \backslash\{(-\epsilon, \epsilon)\})\right\|_{\infty}<\infty
$$

where $\|\cdot\|_{\infty}$ denotes the norm given by the (essential) supremum over all $\omega$. Furthermore, we suppose that

$$
\left\|\sup _{s} \int_{\mathbb{R} \backslash\{0\}}\left(|x|^{2} \wedge 1\right) n_{p}(s, d x)\right\|_{\infty}<\infty .
$$

We assume that the filtration $\left(\mathcal{F}_{t}\right)_{t \in[0, T]}$ is the completion of the filtration generated by $W$ and $N_{p}$. We denote by $\mathcal{P}$ the predictable $\sigma$-algebra on $[0, T] \times \Omega$ with respect to $\left(\mathcal{F}_{t}\right)$. Let $\tilde{N}_{p}(d s, d x):=N_{p}(d s, d x)-\hat{N}_{p}(d s, d x)$.

Financial economic shocks arrive at discrete points in time. Every shock comes with a 'marker' $x . N_{p}(s, d x)$ is one if there is a shock at time $s$ with marker $x$ and $n_{p}(s, d x) d s$ is the expected number of shocks with size 'around' $x$ per time unit 'around' time $s$. All our results also hold for multi-dimensional markers.

We assume that the financial market consists of a risk-free bond with interest rate normalized to zero, and $n \leq d$ stocks. The price process of stock $i$, denoted by $S^{i}$, evolves according to the semi-martingale dynamics

$$
\frac{d S_{t}^{i}}{S_{t-}^{i}}=b_{t}^{i} d t+\sigma_{t}^{i} d W_{t}+\int_{\mathbb{R} \backslash\{0\}} \beta_{t}^{i}(x) \tilde{N}_{p}(d t, d x), \quad i=1, \ldots, n,
$$

where $b^{i}\left(\sigma^{i}, \beta^{i}\right)$ are $\mathbb{R}\left(\mathbb{R}^{1 \times d}, \mathbb{R}\right)$-valued, predictable and uniformly bounded stochastic processes. $b^{i}$ is commonly referred to as the excess return: the holder of a risky asset should be compensated for the risk he is bearing. The second term in (2.1) represents noise due to 
'normal' market movements and is locally Gaussian. We assume that $\sigma$ has full rank and $\sigma \sigma^{\top}$ is uniformly elliptic, i.e., $\varepsilon I_{n} \preceq \sigma_{t} \sigma_{t}^{\top} \preceq \hat{K} I_{n}$, for some constants $\hat{K}>\varepsilon>0$. The third term in (2.1) represents the dynamics due to financial economic shocks. $\beta^{i}(x)$ is the impact (jump size) of a shock with 'marker' $x$ on the asset price $S^{i}$. We assume that $\beta^{i}$ is larger than -1 to ensure positivity of $S^{i}, i=1, \ldots, n$. We further assume that $\beta^{i} \in L^{2, \infty}, i=1, \ldots, n$, where

$L^{2, \infty}=\left\{\tilde{H} \mid \tilde{H}\right.$ is $\mathcal{P} \otimes \mathbb{B}(\mathbb{R} \backslash\{0\})$ measurable and $\left.\left\|\sup _{s} \int_{\mathbb{R} \backslash\{0\}}\left|\tilde{H}_{s}(x)\right|^{2} n_{p}(s, d x)\right\|_{\infty}<\infty\right\}$.

This condition is satisfied, for instance, if $\left|\beta_{s}(x)\right| \leq K|x|$ for $|x|$ small. If $n<d$, the market is incomplete. If $n=d$, the market is typically still incomplete because of the jump component of the model. Note that if $\left\|\sup _{s} n_{p}(s, \mathbb{R} \backslash\{0\})\right\|_{\infty}<\infty$ would hold, so that we only have finitely many jumps, $S$ may be written as a standard jump diffusion model, originating from Merton [56]. In this model, asset returns evolve according to

$$
\frac{d S_{t}^{i}}{S_{t-}^{i}}=b_{t} d t+\sigma_{t} d W_{t}+\sum_{i} I_{\left\{T_{i}=t\right\}} J_{i}
$$

for jump times $T_{1}, T_{2}, \ldots$ with corresponding jump sizes $J_{1}, J_{2}, \ldots$.

For $i=1, \ldots, n$, the process $\pi_{t}^{i}$ represents the amount of capital invested in stock $i$ at time $t$, and the number of shares is $\frac{\pi_{t}^{i}}{S_{t}^{i}}$. The wealth process $X^{(\pi)}$ of a predictable trading strategy $\pi$ with initial capital $w_{0}$ satisfies

$$
X_{t}^{(\pi)}=w_{0}+\sum_{i=1}^{n} \int_{0}^{t} \frac{\pi_{u}^{i}}{S_{u-}^{i}} d S_{u}^{i}=w_{0}+\int_{0}^{t} \pi_{u}\left(\sigma_{u} d W_{u}+b_{u} d u\right)+\int_{0}^{t} \int_{\mathbb{R} \backslash\{0\}} \pi_{u} \beta_{u}(x) \tilde{N}_{p}(d u, d x) .
$$

We assume that the agent is allowed to choose trading strategies taking values in a compact and possibly non-convex set $U \subset \mathbb{R}^{1 \times n}$ a.s. We call $\pi$ an admissible trading strategy if it is predictable and takes values only in $U$. We denote the set of all admissible trading strategies by $\mathcal{A}$. Since the set $U$ is compact, for every trading strategy $\pi$, the wealth process $\sup _{t}\left|X_{t}^{(\pi)}\right|$ is square-integrable. By our assumptions, there exists a local martingale measure $Q^{f}$ under which $W_{t}-\int_{0}^{t} \sigma_{s}^{\top}\left(\sigma_{s} \sigma_{s}^{\top}\right)^{-1} b_{s} d s$ is a Brownian motion (and hence $S$ is a local martingale). In particular, there is no arbitrage in the market.

The agent, choosing an investment strategy $\left(\pi_{t}\right)$ and being endowed with a payoff $F$, eventually holds the portfolio $F+X_{T}^{(\pi)}$ at maturity. The final issue to be addressed is which decision criterion to use when evaluating the quality of the agent's portfolio choice. The classical decision criterion in a setting featuring ambiguity is Savage's [68] subjective expected utility; it postulates that the economic agent specifies a subjective probability measure $P$ and a utility function $u$, and evaluates the portfolio according to $U\left(F+X_{T}^{(\pi)}\right)=\mathrm{E}\left[u\left(F+X_{T}^{(\pi)}\right)\right]$. We note that specifying the measure $P$ in our setting implies specifying (estimating) the excess return $b_{t}$, the Gaussian volatility $\sigma_{t}$, and the impact of the jumps $\beta_{t}(x) n(t, d x)$ : a challenging econometric exercise. It is well-known in decision theory, starting with the Ellsberg paradox [25], that, faced with ambiguity, agents tend to make decisions that are inconsistent with subjective expected utility.

Various alternative approaches to decision-making under ambiguity have emerged in the literature. Among the best-known alternatives is multiple priors, of Gilboa and Schmeidler 
[30] (see also Wald [73] and Huber [39]); it postulates that an economic agent evaluates his portfolio according to $U\left(F+X_{T}^{(\pi)}\right)=\inf _{Q \in M} \mathrm{E}_{Q}\left[u\left(F+X_{T}^{(\pi)}\right)\right]$, where $M$ is a set of probabilistic models (or priors). Multiple priors was significantly generalized by Maccheroni, Marinacci, and Rustichini [51] to the theory of variational preferences, postulating that an economic agent evaluates his portfolio according to

$$
U\left(F+X_{T}^{(\pi)}\right)=\inf _{Q \in \mathcal{Q}}\left\{\mathrm{E}_{Q}\left[u\left(F+X_{T}^{(\pi)}\right)\right]-c(Q)\right\} .
$$

Variational preferences go beyond multiple priors preferences by allowing to attach a plausibility index $c$ (the penalty function) to every probabilistic model $Q$ in the class of probabilistic models $\mathcal{Q}$ under consideration. If $c(Q)=\infty$, the minimum in (2.2) is not attained in this particular $Q$, meaning that probabilistic models with infinite penalty are considered fully unreliable and are effectively excluded from the analysis. Multiple priors occurs when $c(Q)=I_{M}$, the penalty function that is zero if $Q \in M$ and $\infty$ otherwise, attaching the same plausibility to all probabilistic models in $M$. In the case that $u$ is linear, multiple priors corresponds to coherent risk measures (Artzner et al. [2]) and variational preferences corresponds to convex risk measures (Föllmer and Schied [26], Frittelli and Rosazza Gianin [29], Ruszczyński and Shapiro [67]); see Laeven and Stadje [47] for further results on these connections.

We solve the portfolio choice and valuation problems, adopting certain versions of variational preferences (2.2). To distinguish between $U(\cdot)$ and $u(\cdot)$, we call $U$ an evaluation and $u$ a utility function. In our continuous-time setting, we (need to) consider the dynamic version of $(2.2)$, which is given by

$$
U_{t}\left(F+X_{T}^{(\pi)}\right):=\operatorname{essinf}_{Q \in \mathcal{Q}}\left\{\mathrm{E}_{Q}\left[u\left(F+X_{T}^{(\pi)}\right) \mid \mathcal{F}_{t}\right]-c_{t}(Q)\right\},
$$

in which $c_{t}(Q)$ reflects the esteemed plausibility of the model $Q$ given the information up to time $t$. The portfolio choice problem is then given by $V_{t}(F)=\max _{\pi \in \mathcal{A}} U_{t}\left(F+X_{T}^{(\pi)}\right)$, at time $t$.

The class of all alternative probabilistic models considered is specified as

$$
\mathcal{Q}=\{Q \mid \text { If for an event } A: P(A)=0, \text { then also } Q(A)=0\}=\{Q \mid Q \ll P\},
$$

i.e., all measures $Q$ that are absolutely continuous with respect to the reference model $P$ are considered; sets with probability zero under the reference model $P$ still have probability zero under the alternative model $Q$. It means, for example, that if, with probability one under $P$, the financial asset has only finitely many jumps, it also has only finitely many jumps under every $Q$. We subsequently assume that $u$ is linear, exponential or logarithmic. In the case that $u$ is linear or exponential, the problem is translation invariant, in particular, the optimal hedge is independent of the wealth of the agent. In this case we can also explicitly calculate the indifference valuation. We further consider penalty functions for which a dynamic programming principle is satisfied; see Section 3 for further details.

\section{$2.2 \quad$ BSDEs}

We solve the portfolio choice and indifference valuation problems using backward stochastic differential equations (BSDEs). We denote by $|\cdot|$ the Euclidean norm and by $\mathcal{S}^{\infty}$ the class of all one-dimensional $\left(\mathcal{F}_{t}\right)$-adapted semi-martingales $X$ which are bounded. Define $|X|_{S^{\infty}}=$ 
$\left\|\sup _{t}\left|X_{t}\right|\right\|_{\infty}$. Let

$$
\mathcal{H}^{2}:=\left\{Z=\left(Z^{1}, \ldots, Z^{d}\right) \mid Z^{i} \in \mathcal{P} \text { for } i=1, \ldots, d \text { and } \mathrm{E}\left[\int_{0}^{T}\left|Z_{s}\right|^{2} d s\right]<\infty\right\} .
$$

Furthermore, we denote by $L^{2}\left(d P \times n_{p}(s, d x) \times d s\right)$ all functions measurable with respect to $\mathcal{P} \otimes \mathcal{B}(\mathbb{R} \backslash\{0\})$ which are square-integrable with respect to $d P \times n_{p}(s, d x) \times d s$.

A solution to the (one-dimensional) BSDE with driver $g(t, z, \tilde{z})$ mapping to $\mathbb{R}$ and terminal condition $F \in L^{\infty}\left(\mathcal{F}_{T}\right)$ is a triple of processes $(Y, Z, \tilde{Z}) \in \mathcal{S}^{\infty} \times \mathcal{H}^{2} \times L^{2}\left(d P \times n_{p}(s, d x) \times d s\right)$ such that

$$
d Y_{t}=g\left(t, Z_{t}, \tilde{Z}_{t}\right) d t-Z_{t} d W_{t}-\int_{\mathbb{R} \backslash\{0\}} \tilde{Z}_{t}(x) \tilde{N}_{p}(d t, d x) \text { and } Y_{T}=F .
$$

In order for BSDEs to be well-defined, $g$ needs to satisfy certain measurability conditions; see the Appendix. Often times BSDEs are written in the following equivalent form:

$$
Y_{t}=F-\int_{t}^{T} g\left(s, Z_{s}, \tilde{Z}_{s}\right) d s+\int_{t}^{T} Z_{s} d W_{s}+\int_{t}^{T} \int_{\mathbb{R} \backslash\{0\}} \tilde{Z}_{s}(x) \tilde{N}_{p}(d s, d x) .
$$

Since the terminal condition is given at maturity time $T$, BSDEs have to be computed backwards in time, whence their name. As in many applications a terminal reward is specified, and solutions of BSDEs satisfy a dynamic programming principle, BSDEs are often applied to solve problems in stochastic control and mathematical finance; see the references provided in the Introduction.

It is well-known that if $g(t, 0,0)$ is in $L^{\infty}(d P \times d t)$ and $g$ is further uniformly Lipschitz continuous, that is, there exists $K>0$ such that

$$
\left|g\left(t, z_{1}, \tilde{z}_{1}\right)-g\left(t, z_{0}, \tilde{z}_{0}\right)\right| \leq K\left(\left|z_{1}-z_{0}\right|+\sqrt{\int_{\mathbb{R} \backslash\{0\}}\left|\tilde{z}_{1}(x)-\tilde{z}_{0}(x)\right|{ }^{2} n_{p}(t, d x)}\right),
$$

then a unique solution to the corresponding BSDE exists; see, for example, Royer [65]. However, we will encounter BSDEs with drivers that grow quadratically. Therefore, new analytical and numerical tools need to be developed.

Example 2.1 Let $F$ be a bounded payoff and define $Y_{t}=\mathrm{E}\left[F \mid \mathcal{F}_{t}\right]$. Then, $Y_{T}=F$. Moreover, by the martingale representation theorem (see, e.g., Jacod and Shiryaev [40], Ch. 3, Sec. 4) there exist predictable processes $Z$ and $\tilde{Z}$ such that $Y$ satisfies

$$
d Y_{t}=-Z_{t} d W_{t}-\int_{\mathbb{R} \backslash\{0\}} \tilde{Z}_{t}(x) \tilde{N}_{p}(d t, d x) .
$$

This is the simplest BSDE with $g=0$.

Hence, a conditional expectation may be seen as a BSDE with $g=0$. It explains why BSDEs are also being referred to as $g$-expectations. The name should express that a BSDE may be viewed as a generalized (usually non-linear) conditional expectation with an additional drift. 
Example 2.2 Let $F$ be a bounded payoff and define $Y_{t}=\mathrm{E}_{Q^{f}}\left[F \mid \mathcal{F}_{t}\right]$. Then by the martingale representation theorem and the Lenglart-Girsanov theorem (Jacod and Shiryaev [40], Ch. 3, Th. 3.11) $Y$ satisfies

$$
d Y_{t}=-Z_{t} \sigma_{t}^{\top}\left(\sigma_{t} \sigma_{t}^{\top}\right)^{-1} b_{t} d t-Z_{t} d W_{t}-\int_{\mathbb{R} \backslash\{0\}} \tilde{Z}_{t}(x) \tilde{N}_{p}(d t, d x) \text { and } Y_{T}=F .
$$

This is a linear BSDE with $g(t, z, \tilde{z})=-z \sigma_{t}^{\top}\left(\sigma_{t} \sigma_{t}^{\top}\right)^{-1} b_{t}$.

In a Markovian setting, $g$-expectations correspond to viscosity solutions to semi-linear parabolic PDEs (or PIDEs in the case of jumps); see, for example, El Karoui, Peng and Quenez [23] in a Brownian setting and Barles, Buckdahn and Pardoux [3] in the case of jumps. In particular, our results on BSDEs also give rise to results for viscosity solutions to the corresponding PIDEs in the case of infinite jump activity. However, because BSDEs are more general (since they do not rely on a Markovian structure) and because our numerical implementation is based on Monte Carlo simulation which relies on the structure of the BSDE, we believe that it is more useful to approach the problems under consideration using BSDEs rather than using PIDEs.

\section{Ambiguity Averse Preferences and Dynamic Programming}

We specify below assumptions on the plausibility index $c$ in (2.2)-(2.3) such that a dynamic programming principle is satisfied. For this purpose, let us first take a closer look at the set of alternative models $\mathcal{Q}=\{Q \mid Q \ll P\}$. It is well-known that in a Brownian filtration, every probability measure $Q$ absolutely continuous with respect to $P$ can be identified with a stochastic drift $q:[0, T] \times \Omega \rightarrow \mathbb{R}^{d}$ such that $W_{t}-\int_{0}^{t} q_{s} d s$ is a Brownian motion under $Q$. It means that in a Brownian filtration, the setting of ambiguity, in which a collection of probability measures (priors) is considered rather than a single probability measure, can be fully described by a collection of drifts $q$.

Now let us address the question of how to model ambiguity with respect to the jump component of the model. If $Q \in \mathcal{Q}$, we denote by $D_{t}$ the Radon-Nikodym derivative $D_{t}=$ $\mathrm{E}\left[\frac{d Q}{d P} \mid \mathcal{F}_{t}\right]$. One may see that there exist a predictable stochastic drift $q$ and a function $\psi$ : $[0, T] \times \Omega \times \mathbb{R} \backslash\{0\} \rightarrow[-1, \infty)$, measurable with respect to $\mathcal{P} \otimes \mathcal{B}(\mathbb{R} \backslash\{0\})$, such that the Radon-Nikodym derivative can be written as

$$
\begin{aligned}
D_{t}=\exp \left\{\int_{0}^{t} q_{s} d W_{s}-\frac{1}{2} \int_{0}^{t} \mid\right. & \left.q_{s}\right|^{2} d s+\int_{0}^{t} \int_{\mathbb{R} \backslash\{0\}} \psi_{s}(x) \tilde{N}_{p}(d s, d x) \\
& \left.+\int_{0}^{t} \int_{\mathbb{R} \backslash\{0\}}\left[\log \left(1+\psi_{s}(x)\right)-\psi_{s}(x)\right] N_{p}(d s, d x)\right\},
\end{aligned}
$$

for $t \leq \tau$ and $\tau=\inf \left\{t \mid D_{t}=0\right\} \wedge T$. In particular, $Q$ is uniquely characterized by $q$ and $\psi$.

Expression (3.1) is seen as follows: Clearly, the Radon-Nikodym derivative $D_{t}$ is a martingale. For $t \geq \tau$, we must have that $D_{t}=0$. Furthermore, the whole path of $D_{t-}$ is strictly positive up to time $\tau$, see Lemma A.11 in the Appendix. By Jacod and Shiryaev [40], Ch. 3, Sec. 4, there exist a locally integrable* process $H:[0, T] \times \Omega \rightarrow \mathbb{R}$, measurable with respect to

${ }^{*}$ See Definition A.2 in the Appendix. 
$\mathcal{P}$, and a locally integrable function $\tilde{H}:[0, T] \times \Omega \times \mathbb{R} \backslash\{0\} \rightarrow \mathbb{R}$, measurable with respect to $\mathcal{P} \otimes \mathcal{B}(\mathbb{R} \backslash\{0\})$, such that

$$
d D_{t}=H_{t} d W_{t}+\int_{\mathbb{R} \backslash\{0\}} \tilde{H}_{t}(x) \tilde{N}_{p}(t, d x),
$$

with $D_{0}=1$. For $t \geq \tau$, we must have that $H_{t}=0$ and $\tilde{H}_{t}=0$. Therefore, defining $0 / 0=0$, we obtain

$$
\begin{aligned}
d D_{t} & =D_{t-}\left(\frac{H_{t}}{D_{t-}} d W_{t}+\int_{\mathbb{R} \backslash\{0\}} \frac{\tilde{H}_{t}(x)}{D_{t-}} \tilde{N}_{p}(d t, d x)\right) \\
& =: D_{t-}\left(q_{t} d W_{t}+\int_{\mathbb{R} \backslash\{0\}} \psi_{t}(x) \tilde{N}_{p}(d t, d x)\right)
\end{aligned}
$$

for $t \leq \tau$. The solution to this SDE is given by the stochastic exponential $\mathcal{E}\left(\int_{0}^{t} q_{s} d W_{s}+\int_{0}^{t} \int_{\mathbb{R} \backslash\{0\}} \psi_{s}(x) \tilde{N}_{p}(d s, d x)\right)$, which equals the right-hand side in (3.1). The stochastic exponential is also referred to as the Doléans-Dade exponential.

Since $D_{t}$ is non-negative, we must have that $\psi \geq-1, d P \times n_{p}(t, d x) \times d t$-a.s. If $Q$ is equivalent to $P$, then, by the Lenglart-Girsanov theorem, $W_{t}^{Q}=W-\int_{0}^{t} q_{s} d s$ is a Brownian motion and the process $\tilde{N}$ has compensator $n^{Q}(s, d x):=\left(1+\psi_{s}(x)\right) n_{p}(s, d x)$, under $Q$ (see, for instance, Jacod and Shiryaev [40], Ch. 3, Th. 3.11). Consequently, $1+\psi$ is the new density of the jump component under $Q$. Hence, $q$ may be seen as an additional drift that the reference model $P$ may have failed to detect, and $\psi$ may be seen as a misspecification of the size and frequency of the jumps under $P$. (The model $P$ corresponds to $q=\psi=0$.)

A standard example of the plausibility index in (2.2) is the relative entropy (Csiszár [16], Ben-Tal [6]) defined as

$$
c(Q)=c_{0}(Q)=\alpha H(Q \mid P), \quad \alpha>0, \quad \text { with } \quad H(Q \mid P)=\left\{\begin{array}{l}
\mathrm{E}_{Q}\left[\log \left(\frac{d Q}{d P}\right)\right], \quad \text { if } Q \in \mathcal{Q} ; \\
\infty, \text { otherwise } .
\end{array}\right.
$$

The relative entropy is also known as the Kullback-Leibler divergence; it measures the distance between the distributions $Q$ and $P$. The relative entropy is used e.g., by Hansen and Sargent $[34,35]$ in the context of model robustness in macroeconomics. The interpretation is that the economic agent has a reference measure $P$, but the measure $P$ is merely an approximation to the probabilistic model rather than the true model. As such, the agent does not fully trust the measure $P$ and considers many measures $Q$, with esteemed plausibility decreasing proportionally to their distance from the approximation $P$. The parameter $\alpha$ may be viewed as measuring the degree of trust the agent puts in the reference measure $P$, with $\alpha=\infty$ corresponding to a maximal degree of trust, and $\alpha=0$ corresponding to a maximal degree of distrust.

In our setting, it may be seen that

$$
c(Q)=\alpha H(Q \mid P)=\mathrm{E}_{Q}\left[\int_{0}^{T}\left\{r_{1}\left(q_{s}\right)+\int_{\mathbb{R} \backslash\{0\}} r_{2}\left(\psi_{s}(x)\right) n(s, d x)\right\} d s\right],
$$


with $q$ and $\psi$ corresponding to the measure $Q$ according to (3.1), and

$$
r_{1}(q)=\frac{\alpha}{2}|q|^{2} \text { and } r_{2}(y)= \begin{cases}\alpha[(1+y) \log (1+y)-y], & \text { if } y \geq-1 \\ \infty, & \text { otherwise }\end{cases}
$$

see Proposition A.17 in the Appendix. Note that $r_{1}$ and $r_{2}$ are maximal at $q=0$ and $\psi=0$ with $r_{1}(0)=r_{2}(0)=0$. These values of $q$ and $\psi$ correspond to the probabilistic model $P$, hence, the reference model has the highest esteemed plausibility. Furthermore, the larger $|q|$ and $|\psi|$, the less plausible is the corresponding probabilistic model.

We generalize the relative entropy, by postulating that the penalty function $c$ must take the form of an integral of convex functions depending on the drift, $q$, and the intensity, $\psi$. That is, we henceforth assume:

(H1) $c_{t}$ is of the form

$$
c_{t}(Q)=\mathrm{E}_{Q}\left[\int_{t}^{T}\left[r_{1}\left(s, q_{s}\right)+\int_{\mathbb{R} \backslash\{0\}} r_{2}\left(s, x, \psi_{s}(x)\right) n_{p}(s, d x)\right] d s \mid \mathcal{F}_{t}\right],
$$

for functions $r_{1}:[0, T] \times \Omega \times \mathbb{R}^{d} \rightarrow \mathbb{R}_{0}^{+} \cup\{\infty\}$ and $r_{2}:[0, T] \times \Omega \times \mathbb{R} \times[-1, \infty) \rightarrow \mathbb{R}_{0}^{+} \cup\{\infty\}$ that are measurable with respect to $\mathcal{P} \otimes \mathbb{B}\left(\mathbb{R}^{d}\right)$ and $\mathcal{P} \otimes \mathbb{B}(\mathbb{R}) \otimes \mathbb{B}([-1, \infty))$, respectively. Moreover, for every $t, \omega, x$ we assume that $r_{1}(t, \cdot)$ and $r_{2}(t, x, \cdot)$ are convex and continuous on their domain ${ }^{\dagger}$ in $q$ and $\psi$, respectively, and $r_{1}(t, 0)=r_{2}(t, x, 0)=0$ for all $t, x$.

Note that for a convex function $f$ mapping from $\mathbb{R}^{k} \rightarrow \mathbb{R} \cup\{\infty\}$, continuity on the domain is equivalent with $f$ being lower-semicontinuous if $k=1$. However, in higher dimensions, continuity on the domain is stronger than lower-semicontinuity.

We have motivated condition (H1) by generalizing the frequently adopted relative entropy; however, an alternative motivation is based on the following. If $U$ is a monetary utility function (that is, an evaluation under variational preferences with a linear utility function modulo a change of signs), then Delbaen, Peng and Rosazza Gianin [20] show in a purely Brownian filtration that Bellman's principle is equivalent to $c_{t}$ being of the form

$$
c_{t}(Q)=\mathrm{E}_{Q}\left[\int_{t}^{T} r\left(s, q_{s}\right) d s \mid \mathcal{F}_{t}\right]
$$

with $r:[0, T] \times \Omega \times \mathbb{R}^{d} \rightarrow \mathbb{R}_{0}^{+} \cup\{\infty\}$ being convex and lower-semicontinuous in $q$. Since Bellman's principle seems necessary to tackle a dynamic programming problem in continuoustime, a condition like (H1), generalizing the form obtained by Delbaen, Peng and Rosazza Gianin [20] to a setting with jumps, seems to be unavoidable.

We want to consider penalty functions that can be bounded from below in terms of the relative entropy, i.e., there exist $K_{1}^{\prime}, K_{2}^{\prime}>0$ such that $c(Q) \geq-K_{1}^{\prime}+K_{2}^{\prime} H(Q \mid P)$. In view of (3.3), this corresponds to $r_{1}$ and $r_{2}$ satisfying the following growth condition, henceforth denoted by (H2): There exist constants $K_{1}, K_{2}>0$ and a bounded function $\left(\tilde{A}_{t}(\omega, x)\right)_{t, \omega, x} \in$ $L^{2, \infty}$ such that for all $t$, a.s. all $\omega$, all $q, x$ and $y$

$$
\begin{aligned}
r_{1}(t, \omega, q) & \geq-K_{1}+K_{2}|q|^{2}, \\
r_{2}(t, \omega, x, y) & \geq-\tilde{A}_{t}(\omega, x)+K_{2}\{(1+y) \log (1+y)-y\} .
\end{aligned}
$$

\footnotetext{
${ }^{\dagger}$ A convex function $f$ is continuous on its domain if for $x_{n}$ converging to $x$ with $f\left(x_{n}\right)<\infty$ for all $n \in \mathbb{N}$, $\lim _{n} f\left(x_{n}\right)=f(x)$.
} 
We need one more condition: we suppose that (sub)differentials of the penalty functions $r_{1}$ and $r_{2}$ can be bounded from below in terms of the differentials of the relative entropy. (The notion of subdifferentiability is a generalization of the usual definition of differentiability; it is frequently used for convex functions, see the Appendix for details.) That is, we henceforth assume:

(H3) There exist constants $\hat{K}_{1}, \hat{K}_{2}>0$, such that for all $t$, a.s. all $\omega$, and all $q$,

$$
\left|\partial_{q} r_{1}(t, \omega, q)\right| \geq-\hat{K}_{1}+\hat{K}_{2}|q| .
$$

Furthermore, for every $C>0$, there exist $\hat{K}_{3}>0$ and a bounded function $\left(\tilde{K}_{t}(\omega, x)\right)_{t, \omega, x} \in$ $L^{2, \infty}$, such that for all $t \in[0, T]$, a.s. all $\omega$, all $x \in \mathbb{R}$ and $y \in[-C, C]$,

$$
\left|\partial_{y} r_{2}(t, \omega, x, y)\right| \geq-\tilde{K}_{t}(\omega, x)+\hat{K}_{3}|\log (1+y)| .
$$

These inequalities should hold for every element of the corresponding subdifferential, where we set $|\emptyset|=\infty$.

In case the penalty function is the relative entropy with parameter $\alpha$, one can choose the constants in (H2)-(H3) as follows: $K_{1}=\hat{K}_{1}=\tilde{A}=\tilde{K}=0, K_{2}=\frac{\alpha}{2}$, and $\hat{K}_{2}=\hat{K}_{3}=\alpha$. Since (H2) is a rather slow growth condition (which is satisfied for most examples in the literature), the results presented in this paper are applicable to 'almost all' penalty functions. If the penalty function grows slower than the relative entropy, then, in a purely Brownian setting (without jumps), the corresponding (dual) superquadratic BSDEs we would derive, do not have a solution; see Delbaen, Hu and Bao [19]. Therefore, a growth condition like (H2) seems to be necessary for our approach, unless one would want to consider supersolutions of BSDEs.

\section{The Optimization Problems and Their Solutions}

\subsection{Linear Utility Under Variational Preferences}

At time $t$, we are interested in the following optimization problem:

$$
V_{t}(F)=\operatorname{ess}_{\sup } \operatorname{su\mathcal {A}}_{t} U_{t}\left(F+X_{T}^{(\pi)}\right)
$$

where $U_{t}$ is defined in (2.3) with plausibility index $c$ satisfying $(\mathrm{H} 1)-(\mathrm{H} 3), \mathcal{A}$ is the set of admissible trading strategies, $F$ is the (bounded) payoff at maturity and $X_{T}^{(\pi)}$ is the wealth process. We first assume that the utility function $u$ in (2.3) is linear. Then one may see from Lemma A.27 in the Appendix that, for every admissible $\pi, U_{t}\left(F+X_{T}^{(\pi)}\right)$ is finite. We solve problem (4.1) with the help of BSDEs. Define

$$
\begin{aligned}
g_{1}(t, z): & =\sup _{q \in \mathbb{R}^{d}}\left\{z q-r_{1}(t, q)\right\} \\
g_{2}(t, x, a): & =\sup _{y \in \mathbb{R}}\left\{y a-r_{2}(t, x, y)\right\},
\end{aligned}
$$

for $t \in[0, T], z \in \mathbb{R}^{1 \times d}$, and $a, x \in \mathbb{R}$. For a function $\tilde{z}: \mathbb{R} \backslash\{0\} \rightarrow \mathbb{R}$, define further $g(t, z, \tilde{z}):=g_{1}(t, z)+\int_{\mathbb{R} \backslash\{0\}} g_{2}(t, x, \tilde{z}(x)) n_{p}(t, d x)$. It follows from Lemma A.7 in the Appendix that $g_{i}$ are real-valued, suitably measurable, and $g_{i} \geq 0$ with equality if $z=0$ or $a=0, i=1,2$. 
So both functions $g_{i}$ assume their minimum at zero. The next theorem shows, as an auxiliary result, that, under our assumptions, $U(F)$ is the unique solution to a BSDE with terminal condition $F$ and driver function $g$.

Theorem 4.1 Assume that (H1)-(H3) hold. Then $U_{t}(F)$ is the unique solution to the BSDE

$$
\begin{aligned}
d U_{t}(F) & =\left[g_{1}\left(t, Z_{t}\right)+\int_{\mathbb{R} \backslash\{0\}} g_{2}\left(t, x, \tilde{Z}_{t}(x)\right) n_{p}(t, d x)\right] d t-Z_{t} d W_{t}-\int_{\mathbb{R} \backslash\{0\}} \tilde{Z}_{t}(x) \tilde{N}_{p}(d t, d x), \\
U_{T}(F) & =F .
\end{aligned}
$$

As a by-product, while proving this theorem, we show in the Appendix that every BSDE with driver functions $g_{1}$ and $g_{2}$, both growing at most quadratically, has a unique solution satisfying a comparison principle; see Theorem A.21, Remark A.23, and Proposition A.26.

If $g_{1}=g_{2}=0$ would hold, the evaluation $U$ would correspond to a conditional expectation; see Example 2.1 of Section 2.2. However, our economic agent is ambiguity averse, considering all alternative probabilistic models, with different degrees of esteemed plausibility. As a result, $g_{i} \geq 0$ for $i=1,2$, which decreases the evaluation. $Z$ is the stochastic (Malliavin) derivative of the evaluation with respect to $W$. Comparing (4.4) with (2.1), we see that $Z$ and $\tilde{Z}$ play the same role for $U(F)$ as $\sigma$ and $\beta$ for the instantaneous return of the asset price. Therefore, $Z$ and $\tilde{Z}$ may be seen as measuring the degree of fluctuation ('variability') of the evaluation coming from the Brownian motion and from the jumps, respectively. The larger $|Z|$, the more variability is due to the local Gaussian part, and the larger $|\tilde{Z}|$, the more variability is due to the jump component of the model.

Next, let

$$
f(s, z, \tilde{z}):=\inf _{\pi \in U}\left\{-\pi b_{s}+g_{1}\left(s, z-\pi \sigma_{s}\right)+\int_{\mathbb{R} \backslash\{0\}} g_{2}\left(s, x, \tilde{z}(x)-\pi \beta_{s}(x)\right) n_{p}(s, d x)\right\} .
$$

By Theorem A.24 in the Appendix, the BSDE

$$
Y_{t}=F-\int_{t}^{T} f\left(s, Z_{s}, \tilde{Z}_{s}\right) d s+\int_{t}^{T} Z_{s} d W_{s}+\int_{t}^{T} \int_{\mathbb{R} \backslash\{0\}} \tilde{Z}_{s}(x) \tilde{N}_{p}(d s, d x), \quad t \in[0, T],
$$

has a unique solution $(Y, Z, \tilde{Z})$. Then we state the following theorem:

Theorem 4.2 Assume that (H1)-(H3) hold. Then $V_{0}(F)=Y_{0}+w_{0}$ and the optimal strategy $\pi_{s}^{*}$ is a predictable process that attains the infimum in (4.5) for $(z, \tilde{z})=\left(Z_{s}, \tilde{Z}_{s}\right)$, i.e.,

$$
f\left(s, Z_{s}, \tilde{Z}_{s}\right)=-\pi_{s}^{*} b_{s}+g_{1}\left(s, Z_{s}-\pi_{s}^{*} \sigma_{s}\right)+\int_{\mathbb{R} \backslash\{0\}} g_{2}\left(s, x, \tilde{Z}_{s}(x)-\pi_{s}^{*} \beta_{s}(x)\right) n_{p}(s, d x) .
$$

Heuristically, the optimal portfolio choice proceeds as follows: The excess return, $b$, is typically positive. Hence, the term $-\pi b_{s}$ in the minimization problem (4.5) will 'tempt' the economic agent to invest in risky assets (that is, to pick a positive $\pi$ ) so as to benefit from the excess return. The agent is, however, ambiguity averse. Therefore $Z$, representing the variability of the evaluation due to the Brownian component, is penalized by $g_{1}\left(s, Z_{s}\right)$ (before hedging); and $\tilde{Z}$, representing the variability of the evaluation due to jumps, is penalized by $\int_{\mathbb{R} \backslash\{0\}} g_{2}\left(s, x, \tilde{Z}_{s}(x)\right) n_{p}(s, d x)$ (before hedging). The agent chooses a $\pi$ to partially hedge $Z$ and $\tilde{Z}$. The aggregate penalty after hedging is given by $g_{1}\left(s, Z_{s}-\pi_{s} \sigma_{s}\right)+\int_{\mathbb{R} \backslash\{0\}} g_{2}\left(s, x, \tilde{Z}_{s}(x)-\right.$ $\left.\pi_{s} \beta_{s}(x)\right) n_{p}(s, d x)$. Summarizing, when the agent chooses a $\pi \in U$, he faces a tradeoff between: 
(a) Benefitting from the excess return $\pi_{s} b_{s}$.

(b) Diminishing the variability of the evaluation due to the locally Gaussian part. (That is, choosing $\pi$ such that $\left|Z_{s}-\pi_{s} \sigma_{s}\right|$ is small.)

(c) Diminishing the variability of the evaluation due to jumps. (That is, choosing $\pi$ such that $\left|\tilde{Z}_{s}-\pi_{s} \beta_{s}\right|$ is small.)

Note that (4.5) is a finite dimensional convex optimization problem that can be computed numerically efficiently; see the examples in Section 6 below. Since the portfolio choice problem is translation invariant, it is straightforward to see that the indifference valuation is given by $V_{0}(F)-V_{0}(0)$.

\subsection{Exponential Utility Under Multiple Priors Preferences}

A utility function that is particularly popular in insurance and financial mathematics (Goovaerts et al. [32], Föllmer and Schied [27] and Mania and Schweizer [53]) and decision theory (Gollier [31]) is the exponential utility function. When $u$ is exponential, we provide a solution to the portfolio choice and indifference valuation problems in the case that the penalty function $c$ in (2.3) is an indicator function. This means that we are in the multiple priors setting of Gilboa and Schmeidler [30]. Specifically, let $\gamma>0$ and consider the robust expected utility optimization problem

$$
\begin{aligned}
& V_{t}(F)=\operatorname{ess}_{\sup } \operatorname{su\mathcal {A}}_{t} U_{t}\left(F+X_{T}^{(\pi)}\right) \\
& :=\operatorname{ess}_{\sup } \operatorname{sess}_{\boldsymbol{\mathcal { A }}} \operatorname{esnf}_{Q \in M}-\left(\mathrm{E}_{Q}\left[\exp \left\{-\frac{F+X_{T}^{(\pi)}}{\gamma}\right\} \mid \mathcal{F}_{t}\right]\right) \text {, }
\end{aligned}
$$

for a weakly compact set $M \subset \mathcal{Q}$, further specified below. As $\exp \{-x\} \geq 0$, the expectation is well-defined for every trading strategy.

Contrary to Section 4.1, in which the economic agent is ambiguity averse but not risk averse (linear utility), the economic agent solving (4.7) is both ambiguity averse and risk averse. Note that $\gamma>0$ measures the absolute risk tolerance of the agent (with large values of $\gamma$ corresponding to a low level of risk aversion and low values corresponding to a high level of risk aversion). Subsequently, we will assume that $M$ has the form

$$
M=\left\{Q \in \mathcal{Q} \mid q_{s} \in C_{s}, \quad \psi_{s}(x) \in D_{s}(x) \text { for every } s \in[0, T], \quad x \in \mathbb{R}\right\},
$$

for convex, closed, bounded set-valued predictable mappings $C$ and $D$, given by $D:=\left\{\psi \mid d^{-}(x) \leq\right.$ $\left.\psi_{s}(x) \leq d_{s}^{+}(x)\right\}$ for bounded $L^{2, \infty}$ functions $-1+\epsilon \leq d_{s}^{-}(x) \leq 0 \leq d_{s}^{+}(x)$. The case that $C=\{0\}$ and $D=\{0\}$ corresponds to ambiguity neutrality, i.e., to effectively not considering any alternative probabilistic model at all; in this case, $M=\{P\}$ would hold.

For the set $M$ thus specified, define

$$
\begin{aligned}
\bar{g}_{1}(t, z): & =\sup _{q \in C_{t}} z q \\
\bar{g}_{2}(t, x, \tilde{z}): & =\gamma\left(\exp \left\{\frac{\tilde{z}(x)}{\gamma}\right\}-1\right)\left(d_{t}^{+}(x) I_{\{\tilde{z}(x) \geq 0\}}+d_{t}^{-}(x) I_{\{\tilde{z}(x)<0\}}\right) .
\end{aligned}
$$

If $C=\emptyset$, we set $\bar{g}_{1}:=0$. The next theorem shows that the solution to the optimization problem (4.7) can be obtained directly from the solution to a BSDE: 
Theorem 4.3 The solution to (4.7) is given by $V_{0}(F)=-\exp \left\{-\frac{1}{\gamma}\left(w_{0}+Y_{0}\right)\right\}$, where $Y_{t}$ is the unique solution to the BSDE with terminal condition $F$ and driver function:

$$
\begin{aligned}
f(s, z, \tilde{z}):=\inf _{\pi \in U}\{ & -\pi b_{s}+\frac{1}{2 \gamma}\left|z-\pi \sigma_{s}\right|^{2}+\bar{g}_{1}\left(s, z-\pi \sigma_{s}\right)+\int_{\mathbb{R} \backslash\{0\}} \bar{g}_{2}\left(s, x, \tilde{z}(x)-\pi \beta_{s}(x)\right) n_{p}(s, d x) \\
& \left.+\gamma \int_{\mathbb{R} \backslash\{0\}}\left(\exp \left\{\frac{\tilde{z}(x)-\pi \beta_{s}(x)}{\gamma}\right\}-1-\frac{\tilde{z}(x)-\pi \beta_{s}(x)}{\gamma}\right) n_{p}(s, d x)\right\}
\end{aligned}
$$

with $\bar{g}_{i}$ defined in (4.8) for $i=1,2$. Furthermore, the optimal strategy $\pi_{s}^{*}$ is a predictable process that attains the infimum in (4.9) for $(z, \tilde{z})=\left(Z_{s}, \tilde{Z}_{s}\right)$.

Remark 4.4 BSDEs have been a rather popular tool to solve the utility maximization problem under exponential utility, in a wide variety of settings (the exponential utility maximization problem is connected to the popular minimal entropy martingale measure and to the Esscher density). In the case that there are no jumps, i.e., $n_{p}=0$, and there is no ambiguity, i.e., $d^{+}=d^{-}=\lambda=0$, our general solution above reduces to the solution obtained by $\mathrm{Hu}$, Imkeller and Müller [38]; see also El Karoui and Rouge [24]. These results have been generalized for continuous price processes to continuous and non-continuous filtrations, see for instance, Mania and Schweizer [53] and Becherer [4], in a purely risk-based setting. Recently, Morlais [58] generalized the results by Becherer [4] by allowing for infinite activity jumps in the asset price processes. However, none of these works allow for ambiguity, as opposed to our setting. In the case that there are no jumps but there is (Brownian) ambiguity (i.e., $n_{p}=d^{+}=d^{-}=0$ ), our general solution above reduces to the solution obtained by Müller [59].

In (4.7), the economic agent is 'penalized' on the one hand for the risk he faces (represented by the $\gamma$-exponential utility) and on the other hand for the ambiguity he encounters (represented by the sets $C$ and $D$ ). As a result, the penalty in (4.9) features four terms (the terms with plus sign):

(1.) The first term is due to the (local) risk coming from the Brownian motion. This term would equal zero if the agent is not risk averse (i.e., if $\gamma \uparrow \infty$ ) or if, after hedging, there is no locally Gaussian randomness affecting the evaluation (i.e., if $Z_{s}-\pi_{s} \sigma_{s}=0$ ).

(2.) The second term, $\bar{g}_{1}\left(s, Z_{s}-\pi_{s} \sigma_{s}\right)$, is due to the (local) model uncertainty about the Brownian motion. This term would equal zero if the agent is not ambiguity averse, if there is no model uncertainty about the distribution of the Gaussian part (i.e., if $C=\{0\}$ ), or if, after hedging, there is no locally Gaussian randomness affecting the evaluation.

(3.) The third term, $\int_{\mathbb{R} \backslash\{0\}} \bar{g}_{2}\left(s, x, \tilde{Z}_{s}(x)-\pi_{s} \beta_{s}(x)\right) n_{p}(s, d x)$, is due to the (local) model uncertainty about the jumps. It would equal zero if the agent is not ambiguity averse, if there is no model uncertainty about the distribution of the jump part (i.e., $D=\{0\}$ and $d^{+}=d^{-}=0$ ) or if, after hedging, there is no randomness due to the jump part affecting the evaluation (i.e., if $\tilde{Z}_{s}-\pi_{s} \beta_{s}=0$ ).

(4.) The fourth term is due to the (local) risk coming from the jump part; it is the jump analog of (1.).

Note that $C$, the set of alternative models for the drift $q$, is uniformly bounded. Consequently, we must have that $\left|\bar{g}_{1}(s, z)\right| \leq$ const $|z|$. Therefore, in (4.9), the economic agent is 
penalized quadratically, by $\left|Z_{s}-\pi_{s} \sigma_{s}\right|^{2}$, due to the (local) risk coming from the Brownian motion, and linearly by a penalty bounded by $\left|Z_{s}-\pi_{s} \sigma_{s}\right|$, due to the (local) model uncertainty about the Brownian motion. Consequently, if $\left|Z_{s}-\pi_{s} \sigma_{s}\right|$ is small, i.e., if there is 'little' Brownian randomness left after hedging, then the penalty due to ambiguity will be larger than the penalty due to risk. From a Taylor expansion of the third and fourth terms in (4.9), it may be seen that the same observation is true for the jump part. Therefore, if there is only 'little' randomness left after hedging, the evaluation is more (negatively) affected by ambiguity than by risk. On the other hand, if there is 'much' Brownian randomness left after hedging, (meaning that $\left|Z_{s}-\pi_{s} \sigma_{s}\right|$ is large), then the penalty due to risk is of a higher order than the penalty due to ambiguity. It is interesting to note, however, that the latter effect is not true for the jump part, since the penalties for risk and ambiguity arising from the jump part are of the same order if $\left|\tilde{Z}_{s}-\pi_{s} \beta_{s}\right|$ is large. Since the problem is again translation invariant, it is straightforward to see that the indifference valuation is given by $V_{0}(F)-V_{0}(0)$.

\subsection{Logarithmic Utility Under Variational Preferences}

We now consider predictable trading strategies $\rho$ that represent the part of wealth (rather than the absolute amount) invested in stock $i$. The admissible trading strategies are supposed to take values in a compact set $\tilde{C} \subset \mathbb{R}^{1 \times n}$. We assume that $\tilde{C} \beta \in[-1+\delta, \infty)$ for a $\delta>0$. We denote the set of all admissible trading strategies by $\mathcal{A}$; it is the set of all $\mathbb{R}^{1 \times n}$-valued predictable processes $\rho$ with $\rho_{t} \in \tilde{C}, d P \times d t$ a.s. The wealth process $X^{(\rho)}$ of a trading strategy $\rho$ with initial capital $w_{0}$ satisfies

$$
\begin{aligned}
X_{t}^{(\rho)} & =w_{0}+\sum_{i=1}^{n} \int_{0}^{t} X_{u-}^{(\rho)} \frac{\rho_{u}^{i}}{S_{u-}^{i}} d S_{u}^{i} \\
& =w_{0}+\int_{0}^{t} X_{u-}^{(\rho)} \rho_{u}\left(\sigma_{u} d W_{u}+b_{u} d u\right)+\int_{0}^{t} \int_{\mathbb{R} \backslash\{0\}} X_{u-}^{(\rho)} \rho_{u} \beta_{u}(x) \tilde{N}_{p}(d x, d u) .
\end{aligned}
$$

It follows that

$$
X_{t}^{(\rho)}=w_{0} \mathcal{E}\left(\int_{0}^{t} \rho_{u} \sigma_{u} d W_{u}+\int_{0}^{t} \rho_{u} b_{u} d u+\int_{0}^{t} \int_{\mathbb{R} \backslash\{0\}} \rho_{u} \beta_{u}(x) \tilde{N}_{p}(d u, d x)\right) .
$$

We solve the optimization problem

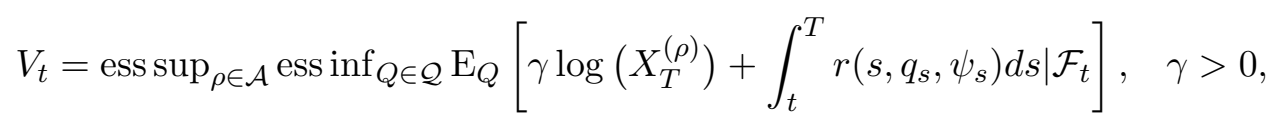

with $r\left(s, q_{s}, \psi_{s}\right)=r_{1}\left(t, q_{s}\right)+\int_{\mathbb{R} \backslash\{0\}} r_{2}\left(s, x, \psi_{s}(x)\right) n_{p}(s, d x)$, satisfying (H1)-(H3). Let

$$
\begin{aligned}
f(s, z, \tilde{z}):= & \inf _{\rho \in C}\left\{-\gamma \rho b_{s}-\gamma \int_{\mathbb{R} \backslash\{0\}}\left[\log \left(1+\rho \beta_{s}(x)\right)-\rho \beta_{s}(x)\right] n_{p}(s, d x)\right. \\
& \left.+\frac{\gamma}{2}\left|\rho \sigma_{s}\right|^{2}+g_{1}\left(s, z-\gamma \rho \sigma_{s}\right)+\int_{\mathbb{R} \backslash\{0\}} g_{2}\left(s, x, \tilde{z}(x)-\gamma \log \left(1+\rho \beta_{s}(x)\right)\right) n_{p}(s, d x)\right\} .
\end{aligned}
$$

We consider the BSDE

$$
Y_{t}=0+\int_{t}^{T} f\left(s, Z_{s}, \tilde{Z}_{s}\right) d s-\int_{t}^{T} Z_{s} d W_{s}-\int_{t}^{T} \tilde{Z}_{s}(x) \tilde{N}_{p}(d s, d x), \quad t \in[0, T] .
$$


Theorem 4.5 The BSDE (4.13) has a unique solution $(Y, Z, \tilde{Z})$ and the solution to (4.11) is given by

$$
V_{0}=Y_{0}+\gamma \log \left(w_{0}\right) .
$$

Furthermore, the optimal strategy $\rho_{s}^{*}$ is a predictable process that attains the infimum in (4.12) for $(z, \tilde{z})=\left(Z_{s}, \tilde{Z}_{s}\right)$.

Remark 4.6 In the case that there are no jumps and there is no ambiguity, problem (4.11) is solved by $\mathrm{Hu}$, Imkeller and Müller [38]. The case of ambiguity without jumps is considered by Müller [59]. For the case of a degenerate jump distribution with a penalty function $c$ given by the relative entropy, see Jeanblanc, Matoussi and Ngoupeyou [41]. These results all occur as special cases of our general solution provided above.

\section{Decomposition of The Excess Return}

Let us consider the case in which the trading set $U$ is specified as $\left[u_{\text {lower }}^{1}, u_{\text {upper }}^{1}\right] \times \ldots \times$ $\left[u_{\text {lower }}^{n}, u_{\text {upper }}^{n}\right]$, for $-\infty<u_{\text {lower }}^{i} \leq u_{\text {upper }}^{i}<\infty, i=1, \ldots, n$. That is, the economic agent is allowed to buy (shortsell) at most an amount of $u_{\text {upper }}^{i}\left(u_{\text {lower }}^{i}\right)$ of stock $i$. Suppose first that $u$ is linear. We then have that

$$
\begin{aligned}
f(s, z, \tilde{z})=\inf _{u_{\text {lower }} \leq \pi \leq u_{\text {upper }}}\{- & \pi b_{s}+g_{1}\left(s, z-\pi \sigma_{s}\right) \\
& \left.+\int_{\mathbb{R} \backslash\{0\}} g_{2}\left(s, x, \tilde{z}(x)-\pi \beta_{s}(x)\right) n_{p}(s, d x)\right\} .
\end{aligned}
$$

The function $f$ consists of a penalty (the two terms with plus sign) minus a reward. Denote by $\pi_{s}^{*}$ the optimal strategy attaining the infimum in (5.1). For the sake of simplicity, let us assume that $g_{1}$ and $g_{2}$ are differentiable in their last component. For a bounded square-integrable $\tilde{z}$, the Karush-Kuhn-Tucker conditions (see Theorem 28.3 in [64]) then imply that attaining the infimum in (5.1) is equivalent to the existence of Lagrange multipliers $0 \leq \mu_{s}^{*}, \zeta_{s}^{*} \in \mathbb{R}^{n}$ such that

$$
0=\mu_{s}^{*}-\zeta_{s}^{*}-b_{s}-\sigma_{s} \partial g_{1}\left(s, z-\pi_{s}^{*} \sigma_{s}\right)-\int_{\mathbb{R} \backslash\{0\}} \partial g_{2}\left(s, x, \tilde{z}(x)-\pi_{s}^{*} \beta_{s}(x)\right) \beta_{s}(x) n_{p}(s, d x),
$$

where the integral is understood componentwise. Furthermore, $\mu_{s}^{*}, \zeta_{s}^{*}$ satisfy the complimentary conditions, i.e.,

$$
\mu_{s}^{*} \cdot\left(\pi_{s}^{*} \sigma_{s}-u_{\text {upper }}\right)^{\top}=0 \text { and } \zeta_{s}^{*} \cdot\left(\pi_{s}^{*} \sigma_{s}-u_{\text {lower }}\right)^{\top}=0,
$$

where $u_{\text {upper }}\left(u_{\text {lower }}\right)$ denotes the vector consisting of the components $u_{\text {upper }}^{i}\left(u_{\text {lower }}^{i}\right)$ and . denotes componentwise multiplication. Notice that we interchanged integration and differentiation, which is permitted by the results on the subdifferential in Lemma A.7 in the Appendix.

Note that (5.2)-(5.3) is a convex optimization problem, which can be computed numerically efficiently. In particular, (5.2) yields that the excess return must satisfy

$$
\begin{aligned}
b_{s}=\left(\mu_{s}^{*}-\zeta_{s}^{*}\right)-\sigma_{s} \partial g_{1}\left(s, Z_{s}-\pi_{s}^{*} \sigma_{s}\right) & \\
& -\int_{\mathbb{R} \backslash\{0\}} \partial g_{2}\left(s, x, \tilde{Z}_{s}(x)-\pi_{s}^{*} \beta_{s}(x)\right) \beta_{s}(x) n_{p}(s, d x) .
\end{aligned}
$$


Hence, under linear utility, the excess return can be decomposed into three parts: The first term on the right-hand side of (5.4) is due to the trading constraints, the second term is an ambiguity premium due to model uncertainty about the Brownian motion, and the third term is an ambiguity premium due to model uncertainty about the jumps. Note that the Lagrange multiplier $\mu_{s}^{*}$ and $\zeta_{s}^{*}$ represent the sensitivity of $f$, the difference between penalty and reward, with respect to the upper and lower hedging constraints. Furthermore, $\sigma_{s} \partial g_{1}\left(s, Z_{s}-\pi_{s}^{*} \sigma_{s}\right)$ may be seen as the sensitivity with respect to the penalty arising from the locally Gaussian part. Finally, $\int_{\mathbb{R} \backslash\{0\}} \partial g_{2}\left(s, x, \tilde{Z}_{s}(x)-\pi_{s}^{*} \beta_{s}(x)\right) \beta_{s}(x) n_{p}(s, d x)$ may be seen as the sensitivity of the penalty arising from the jumps. Hence, (5.4) yields that the excess return is the sum of the agent's different sensitivities with respect to the constraints and to the two sources of ambiguity.

Next, let us look at the case of an exponential utility function as considered in Section 4.2, so that the economic agent is not only ambiguity averse but also risk averse. Using a similar argument as above, it may be seen that we obtain the following decomposition of the excess return:

$$
\begin{aligned}
b_{s}= & \left(\mu_{s}^{*}-\zeta_{s}^{*}\right)-\frac{\sigma_{s} Z_{s}^{\top}-\sigma_{s} \sigma_{s}^{\top} \pi_{s}^{*}}{\gamma}-\sigma_{s} \partial \bar{g}_{1}\left(s, Z_{s}-\pi_{s}^{*} \sigma_{s}\right) \\
& -\int_{\mathbb{R} \backslash\{0\}} \partial \bar{g}_{2}\left(s, x, \tilde{Z}_{s}(x)-\pi_{s}^{*} \beta_{s}(x)\right) \beta_{s}(x) n_{p}(s, d x) \\
& -\int_{\mathbb{R} \backslash\{0\}}\left(\exp \left\{\frac{\tilde{Z}_{s}(x)-\pi_{s}^{*} \beta_{s}(x)}{\gamma}\right\}-1\right) \beta_{s}(x) n_{p}(s, d x),
\end{aligned}
$$

where equality holds with respect to elements in the subgradients. Note that the first term on the right-hand side of (5.5) is again due to the trading constraints. The second term is a risk premium due to the risk arising from the Brownian motion, and the third term is an ambiguity premium due to model uncertainty about the Brownian motion. Furthermore, the fourth term is an ambiguity premium due to model uncertainty about the jumps, and the fifth term is a risk premium due to the risk arising from the jump part.

In the case of a logarithmic utility as considered in Section 4.3, we obtain

$$
\begin{aligned}
b_{s}= & \frac{\mu_{s}^{*}-\zeta_{s}^{*}}{\gamma}-\int_{\mathbb{R} \backslash\{0\}}\left[\frac{\beta_{s}(x)}{1+\rho^{*} \beta_{s}(x)}-\beta_{s}(x)\right] n_{p}(s, d x)+\sigma_{s} \sigma_{s}^{\top} \rho^{*} \\
& -\sigma_{s} \partial g_{1}\left(s, z-\gamma \rho^{*} \sigma_{s}\right) \\
& -\int_{\mathbb{R} \backslash\{0\}} \partial g_{2}\left(s, x, \tilde{z}(x)-\gamma \log \left(1+\rho^{*} \beta_{s}(x)\right) \frac{\beta_{s}(x)}{1+\rho^{*} \beta_{s}(x)} n_{p}(s, d x) .\right.
\end{aligned}
$$

The different terms arising can be interpreted similarly as above. Multiplying both sides in (5.4)-(5.6) by $\sigma_{s}^{\top}\left(\sigma_{s} \sigma_{s}^{\top}\right)^{-1}$, one obtains similar decompositions for the 'market price of uncertainty'.

\section{Numerical Implementation}

\subsection{Some Analytics}

Reconsider the setting of Section 5, with $d=1$. The generalization to higher dimensions is straightforward. Suppose further for simplicity that the jump component is time-homogeneous 
and features only finite activity jumps with degenerate jump size. In this case, we can integrate with respect to a Poisson process $d N_{t}$, instead of with respect to $\tilde{N}(d t, d x)$. Write $d \tilde{N}_{t}:=$ $d N_{t}-a d t$, where $a$ is the intensity of $N$ under $P$. Note that, for fixed $\omega$ and $t, \beta_{t}$ and $\tilde{Z}_{t}$ now correspond to real numbers and not to functions. We furthermore assume that $b, \sigma$, and $\beta$ do not depend on $\omega$ and $t$.

\subsubsection{Example 1:}

Let $p^{\prime} \geq 2$ and consider the penalty function

$$
r(t, q, \psi):=\alpha\left(\frac{1}{p^{\prime}}|q|^{p^{\prime}}+\{(1+\psi) \log (1+\psi)-\psi\} a\right), \quad \alpha>0 .
$$

For $p^{\prime}=2$, this penalty function corresponds to the relative entropy (3.3). For $p^{\prime}>2$, small values of the drift $q$, which induces (jointly with $\psi$ ) the alternative probabilistic model, are penalized less, while large values of $q$ are penalized more. Let $1<p \leq 2$ be such that $1 / p+1 / p^{\prime}=1$. Then, with linear utility, the corresponding driver $f$ in (5.1) reduces to

$$
\begin{aligned}
f(z, \tilde{z})=\inf _{u_{\text {lower }} \leq \pi \leq u_{\text {upper }}}\{ & -\pi b+\frac{1}{p \alpha}|z-\pi \sigma|^{p} \\
& \left.+\alpha\left(\exp \left\{-\frac{1}{\alpha}(\pi \beta-\tilde{z})\right\}-1+\frac{1}{\alpha}(\pi \beta-\tilde{z})\right) a\right\} .
\end{aligned}
$$

One easily verifies the following proposition:

Proposition 6.1 Let $h_{z, \tilde{z}}: \mathbb{R} \rightarrow \mathbb{R}$ be the function given by

$$
h_{z, \tilde{z}}(\pi)=-b+\frac{\sigma \operatorname{sign}(\pi \sigma-z)}{\alpha}|\pi \sigma-z|^{p-1}-\beta\left(\exp \left\{-\frac{1}{\alpha}(\pi \beta-\tilde{z})\right\}-1\right) a .
$$

The driver function $f$ in (6.1) is explicitly obtained by plugging $\pi^{*}:=\left(u_{\text {lower }} \vee h_{z, \tilde{z}}^{-1}(0)\right) \wedge u_{\text {upper }}$ into the right-hand side of (6.1), i.e., $\pi^{*}$ solves the optimization problem.

\subsubsection{Example 2:}

Next, let us look at the case of an exponential utility function and consider the set

$$
C=\left\{\left.q \in \mathbb{R}|| q\right|_{\infty} \leq \lambda\right\} \text {. Hence, } \bar{g}_{1}(z)=\lambda|z| \text {, for } \lambda \geq 0 .
$$

Furthermore, let $D=\left\{\psi \mid d^{-} \leq \psi \leq d^{+}\right\}$. The definition of $D$ implies that for the new intensity $a^{Q}$, we have $\left(1+d^{-}\right) a \leq a^{Q}=(1+\psi) a \leq\left(1+d^{+}\right) a$. Hence,

$$
\bar{g}_{2}(\pi, \tilde{z}):=\gamma \max \left(d^{+}\left(-1+\exp \left\{-\frac{1}{\gamma}(\pi \beta-\tilde{z})\right\}\right), d^{-}\left(-1+\exp \left\{-\frac{1}{\gamma}(\pi \beta-\tilde{z})\right\}\right)\right) .
$$

Robust exponential utility functionals are also considered in Laeven and Stadje [47]. Then the driver function corresponding to the optimal portfolio choice is given by (cf. (4.9))

$$
\begin{aligned}
f(z, \tilde{z})=\inf _{u_{\text {lower } \leq \pi \leq u_{\text {upper }}}\{}\{ & \pi b+\frac{1}{2 \gamma}|z-\pi \sigma|^{2}+\lambda|z-\pi \sigma| \\
& \left.+\left(\gamma\left(\exp \left\{-\frac{1}{\gamma}(\pi \beta-\tilde{z})\right\}-1+\frac{1}{\gamma}(\pi \beta-\tilde{z})\right)+\bar{g}_{2}(\pi, \tilde{z})\right) a\right\} .
\end{aligned}
$$


To solve minimization problem (6.2), we take the derivative with respect to $\pi$, set it equal to zero, and divide by $\sigma^{2}$. This yields

$$
\begin{aligned}
0 & \in-\frac{b}{\sigma^{2}}+\frac{1}{\gamma}\left(\pi^{*}-\frac{z}{\sigma}\right)-\frac{\lambda}{\sigma} \operatorname{sign}\left(\frac{z}{\sigma}-\pi^{*}\right)+\frac{\beta}{\sigma^{2}} a \\
& -\frac{\beta}{\sigma^{2}} a\left(1+d^{+} I_{\{\tilde{z}>\pi \beta\}}+d^{-} I_{\{\tilde{z}<\pi \beta\}}+I_{\{\tilde{z}=\pi \beta\}}\left[d^{-}, d^{+}\right]\right) \exp \left\{-\frac{\pi^{*} \beta-\tilde{z}}{\gamma}\right\}:=h_{z, \tilde{z}}\left(\pi^{*}\right) .
\end{aligned}
$$

One easily verifies the following proposition:

Proposition 6.2 The driver function $f$ in (6.2) is explicitly obtained by plugging $\pi^{*}:=$ $\left(u_{\text {lower }} \vee h_{z, \tilde{z}}^{-1}(0)\right) \wedge u_{\text {upper }}$ into the right-hand side of (6.2), i.e., $\pi^{*}$ solves the optimization problem.

We note that in this example $h_{z, \tilde{z}}^{-1}(0)$ can be computed, for instance, by using Newton's algorithm for every $(z, \tilde{z}) \in \mathbb{R}^{2}$.

\subsection{Algorithm}

In the simplified setting of this section, we can write (4.6) as

$$
Y_{t}=F-\int_{t}^{T} f\left(s, Z_{s}, \tilde{Z}_{s}\right) d s+\int_{t}^{T} Z_{s} d W_{s}+\int_{t}^{T} \tilde{Z}_{s} d \tilde{N}_{s}, \quad t \in[0, T] .
$$

Assume that $F=H\left(W_{T}, N_{T}\right)$ for a function $H: \mathbb{R}^{2} \rightarrow \mathbb{R}$. The discrete-time BS $\Delta \mathrm{E}$ corresponding to (6.3) is given by

$$
Y_{i h}=Y_{(i+1) h}-f\left(i h, Z_{i h}, \tilde{Z}_{i h}\right) h+Z_{i h} \Delta W_{(i+1) h}+\tilde{Z}_{i h} \Delta \tilde{N}_{(i+1) h}
$$

Taking conditional expectations on both sides, we obtain

$$
Y_{i h}=\mathrm{E}\left[Y_{(i+1) h} \mid \mathcal{F}_{i h}\right]-f\left(i h, Z_{i h}, \tilde{Z}_{i h}\right) h .
$$

We solve (6.4)-(6.5) by backward recursion, using a 'Longstaff-Schwartz type' of regression. For similar (yet slightly different) approaches in the case of a Brownian filtration, see Bender and Steiner [5] and the references therein.

Define an equi-spaced time grid $\{0, h, 2 h, 3 h, \ldots, T\}$ consisting of $L+1$ points with $T=L h$. Simulate $M$ paths of the Brownian motion $W$ and the Poisson process $N$, generating values $w_{i, k}$ and $n_{i, k}$, where $w_{i, k}\left(n_{i, k}\right)$ is the value of the $k$-th path of the Brownian motion (Poisson process) at time $i h$. Denote by $\Delta n_{(i+1), k}=n_{(i+1), k}-n_{i, k}$ and define $\Delta w_{(i+1), k}$ similarly. We aim to compute the corresponding $y_{i, k}$, and we know that, at maturity, $y_{T, k}=H\left(w_{T, k}, n_{T, k}\right)$. For this purpose, we first compute approximations to $\mathrm{E}\left[Y_{(i+1) h} \mid \mathcal{F}_{i h}\right], Z_{i h}$ and $\tilde{Z}_{i h}$, depending on the simulated paths. This proceeds in the following way. It follows from (6.4)-(6.5) that

$$
Y_{(i+1) h}-\mathrm{E}\left[Y_{(i+1) h} \mid \mathcal{F}_{i h}\right]=-Z_{i h} \Delta W_{(i+1) h}-\tilde{Z}_{i h} \Delta \tilde{N}_{(i+1) h}
$$

But this entails that we can obtain $\mathrm{E}\left[Y_{(i+1) h} \mid \mathcal{F}_{i h}\right],-Z_{i h}$ and $-\tilde{Z}_{i h}$ as the argmin of the minimization problem

$$
\min _{a_{i h}, b_{i h}, c_{i h}} \mathrm{E}\left[\left(Y_{(i+1) h}-a_{i h}-b_{i h} \Delta W_{(i+1) h}-c_{i h} \Delta \tilde{N}_{(i+1) h}\right)^{2} \mid \mathcal{F}_{i h}\right],
$$


with the minimum attained in $a_{i h}^{*}:=\mathrm{E}\left[Y_{(i+1) h} \mid \mathcal{F}_{i h}\right], b_{i h}^{*}:=-Z_{i h}$ and $c_{i h}^{*}:=-\tilde{Z}_{i h}$.

Since all the quantities involved are $\mathcal{F}_{i h}$ measurable and the problem is Markov, there exist functions $A, B, C: \mathbb{R}^{2} \rightarrow \mathbb{R}$ such that $A\left(W_{i h}, N_{i h}\right)=\mathrm{E}\left[Y_{(i+1) h} \mid \mathcal{F}_{i h}\right], B\left(W_{i h}, N_{i h}\right):=-Z_{i h}$ and $C\left(W_{i h}, N_{i h}\right):=-\tilde{Z}_{i h}$. We fix $K \in \mathbb{N}$ and assume that there exists constants $a_{j_{1}, j_{2}}, b_{j_{1}^{\prime}, j_{2}^{\prime}}, c_{\bar{j}_{1}, \bar{j}_{2}} \in$ $\mathbb{R}$ such that $\mathrm{E}\left[Y_{(i+1) h} \mid \mathcal{F}_{i h}\right] \approx \sum_{j_{1}=0, j_{2}=0}^{K} a_{j_{1}, j_{2}} W_{i h}^{j_{1}} N_{i h}^{j_{2}},-Z_{i h} \approx \sum_{j_{1}^{\prime}=0, j_{2}^{\prime}=0}^{K} b_{j_{1}^{\prime} j_{2}^{\prime}} W_{i h}^{j_{1}^{\prime}} N_{i h}^{j_{2}^{\prime}}$, and $-\tilde{Z}_{i h} \approx \sum_{\bar{j}_{1}=0, \bar{j}_{2}=0}^{K} c_{\bar{j}_{1}, \bar{j}_{2}} W_{i h}^{\bar{j}_{1}} N_{i h}^{\bar{j}_{2}}$. Then (6.6) suggests to calculate the desired approximations to $\mathrm{E}\left[Y_{(i+1) h} \mid \mathcal{F}_{i h}\right], Z_{i h}$ and $\tilde{Z}_{i h}$, given our simulated paths and $y_{i+1, k}$, using the following algorithm:

$$
\begin{aligned}
\min _{a_{j_{1}, j_{2},}, b_{j_{1}^{\prime}, j_{2}^{\prime}, \bar{j}_{1}, \bar{j}_{2}}} \sum_{k=0}^{M}\left(y_{(i+1), k}\right. & -\sum_{j_{1}=0, j_{2}=0}^{K} a_{j_{1}, j_{2}} w_{i, k}^{j_{1}} n_{i, k}^{j_{2}}-\sum_{j_{1}^{\prime}=0, j_{2}^{\prime}=0}^{K} b_{j_{1}^{\prime}, j_{2}^{\prime}}\left[w_{i, k}^{j_{1}^{\prime}} n_{i, k}^{j_{2}^{\prime}} \Delta w_{i+1, k}\right] \\
& \left.-\sum_{\bar{j}_{1}=0, \bar{j}_{2}=0}^{K} c_{\bar{j}_{1}, \bar{j}_{2}}\left[w_{i, k}^{\bar{j}_{1}} n_{i, k}^{\bar{j}_{2}}\left(I_{\left\{\Delta n_{(i+1), k}=0\right\}}-a h\right)\right]\right)^{2} .
\end{aligned}
$$

Note that this is a linear least squares regression in the $3(K+1)^{2}$ constants $a_{j_{1}, j_{2}}, b_{j_{1}^{\prime}, j_{2}^{\prime}}, c_{\bar{j}_{1}, \bar{j}_{2}}$. (Of course, other choices of basis functions and other types of regressions are also possible.)

Denote the constants that attain the minimum in (6.7) by $a^{*}, b^{*}, c^{*}$ and set $\mathrm{E}\left[Y_{(i+1) h} \mid \mathcal{F}_{i h}\right] \approx$ $\sum_{j_{1}=0, j_{2}=0}^{K} a_{j_{1}, j_{2}}^{*} w_{i, k}^{j_{1}} n_{i, k}^{j_{2}}, Z_{i h} \approx-\sum_{j_{1}^{\prime}=0, j_{2}^{\prime}=0}^{K} b_{j_{1}^{\prime}, j_{2}^{\prime}}^{*} w_{i, k}^{j_{1}^{\prime}} n_{i, k}^{j_{2}^{\prime}}$, and $\tilde{Z}_{i h} \approx-\sum_{\bar{j}_{1}=0, \bar{j}_{2}=0}^{K} c_{\bar{j}_{1}, \bar{j}_{2}}^{*} w_{i, k}^{\bar{j}_{1}} n_{i, k}^{\bar{j}_{2}}$. Finally, by (6.5), one can then calculate $y_{i, k}$ by

$$
y_{i, k}=\sum_{j_{1}=0, j_{2}=0}^{K} a_{j_{1}, j_{2}}^{*} w_{i, k}^{j_{1}} n_{i, k}^{j_{2}}-f\left(i h,-\sum_{j_{1}^{\prime}=0, j_{2}^{\prime}=0}^{K} b_{j_{1}^{\prime}, j_{2}^{\prime}}^{*} w_{i, k}^{j_{1}^{\prime}} n_{i, k}^{j_{2}^{\prime}},-\sum_{\bar{j}_{1}=0, \bar{j}_{2}=0}^{K} c_{\bar{j}_{1}, \bar{j}_{2}}^{*} w_{i, k}^{\bar{j}_{1}} n_{i, k}^{\bar{j}_{2}}\right) h .
$$

\subsection{Some Numerical Results}

We show below numerical results for various special cases of Example 2 in Subsection 6.1.2. We consider a European put option with strike price 2 and time-to-maturity of 0.5 years. We take $b=0.04, \sigma=0.2, a=1, \beta=0.03, u_{\text {upper }}=10$ and $u_{\text {lower }}=0$. The number of simulations is 10,000. Figure 1 plots $Y_{0}$ as a function of $\gamma$. We consider subsequently the case of (i) no ambiguity $\left(\lambda=d_{+}=d_{-}=0\right)$, no hedge; (ii) no ambiguity $\left(\lambda=d_{+}=d_{-}=0\right.$ ), with hedge; (iii) Brownian ambiguity only $\left(\lambda=0.05, d_{+}=d_{-}=0\right)$, with hedge; (iv) jump ambiguity only $\left(\lambda=0, d_{+}=0.5, d_{-}=-0.25\right)$, with hedge; (v) both Brownian ambiguity and jump ambiguity $\left(\lambda=0.05, d_{+}=0.5, d_{-}=-0.25\right)$, with hedge. In the limit, as $\gamma$ tends to infinity, the risk-averse $\gamma$-exponential utility maximizer becomes risk-neutral: (vi) no ambiguity $\left(\lambda=d_{+}=d_{-}=0\right.$ ), risk neutrality (asymptote $\gamma=\infty$ ), no hedge; (vii) no ambiguity $\left(\lambda=d_{+}=d_{-}=0\right.$ ), risk neutrality (asymptote $\gamma=\infty$ ), with hedge. The figure shows clearly that risk aversion and ambiguity aversion decrease the evaluation, and that hedging opportunities increase the evaluation. 


\section{A Appendix}

\section{A.1 Preliminaries}

Let $k \in \mathbb{N}$ and define $\mathcal{X}:=\mathbb{R}^{k}$. Denote by $\mathcal{X}^{*}$ the topological dual of $\mathcal{X}$, which can be identified with $\mathbb{R}^{k}$. For a convex function $f: \mathcal{X} \rightarrow \mathbb{R} \cup\{\infty\}$, we define its subgradient as $\partial f(x)=\left\{x^{*} \in \mathcal{X}^{*} \mid f(y)-f(x) \geq x^{*}(y)-x^{*}(x)\right.$ for all $\left.y \in \mathcal{X}\right\}$. Since $x^{*}(x)$ can be identified with the scalar product of vectors $x^{*}$ and $x$, we sometimes write $x^{*} x$. If the function has several arguments, then the subdifferential should be taken with respect to the components in which the function is convex. For example, if $f(t, x)$ is convex in $x$, then we define $\partial f(t, x)=$ $\left\{x^{*} \in \mathcal{X}^{*} \mid f(t, y)-f(t, x) \geq x^{*}(y-x)\right.$ for all $\left.y \in \mathcal{X}\right\}$. For a convex function $f: \mathcal{X} \rightarrow \mathbb{R} \cup\{\infty\}$ not identical infinity, we denote by $f^{*}\left(x^{*}\right)=\sup _{x \in \mathcal{X}}\left\{x^{*}(x)-f(x)\right\}$ the dual conjugate of $f$ mapping from $\mathcal{X}^{*}$ to $\mathbb{R} \cup\{\infty\}$. Again, if the function has several arguments, the dual conjugate should be taken with respect to the components in which the function is convex. The next result can be found in Rockafellar [64], Theorem 23.5.

Proposition A.1 Let $f: \mathcal{X} \rightarrow \mathbb{R} \cup\{\infty\}$ be a convex and lower-semicontinuous function. Then for every $x_{0}^{*} \in \mathcal{X}^{*}$ and $x_{0} \in \mathcal{X}$, the following statements are equivalent:

(i) $x_{0} \in \partial f^{*}\left(x_{0}^{*}\right)$;

(ii) $x_{0}^{*} \in \partial f\left(x_{0}\right)$;

(iii) $f\left(x_{0}\right)=\max _{x^{*} \in \mathcal{X}^{*}}\left\{x^{*}\left(x_{0}\right)-f^{*}\left(x^{*}\right)\right\}=x_{0}^{*}\left(x_{0}\right)-f^{*}\left(x_{0}^{*}\right)$;

(iv) $f^{*}\left(x_{0}^{*}\right)=\max _{x \in \mathcal{X}}\left\{x_{0}^{*}(x)-f(x)\right\}=x_{0}^{*}\left(x_{0}\right)-f\left(x_{0}\right)$.

Let us recall some definitions:

Definition A.2 A predictable process $H:[0, T] \times \Omega \rightarrow \mathbb{R}$ is called locally integrable if $\int_{0}^{T}\left|H_{s}\right|^{2} d s$ $<\infty$ a.s. $A \mathcal{P} \otimes \mathbb{B}(\mathbb{R} \backslash\{0\})$-measurable function $\tilde{H}:[0, T] \times \Omega \times \mathbb{R} \backslash\{0\} \rightarrow \mathbb{R}$ is called locally integrable if $\int_{0}^{T} \int_{[-1,1] \backslash\{0\}}\left|\tilde{H}_{s}(x)\right|^{2} n_{p}(s, d x) d s<\infty$ and $\int_{0}^{T} \int_{\mathbb{R} \backslash[-1,1]}\left|\tilde{H}_{s}(x)\right| n_{p}(s, d x) d s<\infty$ a.s.

Definition A.3 We call a martingale $M$ a $B M O(P)$ if there exists a constant $c>0$ such that

$$
\mathrm{E}\left[\langle M\rangle_{T}-\langle M\rangle_{\sigma} \mid \mathcal{F}_{\sigma}\right] \leq c, \quad\left|\Delta M_{\sigma}\right|^{2} \leq c \text { for all stopping times } \sigma .
$$

Furthermore, we call $Z:[0, T] \times \Omega \rightarrow \mathbb{R}$ a $B M O(P)$ process if $Z$ is predictable and there exists a constant $C>0$ such that for every stopping time $\sigma$ we have $\mathrm{E}\left[\int_{\sigma}^{T}\left|Z_{s}\right|^{2} d s \mid \mathcal{F}_{\sigma}\right] \leq C$. We call $\tilde{Z}:[0, T] \times \Omega \times \mathbb{R} \backslash\{0\} \rightarrow \mathbb{R}$ a $B M O(P)$ function if $\tilde{Z}$ is $\mathcal{P} \otimes \mathcal{B}(\mathbb{R} \backslash\{0\})$-measurable, bounded, and there exists a constant $C>0$ such that for every stopping time $\sigma$ we have $\mathrm{E}\left[\int_{\sigma}^{T} \int_{\mathbb{R} \backslash\{0\}}\left|\tilde{Z}_{s}(x)\right|^{2} n_{p}(s, d x) d s \mid \mathcal{F}_{\sigma}\right] \leq C$.

If $Z$ and $\tilde{Z}$ are in $L^{2}(d P \times d s)$ and $L^{2}\left(d P \times n_{p}(s, d x) \times d s\right)$, respectively, then $M_{t}=\int_{0}^{t} Z_{s} d W_{s}+$ $\int_{0}^{t} \int_{\mathbb{R} \backslash\{0\}} \tilde{Z}_{s}(x) \tilde{N}(s, d x)$ is a square-integrable martingale. Furthermore, if $Z$ is a $\operatorname{BMO}(P)$ processes and $\tilde{Z}$ is a $\operatorname{BMO}(P)$ function, then $M$ is a $\operatorname{BMO}(P)$ martingale. We need the following result, also known as Kazamaki's [44] criterion.

Theorem A.4 If $M$ is a $B M O(P)$ and there exists a $\delta>0$ such that $\Delta M>-1+\delta$ then the stochastic exponential of $M, \mathcal{E}\left(M_{t}\right)$, is a uniformly integrable martingale. Furthermore, $\mathcal{E}\left(M_{T}\right)>0$. 


\section{A.2 Proofs}

We define

$$
(q \cdot W)_{t}:=\int_{0}^{t} q_{s} d W_{s} \quad \text { and } \quad\left(\tilde{Z} \cdot \tilde{N}_{p}\right)_{t}:=\int_{0}^{t} \int_{\mathbb{R} \backslash\{0\}} \tilde{Z}_{s}(x) \tilde{N}_{p}(d s, d x) .
$$

We denote $\Phi(x):=\exp \{x\}-x-1 \geq 0$ for $x \in \mathbb{R}$, and $\Psi(x):=\Phi^{*}(x)=(1+x) \log (1+x)-x \geq 0$ for $x \geq-1$ and infinity else. The Fenchel dual inequality implies that for all $x, y \in \mathbb{R}$,

$$
x y \leq \Psi(x)+\Phi(y) .
$$

To prove Theorem 4.1 we need the following inequalities:

Lemma A.5 The following inequalities hold for all $C, \alpha, \lambda>0$ :

$$
\begin{aligned}
\exp \left\{\frac{x}{\alpha}\right\}-\frac{x}{\alpha}-1 & \leq \frac{x^{2}}{\alpha^{2}} \exp \left\{\frac{C}{\alpha}\right\}, \quad \text { for all } \quad x \in[-C, C] ; \\
x^{2} & \leq 2 \alpha^{2} e^{C / \alpha}\left[\exp \left\{\frac{x}{\alpha}\right\}-\frac{x}{\alpha}-1\right], \quad \text { for all } x \in[-C, C] ; \\
\left|e^{x / \lambda}-1\right| & \leq e^{C / \lambda} \frac{|x|}{\lambda}, \quad \text { for all } \quad x \in(-\infty, C] .
\end{aligned}
$$

Proof. As $e^{C / \alpha}>1$, the first inequality can be seen from the sum expansion of the LHS (lefthand side) for $\frac{|x|}{\alpha}<1$. For $\frac{|x|}{\alpha} \geq 1$, one easily verifies that already $\exp \{C / \alpha\}$ is an upper bound for the LHS in the first inequality. The second inequality for $x>0$ can also be seen from the sum expansion of the RHS (right-hand side). For $x<0$, one can compare the derivatives of the functions $f_{1}(x)=x^{2}$ and $f_{2}(x)=2 \alpha^{2} e^{C / \alpha}\left[\exp \left\{\frac{x}{\alpha}\right\}-\frac{x}{\alpha}-1\right]$. Then $f_{1}^{\prime}(x)=2 x$ and $f_{2}^{\prime}(x)=2 \alpha e^{C / \alpha}\left(\exp \left\{\frac{x}{\alpha}\right\}-1\right)$. Now $f_{1}^{\prime}(0)=0=f_{2}^{\prime}(0)$ and $f_{1}^{\prime}(-C)>f_{2}^{\prime}(-C)$. As $f_{2}^{\prime}$ is convex and $f_{1}^{\prime}$ is linear this entails that $f_{1}^{\prime}(x) \geq f_{2}^{\prime}(x)$ for all $x \in[-C, 0]$. Therefore, for $x \in[-C, 0]$, $f_{1}(x)=-\int_{x}^{0} f_{1}^{\prime}(y) d y \leq-\int_{x}^{0} f_{2}^{\prime}(y) d y=f_{2}(x)$. This shows (A.3) for $x \in[-C, 0]$. Finally, to see (A.4), define $\hat{f}_{1}(x):=\left|e^{x / \lambda}-1\right|$ and $\hat{f}_{2}(x):=e^{C / \lambda} \frac{|x|}{\lambda}$. Then $\hat{f}_{1}(0)=0=\hat{f}_{2}(0)$. Furthermore, for $x \in(0, C]$, we have $\hat{f}_{1}^{\prime}(x)=\frac{e^{x / \lambda}}{\lambda} \leq \frac{e^{C / \lambda}}{\lambda}=\hat{f}_{2}^{\prime}(x)$. For $x<0, \hat{f}_{1}^{\prime}(x)=-\frac{e^{x / \lambda}}{\lambda} \geq-\frac{e^{C / \lambda}}{\lambda}=\hat{f}_{2}^{\prime}(x)$. From these inequalities (A.4) follows.

Corollary A.6 Suppose that $\tilde{K}$ is in $L^{2, \infty}$ (see Section 2) and is bounded. Then

$$
\begin{aligned}
& \left\|\sup _{t} \int_{\mathbb{R} \backslash\{0\}} \Phi\left(\tilde{K}_{t}(x)\right) n_{p}(t, d x)\right\|_{\infty}<\infty \\
& \left\|\sup _{t} \int_{\mathbb{R} \backslash\{0\}} \Phi^{2}\left(\tilde{K}_{t}(x)\right) n_{p}(t, d x)\right\|_{\infty}<\infty .
\end{aligned}
$$

Proof. The first statement follows immediately from (A.2) and the definition of $L^{2, \infty}$. Furthermore, if $\tilde{K}$ is bounded by a constant, say $C$, then (A.2) and the fact that $\Phi(x) \geq 0$ yields

$$
\Phi^{2}\left(\tilde{K}_{t}(x)\right) \leq \tilde{K}_{t}^{4}(x) \exp \{2 C\} \leq \tilde{K}_{t}^{2}(x) C^{2} \exp \{2 C\} .
$$

As $\tilde{K} \in L^{2, \infty}$, the second statement follows.

For a driver function $g(t, z, \tilde{z})=g_{1}(t, z)+\int_{\mathbb{R} \backslash\{0\}} g_{2}(t, x, \tilde{z}(x)) n_{p}(t, d x)$ of a BSDE with jumps, the following properties play an important role, while proving Theorem 4.1: 
(a) $0 \leq g_{i}$ for $i=1,2$ and $g_{1}(t, 0)=g_{2}(t, x, 0)=0$ for all $t, x$.

(b) There exist $\bar{K}, K_{2}>0$ and a bounded $\tilde{A} \in L^{2, \infty}$ such that for all $t \in[0, T]$, a.s. all $\omega$, all $z \in \mathbb{R}^{1 \times d}$, and all $\mathbb{B}(\mathbb{R} \backslash\{0\})$-measurable $\tilde{z}: \mathbb{R} \backslash\{0\} \rightarrow \mathbb{R}$ we have

$$
g_{1}(t, \omega, z) \leq \bar{K}\left(1+|z|^{2}\right) \quad \text { and } \quad g_{2}(t, \omega, x, \tilde{z}(x)) \leq \tilde{A}_{t}(\omega, x)+K_{2}\left(\exp \left\{\frac{\tilde{z}(x)}{K_{2}}\right\}-\frac{\tilde{z}(x)}{K_{2}}-1\right) \text {. }
$$

(c) (i) For every $t$, we have that $g_{1}(t, \cdot)$ and $g_{2}(t, x, \cdot)$ are convex and continuous. Furthermore, $\partial g_{1}(t, \cdot)$ and $\partial g_{2}(t, x, \cdot)$ are always nonempty. We also write $\partial g_{1}(t, z)$ and $\partial g_{2}(t, x, y)$ where the subdifferential should be taken with respect to the last components, $z$ and $y$, respectively.

(ii) $g_{1}$ and $g_{2}$ have modifications such that for every $z, x, y$ the mappings $(t, \omega) \rightarrow$ $g_{2}(t, \omega, z)$ and $(t, \omega) \rightarrow g_{2}(t, \omega, x, y)$ are predictable.

(d) There exists $\bar{K}_{1}>0$ such that for every $t \in[0, T]$, a.s. all $\omega$, and all $z \in \mathbb{R}^{1 \times d}$ we have that

$$
|a| \leq \bar{K}_{1}(1+|z|), \text { for all } a \in \partial g_{1}(t, \omega, z) .
$$

(e) For every $C>0$, there exists a bounded function $\left(\tilde{H}_{s}(x)\right)$ in $L^{2, \infty}$ and $\bar{C}, \bar{K}_{2}, \epsilon>0$ such that for every $t \in[0, T]$, a.s. all $\omega$, all $x \in \mathbb{R}, y \in[-C, C]$, and $a \in \partial g_{2}(t, \omega, x, y)$ we have

$$
\begin{aligned}
|a| & \leq \tilde{H}_{t}(\omega, x)+\bar{K}_{2}|y|, \\
(-1+\epsilon) & \leq a \leq \bar{C} .
\end{aligned}
$$

We will see later that assumption (a) may be relaxed and assumption (b) may be replaced by

(b') For every $C>0$, there exists a bounded $\tilde{A} \in L^{2, \infty}$ and $K^{\prime \prime}>0$ such that for all $t$, a.s. all $\omega$, and all $x$,

$$
\begin{aligned}
g_{2}(t, \omega, x, a) & \leq \tilde{A}_{t}(\omega, x)+K^{\prime \prime} a^{2} \text { for all } a \in[-C, C] . \text { Furthermore } \\
g_{1}(t, \omega, z) & \leq \bar{K}\left(1+|z|^{2}\right) \text { for all } z \in \mathbb{R}^{1 \times d}
\end{aligned}
$$

Note that assumptions (b'), (d), and (e) are generalizations of Kobylanski's [46] quadratic growth conditions to a setting with infinite activity jumps.

Lemma A.7 Under the assumptions (H1)-(H3), $g_{1}$ and $g_{2}$ defined in (4.2)-(4.3) satisfy (a)(e).

Proof. (a): By (4.2), $g_{1}(t, 0)=-\inf _{q} r_{1}(t, q)=0$, where the last equality is satisfied by (H1). As $0 \in \partial r_{1}(t, 0)$, by Proposition A.1 we get that $0 \in \partial g_{1}(t, 0)$. In particular, the convex function $g_{1}$ has its global minimum in zero. It follows that $g_{1} \geq 0$. The proof for $g_{2}$ is similar.

(b): We write

$$
\begin{aligned}
g_{2}(t, \omega, x, \tilde{z}(x)) & =\sup _{y \in \mathbb{R}}\left\{\tilde{z}(x) y-r_{2}(t, \omega, x, y)\right\} \\
& \leq \tilde{A}_{t}(\omega, x)+K_{2} \sup _{y \in \mathbb{R}}\left\{\frac{\tilde{z}(x)}{K_{2}} y-(1+y) \log (1+y)+y\right\} \\
& =\tilde{A}_{t}(\omega, x)+K_{2}\left[\exp \left\{\frac{\tilde{z}(x)}{K_{2}}\right\}-\frac{\tilde{z}(x)}{K_{2}}-1\right],
\end{aligned}
$$


where we used (H2) in the inequality. The inequality for $g_{1}$ follows similarly.

(c)(i): Clearly, $g_{1}$ and $g_{2}$ are convex in their last arguments. By (b), $g_{1}$ and $g_{2}$ are realvalued functions. Since $g_{1}$ and $g_{2}$ are convex it is well-known that this implies that $g_{1}$ and $g_{2}$ are continuous and subdifferentiable.

(c)(ii): Let us show that the mappings $(s, \omega) \rightarrow g_{1}(s, \omega, z)$ and $(s, \omega) \rightarrow g_{2}(s, \omega, x, y)$ are predictable. We prove this by arguing that the supremum in (4.2)-(4.3) can be restricted to be taken over a countable set of predictable mappings. Let $M=\left\{(s, \omega, q) \mid r_{1}(s, \omega, q)<\infty\right\}$. Note that $M$ is a $\mathcal{P} \otimes \mathbb{B}(\mathbb{R} \backslash\{0\})$ measurable set. Enumerate $\mathbb{Q}^{d}$ with $\left\{q_{1}, q_{2}, \ldots\right\}$. Denote by $\operatorname{dist}\left(\cdot, M_{s, \omega}\right)$ the Euclidean distance to the closed set $M_{s, \omega}=\left\{q \in \mathbb{R}^{d} \mid r_{1}(s, \omega, q)<\infty\right\}$. For $j=1,2, \ldots$ define the mapping $f_{j}(s, \omega):=\operatorname{dist}\left(q_{j}, M_{s, \omega}\right)$. By a measurable selection theorem for every $j$ we may choose a predictable process $\hat{q}_{j}(s, \omega) \in M_{s, \omega}$ such that

$$
f_{j}(s, \omega)=\left|q_{j}-\hat{q}_{j}(s, \omega)\right| \text {. }
$$

We claim that for every $(s, \omega)$ the set $B_{s, \omega}:=\left\{\hat{q}_{1}(s, \omega), \hat{q}_{2}(s, \omega), \ldots\right\}$ is dense in $M_{s, \omega}$. To see this, let $q \in M_{s, \omega}$ and let $\epsilon>0$. As $\mathbb{Q}^{d}$ is dense in $\mathbb{R}^{d}$, there exists a $j \in \mathbb{N}$ such that the corresponding $q_{j}$ is in an $\epsilon$-environment of $q$. Therefore,

$$
\left|\hat{q}_{j}(s, \omega)-q\right| \leq\left|\hat{q}_{j}(s, \omega)-q_{j}\right|+\left|q_{j}-q\right| \leq 2 \epsilon,
$$

where we used in the last inequality that $\left|\hat{q}_{j}(s, \omega)-q_{j}\right| \leq\left|q-q_{j}\right| \leq \epsilon$ by the definition of $\hat{q}_{j}$ and the fact that $q \in M_{s, \omega}$. In particular, for every $(s, \omega)$ we have that $\left\{\hat{q}_{1}(s, \omega), \hat{q}_{2}(s, \omega), \ldots\right\}$ is dense in $M_{s, \omega}$. Next, we claim that

$$
g_{1}(s, \omega, z)=\sup _{j=1,2 \ldots}\left\{z q_{j}(s, \omega)-r_{1}\left(s, \omega, q_{j}(s, \omega)\right)\right\} .
$$

Clearly, by definition $g_{1}$ is larger than or equal to the RHS. However, since the subgradient of $g_{1}$ is nonempty, for fixed $(s, \omega, z)$, there exists $q^{*} \in \partial g_{1}(s, \omega, z)$ such that

$$
g_{1}(s, \omega, z)=z q^{*}-r_{1}\left(s, \omega, q^{*}\right),
$$

see Proposition A.1. Now the fact that $\left\{q_{1}(s, \omega), q_{2}(s, \omega), \ldots\right\}$ is a basis for the domain of $r_{1}(s, \omega, \cdot)$, and the assumption that $r_{1}$ is continuous on its domain entail that the RHS of (A.5) is larger than the RHS of (A.6). Therefore, indeed (A.5) holds. As $g_{1}$ by (A.5) is the supremum of predictable processes, $(s, \omega) \rightarrow g_{1}(s, \omega, z)$ can be identified with a predicable process for every $z$. Since, by (c)(i), $g_{1}$ is continuous in $z, g_{1}$ is determined by the $z \in \mathbb{Q}^{d}$. Thus, a modification can be chosen being predictable for all $z$. Similarly, the statement follows for $(s, \omega) \rightarrow g_{2}(s, \omega, x, y)$ with fixed $x, y \in \mathbb{R}$.

(d): By Proposition A.1, we have for $t, \omega$ and $z$ that $q \in \partial g_{1}(t, \omega, z)$ if and only if $z \in$ $\partial r_{1}(t, \omega, q)$. Therefore, (H3) yields $|z| \geq-\hat{K}_{1}+\hat{K}_{2}|q|$. Thus, indeed $|q| \leq \hat{K}_{1}+\frac{|z|}{\hat{K}_{2}}$.

(e): For $t, \omega, y \in[-C, C]$, and $x \in \mathbb{R}$ choose $a \in \mathbb{R}$ with $a \in \partial g_{2}(t, \omega, x, y)$. By Proposition A.1, we have then that $y \in \partial r_{2}(t, \omega, x, a)$. Therefore, (H3) yields

$$
|y| \geq-\tilde{K}_{t}(\omega, x)+\hat{K}_{4}|\log (1+a)| .
$$

Now as $\tilde{K}_{t}$ is uniformly bounded by a constant, say $\bar{C}$, we must have that for all $t \in[0, T]$, a.s. all $\omega, y \in[-C, C]$ and $x \in \mathbb{R}$ that the corresponding $a \in \partial g_{2}(t, \omega, x, y)$ is uniformly bounded, and bounded away uniformly from 0 . Furthermore, by (A.4) we get

$$
|a| \leq\left|\exp \left(\frac{|y|+\tilde{K}_{t}(\omega, x)}{\hat{K}_{4}}\right)-1\right| \leq e^{(C+\bar{C}) / \hat{K}_{4}} \frac{|y|+\tilde{K}_{t}(\omega, x)}{\hat{K}_{4}} .
$$


This shows (e).

Remark A.8 Property (b) entails that there exist $K^{\prime}, K_{2}>0$ such that for all $t \in[0, T]$, a.s. all $\omega$, all $z \in \mathbb{R}^{1 \times d}$ and $\mathbb{B}(\mathbb{R} \backslash\{0\})$-measurable $\tilde{z}: \mathbb{R} \backslash\{0\} \rightarrow \mathbb{R}$

$$
\begin{aligned}
g_{1}(t, \omega, z) & +\int_{\mathbb{R} \backslash\{0\}} g_{2}(t, \omega, x, \tilde{z}(x)) n_{p}(t, \omega, d x) \\
& \leq K^{\prime}\left(1+|z|^{2}\right)+K_{2} \int_{\mathbb{R} \backslash\{0\}}\left(\exp \left\{\frac{\tilde{z}(x)}{K_{2}}\right\}-\frac{\tilde{z}(x)}{K_{2}}-1\right) n_{p}(t, \omega, d x) .
\end{aligned}
$$

Remark A.9 If $g_{1}$ and $g_{2}$ are real-valued, and convex in their last component, then for their subgradients many of the 'normal' rules for differentiation apply, see Theorem 23.8 and Theorem 23.9 in Rockafellar [64].

The next lemma can be proved similarly as Lemma A.7.

Lemma A.10 Suppose that we start with functions $g_{1}(t, z)$ and $g_{2}(t, x, \tilde{z})$ satisfying $(c)$ and denote by $r_{1}$ and $r_{2}$ the corresponding dual conjugates, i.e., for $t \in[0, T]$ we set

$$
\begin{aligned}
r_{1}(t, q): & =\sup _{z \in \mathbb{R}^{1 \times d}}\left\{z q-g_{1}(t, z)\right\}, \text { for } q \in \mathbb{R}^{d} ; \\
r_{2}(t, x, a): & =\sup _{y \in \mathbb{R}}\left\{y a-g_{2}(t, x, y)\right\}, \text { for } x, a \in \mathbb{R} .
\end{aligned}
$$

Then property (b) implies that there exist constants $K_{1}, K_{2}, K_{3}>0$ such that for all $t$, a.s. all $\omega$, all $q$ and all $\mathbb{B}(\mathbb{R} \backslash\{0\})$-measurable $\psi: \mathbb{R} \backslash\{0\} \rightarrow[-1, \infty)$ we have

$$
\begin{aligned}
r_{1}(t, \omega, q)+ & \int_{\mathbb{R} \backslash\{0\}} r_{2}(t, \omega, x, \psi(x)) n_{p}(t, \omega, d x) \\
& \geq-K_{1}+K_{2}|q|^{2}+K_{3} \int_{\mathbb{R} \backslash\{0\}}[(1+\psi(x)) \log (1+\psi(x))-\psi(x)] n_{p}(t, \omega, d x) .
\end{aligned}
$$

From the definition of variational preferences in (2.3), with $u=\mathrm{id}$, and (H1) we get

$$
\begin{aligned}
U_{t}(F)=\operatorname{essinf}\left\{\mathrm{E}_{Q}[F\right. & +\int_{t}^{T}\left[r_{1}\left(s, q_{s}\right)\right. \\
& \left.\left.\left.+\int_{\mathbb{R} \backslash\{0\}} r_{2}\left(s, x, \psi_{s}(x)\right) n_{p}(s, d x)\right] d s \mid \mathcal{F}_{t}\right] \mid Q \ll P\right\},
\end{aligned}
$$

which is the object under consideration in Theorem 4.1. For a measure $Q \ll P$, let $D_{t}=$ $\mathrm{E}\left[\frac{d Q}{d P} \mid \mathcal{F}_{t}\right]$ and $\tau=\inf \left\{t \in[0, T] \mid D_{t}=0\right\} \wedge T$.

Lemma A.11 For $T>t \geq \tau$ we have that $D_{t}=0$. Furthermore, if $\tau^{*}=\inf \left\{t>0 \mid D_{t-}=\right.$ $0\} \wedge T$ then $\tau=\tau^{*}$.

Proof. From the martingale stopping theorem for $T>t$,

$$
\mathrm{E}\left[D_{t} I_{\{t \geq \tau\}}+D_{t} I_{\{t<\tau\}}\right]=\mathrm{E}\left[D_{t}\right]=1=\mathrm{E}\left[D_{t \wedge \tau}\right]=\mathrm{E}\left[D_{t} I_{\{t<\tau\}}\right] .
$$


Thus, $\mathrm{E}\left[D_{t} I_{\{t>\tau\}}\right]=0$. As $D$ is non-negative, the first part of the lemma follows. To see the second part note that the only possibility for $\tau \neq \tau^{*}$ is that, for fixed $\omega$, the left-hand limit of the process $D$ is zero at a time instance $t$, but $D$ jumps (upwards) so that $D_{t-}=0<D_{t}$. In other words, for the increasing sequence of stopping times $\tau_{m}:=\inf \left\{t>0 \mid D_{t} \in(1 / m, 0)\right\} \wedge(T-1 / m)$ we have that $D$ jumps at $\tau^{\prime}:=\lim _{m} \tau_{m}$. However, as $\tau^{\prime}>\tau_{m}, \tau^{\prime}$ is a predictable stopping time, see Ch. III in Protter [63]. As the jumps of $D$ are totally inaccessible, since they are induced by a (inhomogeneous) Poisson random measure, $\tau^{\prime}$ a.s. cannot coincide with a jump time.

The next two lemmas are the analogues of Lemma 2.1 and Proposition 2.1 in Delbaen, Hu and Bao [19]. They are proved there in a Brownian setting but the proofs also hold in our setting with obvious modifications:

Lemma A.12 Suppose that (H1) holds. Then for any stopping time $\sigma$ and $F \in L^{\infty}\left(\mathcal{F}_{T}\right)$,

$$
U_{\sigma}(F)=\operatorname{essinf}\left\{\mathrm{E}_{Q}\left[F+\int_{\sigma}^{\tau}\left[r_{1}\left(s, q_{s}\right)+\int_{\mathbb{R} \backslash\{0\}} r_{2}\left(s, x, \psi_{s}(x)\right) n_{p}(s, d x)\right] d s \mid \mathcal{F}_{\sigma}\right] \mid Q \sim P\right\},
$$

where $Q \sim P$ means that $Q$ and $P$ are equivalent in the sense that they share the same zero sets.

Lemma A.13 Suppose that (H1) holds. Then for any $F \in L^{\infty}\left(\mathcal{F}_{T}\right)$ the process $U_{t}(F)$ defined by (A.8) has the following properties:

(1) For all $Q \ll P$ we have that $U_{t}(F)+\int_{0}^{\tau \wedge t}\left[r_{1}\left(s, q_{s}\right)+\int_{\mathbb{R} \backslash\{0\}} r_{2}\left(s, x, \psi_{s}(x)\right) n_{p}(s, d x)\right] d s$ is a $Q$-submartingale.

(2) If there is a probability measure $Q \ll P$ with

$$
U_{0}(F)=\mathrm{E}_{Q}\left[F+\int_{0}^{\tau}\left[r_{1}\left(s, q_{s}\right)+\int_{\mathbb{R} \backslash\{0\}} r_{2}\left(s, x, \psi_{s}(x)\right) n_{p}(s, d x)\right] d s\right],
$$

then $U_{t}(F)+\int_{0}^{\tau \wedge t}\left[r_{1}\left(s, q_{s}\right)+\int_{\mathbb{R} \backslash\{0\}} r_{2}\left(s, x, \psi_{s}(x)\right) n_{p}(s, d x)\right] d s$ is a $Q$-martingale.

As $r_{i}$ are non-negative and $r_{1}(t, 0)=r_{2}(t, x, 0)=0$, clearly, for any $m \in \mathbb{R}$ and for any $t$, we have $U_{t}(m)=m$. Furthermore, $U$ is monotone in the sense that $F \leq G$ implies that $U_{t}(F) \leq U_{t}(G)$. Therefore, for any $F \in L^{\infty}\left(\mathcal{F}_{T}\right)$ we have that $\left|U_{t}(F)\right| \leq\|F\|_{\infty}$. Thus, we can apply the Doob-Meyer decomposition theorem to obtain that there exists a unique predictable increasing process $A_{t}$ with $A_{0}=0$ and a local martingale $M_{t}$ with $M_{0}=0$ such that

$$
U_{t}(F)=U_{0}(F)+A_{t}-M_{t} .
$$

For $k>0$, set $C_{k}=\left\{Q \ll P \mid \mathrm{E}_{Q}\left[\int_{0}^{T}\left[r_{1}\left(s, q_{s}\right)+\int_{\mathbb{R} \backslash\{0\}} r_{2}\left(s, x, \psi_{s}(x)\right) n_{p}(s, d x)\right] d s\right] \leq k\right\}$. Now (H2) entails that for every fixed $k$ there is a constant $\bar{C}>0$ such that for every $Q \in C_{k}$,

$$
\mathrm{E}_{Q}\left[\int_{0}^{T}\left(\left|q_{s}\right|^{2} d s+\int_{\mathbb{R} \backslash\{0\}} \Psi\left(\psi_{s}(x)\right) n_{p}(s, d x)\right) d s\right] \leq \bar{C},
$$

with $\Psi(x)=(1+x) \log (1+x)-x$ for $x \geq 1$. Denote $\tilde{N}_{p}^{Q}(d t, d x):=\tilde{N}_{p}(d t, d x)-(1+$ $\left.\psi_{t}(x)\right) n_{p}(t, d x) d t$. By Jacod and Shiryaev [40], Ch. 3, Th. 3.11 and Lemma 3.14 for bounded locally integrable functionals, integrals with respect to $\tilde{N}_{p}^{Q}(d t, d x)$ give rise to local martingales with respect to $Q$. The next lemma shows that the local martingale in (A.9) is in fact a $\operatorname{BMO}(P)$ martingale. It prepares Theorem A.21, which is a key step in the proof of Theorem 4.1. 
Lemma A.14 Assume that the process $J$ is a semi-martingale, bounded by a constant $\tilde{C}$, with Doob-Meyer decomposition $J=J_{0}+A-M$ and $A$ is increasing or decreasing. Then there exist a $B M O(P)$ process $Z$ and a $B M O(P)$ function $\tilde{Z}$ such that

$$
M_{t}=\int_{0}^{t} Z_{s} d W_{s}+\int_{0}^{t} \int_{\mathbb{R} \backslash\{0\}} \tilde{Z}_{s}(x) \tilde{N}_{p}(d s, d x) .
$$

Furthermore, for every $k \in \mathbb{N}$ and $Q \in C_{k}$, we have that $Z \in L^{2}(d Q \times d s)$ and $\tilde{Z} \in L^{2}(d Q \times$ $\left.n_{p}^{Q}(s, d x) \times d s\right)$.

Proof. We only prove the lemma for $A$ increasing. The case that $A$ is decreasing follows by considering $-J$. By the (local) martingale representation theorem, there exist predictable processes $Z$ and $\tilde{Z}$ such that $M_{t}=(Z \cdot W)_{t}+\left(\tilde{Z} \cdot \tilde{N}_{p}\right)_{t}$. Note that the jumps of $M$ are all totally inaccessible. Since $A$ is predictable, a jump of $M$ cannot coincide with a jump of $A$. As the jumps of $J$ are uniformly bounded (since $J$ is uniformly bounded), the jumps of $M$ must be uniformly bounded too. In particular, $M$ is locally square-integrable. This implies that we may choose $Z$ and $\tilde{Z}$ in (A.11) such that

$$
P\left[\int_{0}^{T}\left|Z_{s}\right|^{2} d s<\infty\right]=1 \quad \text { and } \quad P\left[\int_{0}^{T} \int_{\mathbb{R} \backslash\{0\}}\left|\tilde{Z}_{s}(x)\right|^{2} n_{p}(s, d x) d s<\infty\right]=1 .
$$

Since $M$ cannot have a jump greater than 2||$J \|_{S^{\infty}}$ we also have that $|\tilde{Z}| \leq 2 \tilde{C}$.

We first start our analysis for general $Q \in C_{k}$ with corresponding $q$ and $\psi$. (Note that this includes the case that $Q=P$, since then $q=\psi=0$.) For every $m \in \mathbb{N}$, define

$$
\sigma_{m}=\inf \left\{t \geq\left. 0\left|\int_{0}^{t} \int_{\mathbb{R} \backslash\{0\}}\right| Z_{s}\right|^{2} d s \geq m \text { and } \int_{0}^{t} \int_{\mathbb{R} \backslash\{0\}}\left|\tilde{Z}_{s}(x)\right|^{2} n_{p}(s, d x) d s \geq m\right\} .
$$

Then $\sigma_{m} \rightarrow \infty P$-a.s. and therefore also $Q$-a.s. as $m$ tends to infinity. Clearly, $\left(Z \cdot W^{Q}\right)_{t \wedge \sigma_{m}}$ is a square-integrable $Q$-martingale. That $\left(\tilde{Z} \cdot \tilde{N}^{Q}\right)_{t \wedge \sigma_{m}}$ is a square-integrable $Q$-martingale as 
well, may be seen from:

$$
\begin{aligned}
\mathrm{E}_{Q}[ & \left.\int_{0}^{T \wedge \sigma_{m}} \int_{\mathbb{R} \backslash\{0\}}\left|\tilde{Z}_{s}(x)\right|^{2} n_{p}^{Q}(s, d x) d s\right] \\
& =\mathrm{E}_{Q}\left[\int_{0}^{T \wedge \sigma_{m}} \int_{\mathbb{R} \backslash\{0\}}\left|\tilde{Z}_{s}(x)\right|^{2}\left(1+\psi_{s}(x)\right) n_{p}(s, d x) d s\right] \\
& \leq m+\mathrm{E}_{Q}\left[\int_{0}^{T \wedge \sigma_{m}} \int_{\mathbb{R} \backslash\{0\}}\left|\tilde{Z}_{s}(x)\right|^{2} \psi_{s}(x) n_{p}(s, d x) d s\right] \\
& \leq m+\mathrm{E}_{Q}\left[\int_{0}^{T \wedge \sigma_{m}} \int_{\mathbb{R} \backslash\{0\}}\left[\Phi\left(\left|\tilde{Z}_{s}(x)\right|^{2}\right)+\Psi\left(\psi_{s}(x)\right)\right] n_{p}(s, d x) d s\right] \\
& \leq m+\bar{C}+\mathrm{E}_{Q}\left[\int_{0}^{T \wedge \sigma_{m}} \int_{\mathbb{R} \backslash\{0\}} \Phi\left(\left|\tilde{Z}_{s}(x)\right|^{2}\right) d s\right] \\
& \leq m+\bar{C}+\mathrm{E}_{Q}\left[\int_{0}^{T \wedge \sigma_{m}} \int_{\mathbb{R} \backslash\{0\}} \exp \left(4|| J \|_{S^{\infty}}^{2}\right)\left|\tilde{Z}_{s}(x)\right|^{4} d s\right] \\
& \leq m+\bar{C}+4\|J\|_{S^{\infty}}^{2} \exp \left(4|| J \|_{S^{\infty}}^{2}\right) \mathrm{E}_{Q}\left[\int_{0}^{T \wedge \sigma_{m}}\left|\tilde{Z}_{s}(x)\right|^{2} d s\right] \\
& <m+\bar{C}+4\|J\|_{S^{\infty}}^{2} \exp \left(4|| J \|_{S^{\infty}}^{2}\right) m,
\end{aligned}
$$

with $\Phi(x)=e^{x}-x-1$. In the first inequality, we used the definition of $\sigma_{m}$. In the second inequality, we applied (A.1). In the third inequality, we used (A.10). In the fourth inequality, we used (A.3) and that $|\tilde{Z}|^{2}$ is bounded by $4\|J\|_{S^{\infty}}^{2}$. In the fifth inequality, we applied again that $|\tilde{Z}|^{2}$ is bounded by $4\|J\|_{S^{\infty}}^{2}$. In the last inequality, we used the definition of $\sigma_{m}$. It follows that indeed $\left(\tilde{Z} \cdot \tilde{N}^{Q}\right)_{t \wedge \sigma_{m}}$ is a square-integrable $Q$-martingale. Therefore,

$$
M_{t}^{Q}:=M_{t}-\int_{0}^{t} Z_{s} q_{s} d s-\int_{0}^{t} \int_{\mathbb{R} \backslash\{0\}} \tilde{Z}_{s}(x) \psi_{s}(x) n_{p}(s, d x) d s=\left(Z \cdot W^{Q}\right)_{t}+\left(\tilde{Z} \cdot \tilde{N}^{Q}\right)_{t}
$$

is a locally square-integrable martingale with local stopping times $\sigma_{m}$. Next, choose $C$ as in Lemma A.16 below (with $\bar{A}=2\|J\|_{S^{\infty}}$ ). Note that, by (A.1),

$$
-\psi(x) \tilde{z}(x)=-C\left(\psi(x) \frac{\tilde{z}(x)}{C}\right) \geq-C \Psi(\psi(x))-C \Phi\left(\frac{\tilde{z}(x)}{C}\right) .
$$


Now, by Itô's generalized formula, for any stopping times $\sigma$ and $\sigma_{m}$ we have that $Q$-a.s.

$$
\begin{aligned}
\exp \left(J_{T \wedge \sigma_{m}}\right)= & \exp \left(J_{\sigma \wedge \sigma_{m}}\right)-\int_{\sigma \wedge \sigma_{m}}^{T \wedge \sigma_{m}} \exp \left(J_{s-}\right) d M_{s}+\int_{\sigma \wedge \sigma_{m}}^{T \wedge \sigma_{m}} \exp \left(J_{s-}\right) d A_{s} \\
& +\int_{\sigma \wedge \sigma_{m}}^{T \wedge \sigma_{m}} \exp \left(J_{s-}\right)\left(\frac{1}{2}\left|Z_{s}\right|^{2}+\int_{\mathbb{R} \backslash\{0\}}\left(\exp \left\{-\tilde{Z}_{s}(x)\right\}-1+\tilde{Z}_{s}(x)\right) n_{p}(s, d x)\right) d s \\
= & \exp \left(J_{\sigma \wedge \sigma_{m}}\right)-\int_{\sigma \wedge \sigma_{m}}^{T \wedge \sigma_{m}} \exp \left(J_{s-}\right) d M_{s}^{Q}+\int_{\sigma \wedge \sigma_{m}}^{T \wedge \sigma_{m}} \exp \left(J_{s-}\right) d A_{s} \\
& +\int_{\sigma \wedge \sigma_{m}}^{T \wedge \sigma_{m}} \exp \left(J_{s-}\right)\left(\frac{1}{2}\left|Z_{s}\right|^{2}-q_{s} Z_{s}-\int_{\mathbb{R} \backslash\{0\}} \tilde{Z}_{s}(x) \psi_{s}(x) n_{p}(s, d x)\right. \\
& \left.+\int_{\mathbb{R} \backslash\{0\}}\left(\exp \left\{-\tilde{Z}_{s}(x)\right\}-1+\tilde{Z}_{s}(x)\right) n_{p}(s, d x)\right) d s .
\end{aligned}
$$

Taking conditional expectations on both sides in (A.13) yields

$$
\begin{aligned}
& \mathrm{E}_{Q}\left[\exp \left(J_{T \wedge \sigma_{m}}\right) \mid \mathcal{F}_{\sigma \wedge \sigma_{m}}\right] \\
& =\exp \left(J_{\sigma \wedge \sigma_{m}}\right)+\mathrm{E}_{Q}\left[\int_{\sigma \wedge \sigma_{m}}^{T \wedge \sigma_{m}} \exp \left(J_{s-}\right) d A_{s}+\int_{\sigma \wedge \sigma_{m}}^{T \wedge \sigma_{m}} \exp \left(J_{s-}\right)\left(\frac{1}{2}\left|Z_{s}\right|^{2}-q_{s} Z_{s}\right.\right. \\
& \left.\left.\quad+\int_{\mathbb{R} \backslash\{0\}}\left[-\tilde{Z}_{s}(x) \psi_{s}(x)+\Phi\left(-\tilde{Z}_{s}(x)\right)\right] n_{p}(s, d x)\right) d s \mid \mathcal{F}_{\sigma \wedge \sigma_{m}}\right] .
\end{aligned}
$$

As $J$ is bounded by $\tilde{C},($ A.14) entails that

$$
\begin{aligned}
& \mathrm{E}_{Q}\left[\exp \left(J_{T \wedge \sigma_{m}}\right) \mid \mathcal{F}_{\sigma \wedge \sigma_{m}}\right] \\
& \geq \exp (-\tilde{C})+\mathrm{E}_{Q}\left[e^{-\tilde{C}}\left(A_{T \wedge \sigma_{m}}-A_{\sigma \wedge \sigma_{m}}\right)\right. \\
& \left.+\int_{\sigma \wedge \sigma_{m}}^{T \wedge \sigma_{m}} \exp \left(J_{s-}\right)\left(\frac{1}{4}\left|Z_{s}\right|^{2}-4\left|q_{s}\right|^{2}+\int_{\mathbb{R} \backslash\{0\}}\left[-\tilde{Z}_{s}(x) \psi_{s}(x)+\Phi\left(-\tilde{Z}_{s}(x)\right)\right] n_{p}(s, d x)\right) d s \mid \mathcal{F}_{\sigma \wedge \sigma_{m}}\right] \\
& \geq \exp (-\tilde{C})+\mathrm{E}_{Q}\left[e^{-\tilde{C}}\left(A_{T \wedge \sigma_{m}}-A_{\sigma \wedge \sigma_{m}}\right)+\int_{\sigma \wedge \sigma_{m}}^{T \wedge \sigma_{m}}\left(\frac{e^{-\tilde{C}}}{4}\left|Z_{s}\right|^{2}-4 e^{\tilde{C}}\left|q_{s}\right|^{2}\right.\right. \\
& \left.\left.+e^{J_{s-}} \int_{\mathbb{R} \backslash\{0\}}\left\{-C \Psi\left(\psi_{s}(x)\right)-C \Phi\left(\frac{\tilde{Z}_{s}(x)}{C}\right)+\Phi\left(-\tilde{Z}_{s}(x)\right)\right\} n_{p}(s, d x)\right) d s \mid \mathcal{F}_{\sigma \wedge \sigma_{m}}\right] \\
& \geq \exp (-\tilde{C})+\mathrm{E}_{Q}\left[e^{-\tilde{C}}\left(A_{T \wedge \sigma_{m}}-A_{\sigma \wedge \sigma_{m}}\right)+\int_{\sigma \wedge \sigma_{m}}^{T \wedge \sigma_{m}}\left(\frac{e^{-\tilde{C}}}{4}\left|Z_{s}\right|^{2}-4 e^{\tilde{C}}\left|q_{s}\right|^{2}\right.\right. \\
& \left.\left.+\int_{\mathbb{R} \backslash\{0\}}\left[-C e^{\tilde{C}} \Psi\left(\psi_{s}(x)\right)+B e^{-\tilde{C}}\left|\tilde{Z}_{s}(x)\right|^{2}\right] n_{p}(s, d x)\right) d s \mid \mathcal{F}_{\sigma \wedge \sigma_{m}}\right],
\end{aligned}
$$

where $B>0$ in the last inequality stems from Lemma A.16. In the first inequality, we used $|a b| \leq 4 a^{2}+\frac{b^{2}}{4}$ for the term $q_{s} Z_{s}$. The second inequality holds by (A.12). In the last inequality, we applied Lemma A.16 and the fact that $\tilde{Z}$ is bounded by $2\|J\|_{\infty}$. Now to see that $Z$ is a $\operatorname{BMO}(P)$ process, note that for $Q=P$, we have that $q=\psi=0$. (A.15) implies then that there exist a constant $C^{\prime}>0$ only depending on $\tilde{C}$ and $B$, such that for every stopping time $\sigma$ and 
$\sigma_{m}$,

$$
\mathrm{E}\left[A_{T \wedge \sigma_{m}}-A_{\sigma \wedge \sigma_{m}}+\int_{\sigma \wedge \sigma_{m}}^{T \wedge \sigma_{m}}\left(\left|Z_{s}\right|^{2}+\int_{\mathbb{R} \backslash\{0\}}\left|\tilde{Z}_{s}(x)\right|^{2} n_{p}(s, d x)\right) d s \mid \mathcal{F}_{\sigma \wedge \sigma_{m}}\right] \leq C^{\prime} .
$$

Choosing $\sigma=0$ and letting $m$ converge to infinity yields

$$
\mathrm{E}\left[A_{T}+\int_{0}^{T}\left(\left|Z_{s}\right|^{2}+\int_{\mathbb{R} \backslash\{0\}}\left|\tilde{Z}_{s}(x)\right|^{2} n_{p}(s, d x)\right) d s\right] \leq C^{\prime},
$$

where we used the monotone convergence theorem. (Recall that $A_{0}=0$.) Now (A.17) implies that $Z \in L^{2}(d P \times d s)$ and $\tilde{Z} \in L^{2}\left(d P \times n_{p}(s, d x) \times d s\right)$. Therefore, $M$ is a true martingale and we may choose $\sigma_{m}=T$. But then (A.16) yields that

$$
\mathrm{E}\left[A_{T}-A_{\sigma}+\int_{\sigma}^{T}\left(\left|Z_{s}\right|^{2}+\int_{\mathbb{R} \backslash\{0\}}\left|\tilde{Z}_{s}(x)\right|^{2} n_{p}(s, d x)\right) d s \mid \mathcal{F}_{\sigma}\right] \leq C^{\prime} .
$$

As $A_{T}-A_{\sigma} \geq 0$ (since $A$ is increasing), it follows that $Z$ is a $\operatorname{BMO}(P)$ process and $\tilde{Z}$ is a $\operatorname{BMO}(P)$ function.

For the second part of the lemma, let $Q \in C_{k}$. It follows from (A.10) and (A.15) with $\sigma=0$ that there exists a constant $\hat{C}$ such that for every $\sigma_{m}$,

$$
\mathrm{E}_{Q}\left[A_{T \wedge \sigma_{m}}+\int_{0}^{T \wedge \sigma_{m}}\left(\left|Z_{s}\right|^{2}+\int_{\mathbb{R} \backslash\{0\}}\left|\tilde{Z}_{s}(x)\right|^{2} n_{p}(s, d x)\right) d s\right] \leq \hat{C} .
$$

Letting $m$ converge to infinity and using the monotone convergence theorem (A.18) yields

$$
\mathrm{E}_{Q}\left[A_{T}+\int_{0}^{T}\left(\left|Z_{s}\right|^{2}+\int_{\mathbb{R} \backslash\{0\}}\left|\tilde{Z}_{s}(x)\right|^{2} n_{p}(s, d x)\right) d s\right] \leq \hat{C} .
$$

This shows that $A_{T} \in L^{1}(Q), Z \in L^{2}(d Q \times d s)$ and $\tilde{Z} \in L^{2}\left(d Q \times n_{p}(s, d x) \times d s\right)$. What is left to show is that $\tilde{Z} \in L^{2}\left(d Q \times\left(1+\psi_{s}(x)\right) n_{p}(s, d x) \times d s\right)$. First of all note that clearly $x^{4} \leq 4\|J\|_{S^{\infty}}^{2} x^{2}$ for all $x \in \mathbb{R}$ with $|x| \leq 2\|J\|_{S^{\infty}}$. Hence, by (A.1) and (A.2),

$$
\begin{aligned}
\left|\tilde{Z}_{s}(x)\right|^{2} \psi_{s}(x) & \leq \Phi\left(\left|\tilde{Z}_{s}(x)\right|^{2}\right)+\Psi\left(\psi_{s}(x)\right) \\
& \leq \exp \left\{4\|J\|_{S^{\infty}}^{2}\right\}\left|\tilde{Z}_{s}(x)\right|^{4}+\Psi\left(\psi_{s}(x)\right) \\
& =4|| J \|_{S^{\infty}}^{2} \exp \left\{4|| J \|_{S^{\infty}}^{2}\right\}\left|\tilde{Z}_{s}(x)\right|^{2}+\Psi\left(\psi_{s}(x)\right) .
\end{aligned}
$$

Now we have already shown that $\left|\tilde{Z}_{s}(x)\right|^{2} \in L^{1}\left(d Q \times n_{p}(s, d x) \times d s\right)$. On the other hand, $\Psi\left(\psi_{s}(x)\right) \in L^{1}\left(d Q \times n_{p}(s, d x) \times d s\right)$ because of (A.10). Therefore, it follows from (A.19) that $\left|\tilde{Z}_{s}(x)\right|^{2}\left(1+\psi_{s}(x)\right) \in L^{1}\left(d Q \times n_{p}(s, d x) \times d s\right)$, so that indeed $\tilde{Z} \in L^{2}\left(d Q \times n_{p}^{Q}(s, d x) \times d s\right)$.

Remark A.15 Suppose that $J=J_{0}+A-M$, where, rather than assuming that $A$ is increasing, we assume that there exists a constant $b$ such that $A_{t}+b t$ is increasing. This would be the case if $A$ is given as the integral of a driver function bounded from below. In this case the conclusions of Lemma A.14 still hold. This can be seen by defining, $\bar{J}_{t}:=J_{0}+A_{t}+b t-M_{t}$. Then $A_{t}+b t$, the predictable part of $\bar{J}$, is increasing. As $\bar{J}$ is bounded, we can apply Lemma A.14 to $\bar{J}$ in order to obtain the integrability results on $Z$ and $\tilde{Z}$. 
Lemma A.16 Let $\bar{A}>0$. There exist $C, B>0$ such that for all $x \in[-\bar{A}, \bar{A}]$,

$$
\Phi(-x)-C \Phi\left(\frac{x}{C}\right) \geq B|x|^{2} .
$$

Proof. By (A.2)-(A.3),

$$
\Phi(-x)-C \Phi\left(\frac{x}{C}\right) \geq\left(\frac{1}{2 e^{\bar{A}}}-\frac{1}{C} \exp \left\{\frac{\bar{A}}{C}\right\}\right) x^{2} .
$$

If we choose $C$ large enough, then $B:=\frac{1}{2 e^{A}}-\frac{1}{C} \exp \left\{\frac{\bar{A}}{C}\right\}>0$.

Proposition A.17 For every $Q \ll P$ we have that

$$
H(Q \mid P)=\mathrm{E}_{Q}\left[\int_{0}^{T} \frac{1}{2}\left|q_{s}\right|^{2}+\int_{\mathbb{R} \backslash\{0\}}\left[\left(1+\psi_{s}(x)\right) \log \left(1+\psi_{s}(x)\right)-\psi_{s}(x)\right] n_{p}(s, d x) d s\right] .
$$

Proof. As both sides are non-negative, it is sufficient to prove (A.20) if either the left- or the right-hand side is finite. First of all assume that we have $Q$ such that $H(Q \mid P)<\infty$. If $Q$ is equivalent to $P$, (A.20) corresponds to Proposition 9.10 in Cont and Tankov [15]. If $Q$ is not equivalent to $P$, let $0 \leq \lambda \leq 1$ and define $Q^{\lambda}=\lambda Q+(1-\lambda) P$. It is not hard to see using the dominated convergence theorem that $H\left(Q^{\lambda} \mid P\right) \stackrel{\lambda \rightarrow 1}{\rightarrow} H(Q \mid P)$. On the other hand, a similar argument as in (3.2) yields that the density process of $Q^{\lambda}$ is equal to $\mathcal{E}\left(\left(q^{\lambda} \cdot W\right)_{t}+\left(\psi^{\lambda} \cdot \tilde{N}_{p}\right)_{t}\right)$ with

$$
q_{t}^{\lambda}=\frac{\lambda D_{t} q_{t}}{\lambda D_{t}+(1-\lambda)} I_{\{t \leq \tau\}} \quad \text { and } \quad \psi_{t}^{\lambda}=\frac{\lambda D_{t} \psi_{t}}{\lambda D_{t}+(1-\lambda)} I_{\{t \leq \tau\}}
$$

Clearly, for every $\omega$ and $s,\left(q_{s}^{\lambda}(\omega)\right)_{\lambda}$ and $\left(\psi_{s}^{\lambda}(\omega)\right)_{\lambda}$ are increasing (decreasing) in $\lambda \in[0,1]$ on their respective positive (negative) parts. Furthermore, they converge to $q_{s}$ and $\psi_{s}$, respectively, as $\lambda$ tends to one. Therefore, indeed

$$
\begin{aligned}
H(Q \mid P) & =\lim _{\lambda \rightarrow 1} H\left(Q^{\lambda} \mid P\right) \\
& =\lim _{\lambda \rightarrow 1} \mathrm{E}_{Q}\left[\int_{0}^{T}\left(\frac{1}{2}\left|q_{s}^{\lambda}\right|^{2}+\int_{\mathbb{R} \backslash\{0\}} \Psi\left(\psi_{s}^{\lambda}(x)\right) n_{p}(s, d x)\right) d s\right] \\
& =\mathrm{E}_{Q}\left[\int_{0}^{T}\left(\frac{1}{2}\left|q_{s}\right|^{2}+\int_{\mathbb{R} \backslash\{0\}} \Psi\left(\psi_{s}(x)\right) n_{p}(s, d x)\right) d s\right],
\end{aligned}
$$

where we applied the monotone convergence theorem in the last equality.

Next, suppose that we have a $Q$ with corresponding $(q, \psi)$ such that the RHS in (A.20) is finite. Now clearly $\psi_{m, s}(x)=\psi_{s}(x) I_{\left\{\psi_{s}(x) \leq m\right\}} I_{\{|x| \geq 1 / m\}}(x)$ is in $L^{2}\left(d Q \times n^{Q}(s, d x) \times d s\right)$. 
Taking the logarithm of the Radon-Nikodym derivative yields that $Q$-a.s.

$$
\begin{aligned}
\log \left(\frac{d Q}{d P}\right)= & \lim _{m} \log \left\{\mathcal{E}\left(\int_{0}^{T} q_{s} d W_{s}+\int_{0}^{T} \int_{\mathbb{R} \backslash\{0\}} \psi_{m, s}(x) \tilde{N}_{p}(d s, d x)\right)\right\} \\
= & \lim _{m}\left\{\int_{0}^{T} q_{s} d W_{s}-\int_{0}^{T} \frac{1}{2}\left|q_{s}\right|^{2} d s+\int_{0}^{T} \int_{\mathbb{R} \backslash\{0\}} \psi_{m, s}(x) \tilde{N}_{p}(d s, d x)\right. \\
& \left.+\int_{0}^{T} \int_{\mathbb{R} \backslash\{0\}}\left(\log \left(1+\psi_{m, s}(x)\right)-\psi_{m, s}(x)\right) N_{p}(d s, d x)\right\} \\
= & \lim _{m}\left\{\int_{0}^{T} q_{s} d W_{s}^{Q}+\int_{0}^{T} \frac{1}{2}\left|q_{s}\right|^{2} d s+\int_{0}^{T} \int_{\mathbb{R} \backslash\{0\}} \psi_{m, s}(x) \tilde{N}_{p}^{Q}(d s, d x)\right. \\
& +\int_{0}^{T} \int_{\mathbb{R} \backslash\{0\}}\left(\log \left(1+\psi_{m, s}(x)\right)-\psi_{m, s}(x)\right) \tilde{N}_{p}^{Q}(d s, d x) \\
& \left.+\int_{0}^{T} \int_{\mathbb{R} \backslash\{0\}}\left[\psi_{m, s}(x) \psi_{s}(x)+\left(1+\psi_{s}(x)\right)\left\{\log \left(1+\psi_{m, s}(x)\right)-\psi_{m, s}(x)\right\}\right] n_{p}(s, d x) d s\right\} \\
= & \lim _{m}\left\{\int_{0}^{T} q_{s} d W_{s}^{Q}+\int_{0}^{T} \frac{1}{2}\left|q_{s}\right|^{2} d s+\int_{0}^{T} \int_{\mathbb{R} \backslash\{0\}} \log \left(1+\psi_{m, s}(x)\right) \tilde{N}_{p}^{Q}(d s, d x)\right. \\
& \left.+\int_{0}^{T} \int_{\mathbb{R} \backslash\{0\}}\left[\left(1+\psi_{s}(x)\right) \log \left(1+\psi_{m, s}(x)\right)-\psi_{m, s}(x)\right] n_{p}(s, d x) d s\right\},
\end{aligned}
$$

where we used in the second equality that, for fixed $\omega$, by the definition of $\psi_{m}$, we have that $\left(1+\psi_{s}\right)\left\{\log \left(1+\psi_{m, s}\right)-\psi_{m, s}\right\} \in L^{1}\left(n_{p}(s, d x) \times d s\right)$. Thus, $\log \left(1+\psi_{m, s}\right)-\psi_{m, s} \in L^{1}\left(n_{p}^{Q}(s, d x) \times\right.$ $d s)$. In particular, $\int_{0}^{T} \int_{\mathbb{R} \backslash\{0\}}\left(\log \left(1+\psi_{m, s}(x)\right)-\psi_{m, s}(x)\right) \tilde{N}_{p}^{Q}(d s, d x)$ is well-defined.

Now Lemma A.18 below yields that each of the processes

$$
\begin{aligned}
M_{t}^{\prime}: & =\int_{0}^{t} q_{s} d W_{s}^{Q}, \quad M_{m, t}^{\prime \prime}:=\int_{0}^{t} \int_{\mathbb{R} \backslash\{0\}} \log \left(1+\psi_{m, s}(x)\right) \tilde{N}_{p}^{Q}(d s, d x), \\
M_{t}^{\prime \prime}: & =\int_{0}^{t} \int_{\mathbb{R} \backslash\{0\}} \log \left(1+\psi_{s}(x)\right) \tilde{N}_{p}^{Q}(d s, d x),
\end{aligned}
$$

is a martingale, and $M_{m}^{\prime \prime}$ converges in $L^{1}(Q)$ to $M^{\prime \prime}$. By switching to a subsequence, we may assume that the convergence holds a.s. Finally, by the monotone convergence theorem, the last term in (A.21) converges to $\int_{0}^{T} \int_{\mathbb{R} \backslash\{0\}} \Psi\left(\psi_{s}(x)\right) n_{p}(s, d x) d s Q$ a.s. Thus, (A.21) yields that

$$
\begin{aligned}
\log \left(\frac{d Q}{d P}\right)=\int_{0}^{T} & q_{s} d W_{s}^{Q}+\int_{0}^{T} \frac{1}{2}\left|q_{s}\right|^{2} d s+\int_{0}^{T} \int_{\mathbb{R} \backslash\{0\}} \log \left(1+\psi_{s}(x)\right) \tilde{N}_{p}^{Q}(d s, d x) \\
& +\int_{0}^{T} \int_{\mathbb{R} \backslash\{0\}}\left[\left(1+\psi_{s}(x)\right) \log \left(1+\psi_{s}(x)\right)-\psi_{s}(x)\right] n_{p}(s, d x) d s .
\end{aligned}
$$

Taking the expectation in (A.23) with respect to $Q$, and using that by Lemma A.18 $M^{\prime}$ and $M^{\prime \prime}$ are martingales, (A.20) follows.

Lemma A.18 Let $Q \ll P$ be such that the RHS in (A.20) is finite. Then the stochastic processes $M^{\prime}$ and $M^{\prime \prime}$ defined in (A.22) are martingales. Furthermore, $M_{m, T}^{\prime \prime}$ converges in $L^{1}(Q)$ to $M_{T}^{\prime \prime}$. 
Proof. First of all note that, as the RHS in (A.20) is finite, we have $\mathrm{E}_{Q}\left[\int_{0}^{T}\left|q_{s}\right|^{2} d s\right]<\infty$. Therefore, $M^{\prime}$ is a martingale. Let us prove that $M^{\prime \prime}$ is also a martingale. We write

$$
\Psi(x)=(1+x) \log (1+x)-x \geq \frac{1}{6}(1+x) \log ^{2}(1+x) \geq 0 \quad \text { for }-1 \leq x \leq e^{2}-1 .
$$

(This may be seen by noticing that at zero both sides are equal to zero and their derivatives are equal to zero, too. Furthermore, the second derivative of the LHS is larger than the second derivative of the RHS for $-1 \leq x \leq e^{2}-1$.) As the RHS in (A.20) is finite so that $\Psi\left(\psi_{s}(x)\right) \in L^{1}\left(d Q \times n_{p}(s, d x) \times d x\right)$, we obtain

$$
\left(1+\psi_{s}(x)\right) \log ^{2}\left(1+\psi_{s}(x)\right) I_{\left\{\psi_{s}(x) \leq e^{2}-1\right\}}(x) \in L^{1}\left(d Q \times n_{p}(s, d x) \times d s\right) .
$$

This implies that

$$
\log \left(1+\psi_{s}(x)\right) I_{\left\{\psi_{s}(x) \leq e^{2}-1\right\}}(x) \in L^{2}\left(d Q \times n_{p}^{Q}(s, d x) \times d s\right) .
$$

Furthermore, the positive (negative) parts of $\log \left(1+\psi_{m, s}(x)\right) I_{\left\{\psi_{m, s}(x) \leq e^{2}-1\right\}}(x)$ increase (decrease) to those of $\log \left(1+\psi_{s}(x)\right) I_{\left\{\psi_{s}(x) \leq e^{2}-1\right\}}(x)$ as $m$ tends to infinity. By the monotone convergence theorem, this yields that

$\log \left(1+\psi_{m, s}(x)\right) I_{\left\{\psi_{m, s}(x) \leq e^{2}-1\right\}}(x) \stackrel{m \rightarrow \infty}{\rightarrow} \log \left(1+\psi_{s}(x)\right) I_{\left\{\psi_{s}(x) \leq e^{2}-1\right\}}(x)$ in $L^{2}\left(d Q \times n_{p}^{Q}(s, d x) \times d s\right)$.

Therefore,

$$
\begin{aligned}
\bar{M}_{m, t}: & =\int_{0}^{t} \int_{\mathbb{R} \backslash\{0\}} \log \left(1+\psi_{m, s}(x)\right) I_{\left\{\psi_{m, s}(x) \leq e^{2}-1\right\}}(x) \tilde{N}^{Q}(d s, d x) \\
\bar{M}_{t}: & =\int_{0}^{t} \int_{\mathbb{R} \backslash\{0\}} \log \left(1+\psi_{s}(x)\right) I_{\left\{\psi_{s}(x) \leq e^{2}-1\right\}}(x) \tilde{N}^{Q}(d s, d x)
\end{aligned}
$$

are martingales in $L^{2}(Q)$, and $\bar{M}_{m, T}$ converges to $\bar{M}_{T}$ in $L^{2}(Q)$. Next, note that

$$
(1+x) \log (1+x) \leq 2((1+x) \log (1+x)-x) \quad \text { for } x>e^{2}-1 .
$$

(This may be seen by noticing that the inequality holds for $x=e^{2}-1$, and that the derivative of the RHS is larger than the derivative of the LHS for $x>e^{2}-1$.) As the RHS in (A.20) is finite, it follows that $\left(1+\psi_{s}(x)\right) \log \left(1+\psi_{s}(x)\right) I_{\left\{\psi_{s}(x)>e^{2}-1\right\}}(x) \in L^{1}\left(d Q \times n_{p}(s, d x) \times d s\right)$, so that

$$
\log \left(1+\psi_{s}(x)\right) I_{\left\{\psi_{s}(x)>e^{2}-1\right\}}(x) \in L^{1}\left(d Q \times n_{p}^{Q}(s, d x) \times d s\right) .
$$

Moreover, $\log \left(1+\psi_{m, s}(x)\right) I_{\left\{\psi_{m, s}(x)>e^{2}-1\right\}}(x)$ increases to $\log \left(1+\psi_{s}(x)\right) I_{\left\{\psi_{s}(x)>e^{2}-1\right\}}(x)$ as $m$ tends to infinity. By the monotone convergence theorem, it follows that

$\log \left(1+\psi_{m, s}(x)\right) I_{\left\{\psi_{m, s}(x)>e^{2}-1\right\}}(x) \stackrel{m \rightarrow \infty}{\rightarrow} \log \left(1+\psi_{s}(x)\right) I_{\left\{\psi_{s}(x)>e^{2}-1\right\}}(x)$ in $L^{1}\left(d Q \times n_{p}^{Q}(s, d x) \times d s\right)$.

Consequently, by the definition of a compensator, the processes

$$
\begin{aligned}
\hat{M}_{m, t} & :=\int_{0}^{t} \int_{\mathbb{R} \backslash\{0\}} \log \left(1+\psi_{m, s}(x)\right) I_{\left\{\psi_{m, s}(x)>e^{2}-1\right\}}(x) \tilde{N}^{Q}(d s, d x) \\
\hat{M}_{t} & :=\int_{0}^{t} \int_{\mathbb{R} \backslash\{0\}} \log \left(1+\psi_{s}(x)\right) I_{\left\{\psi_{s}(x)>e^{2}-1\right\}}(x) \tilde{N}^{Q}(d s, d x)
\end{aligned}
$$

are both martingales, see, for instance, Jacod and Shiryaev [40], Ch. II, Th. 1.8(i). Furthermore, $\hat{M}_{m, T}$ converges in $L^{1}(Q)$ to $\hat{M}_{T}$. As $M^{\prime \prime}=\bar{M}+\hat{M}$ and $M_{m}^{\prime \prime}=\bar{M}_{m}^{1}+\hat{M}_{m}$ the proposition now follows. 
Lemma A.19 Let $Z$ be a $B M O(P)$ process and let $\tilde{Z}$ be a $B M O(P)$ function. Suppose that $g_{1}$ and $g_{2}$ satisfy (c)-(e) and that we have a measure $Q \ll P$ with corresponding $q$ and $\psi$ such that $q_{t} \in \partial g_{1}\left(t, Z_{t}\right), d Q \times d t$ a.s, and $\psi_{t}(x) \in \partial g_{2}\left(t, x, \tilde{Z}_{t}(x)\right), d Q \times n_{p}(t, d x) \times d t$ a.s. Then $Q \sim P$.

Proof. Let $D_{t}=\mathrm{E}\left[\frac{d Q}{d P} \mid \mathcal{F}_{t}\right]$ and $\tau=\inf \left\{t \in[0, T] \mid D_{t}=0\right\} \wedge T$. As $q_{t} \in \partial g_{1}\left(t, Z_{t}\right) d Q \times d t$ a.s, $\psi_{t}(x) \in \partial g_{2}\left(t, x, \tilde{Z}_{t}(x)\right) d Q \times n_{p}(t, d x) \times d t$ a.s. and $\tilde{Z}$ is bounded, by properties (d)-(e), there exist constants $\bar{K}_{1}, \bar{K}_{2}>0$ and a $\operatorname{BMO}(P)$ function $\tilde{H}$, such that for Lebesgue a.s. all $t \leq \tau$,

$$
\left|q_{t}\right| \leq \bar{K}_{1}\left(1+\left|Z_{t}\right|\right) \quad \text { and } \quad\left|\psi_{t}(x)\right| \leq \tilde{H}_{t}(x)+\bar{K}_{2}\left|\tilde{Z}_{t}(x)\right| .
$$

Since, by assumption, $Z$ is a $\operatorname{BMO}(P)$ process, and $\tilde{Z}$ and $\tilde{H}$ are $\operatorname{BMO}(P)$ functions, (A.24) entails that $\left(q_{t \wedge \tau}\right)$ is a $\mathrm{BMO}(P)$ process and $\left(\psi_{t \wedge \tau}\right)$ is a $\mathrm{BMO}(P)$ function. Furthermore, property (e) implies that $\psi_{t \wedge \tau} \geq-1+\epsilon$ for an $\epsilon>0$. But then $M_{t}:=(q \cdot W)_{t \wedge \tau}+\left(\psi \cdot \tilde{N}_{p}\right)_{t \wedge \tau}$ is a $\operatorname{BMO}(P)$ martingale with $\Delta M_{t} \geq-1+\epsilon$. Since $\frac{d Q}{d P}=\mathcal{E}\left(M_{T}\right)$, Theorem A.4 implies that $\frac{d Q}{d P}>0$ and $\tau=T, P$-a.s.

The next lemma can be proved in the same way using (A.24) and Theorem A.4 with $\tau$ replaced by $T$.

Lemma A.20 Let $Z$ and $\tilde{Z}$ be a $B M O(P)$ process and a $B M O(P)$ function, respectively. Suppose that $g$ satisfies (c)-(e) and we have predictable $q$ and $\psi$ satisfying $q_{t} \in \partial g_{1}\left(t, Z_{t}\right) d P \times d t$ a.s. and $\psi_{t}(x) \in \partial g_{2}\left(t, x, \tilde{Z}_{t}(x)\right), d P \times n_{p}(t, d x) \times d t$ a.s. Then we have that the measure $Q$ induced by $\frac{d Q}{d P}:=\mathcal{E}\left((q \cdot W)_{T}+\left(\psi \cdot \tilde{N}_{p}\right)_{T}\right)$ is well-defined and equivalent to $P$.

The following proposition is a key step in the proof of Theorem 4.1.

Theorem A.21 Suppose that $g_{1}$ and $g_{2}$ satisfy properties (a)-(e) above with certain constants $\bar{K}, K_{2}, \bar{K}_{1}, \bar{K}_{2}>0$ and a non-negative $B M O(P)$ function $\tilde{H}_{s}(x)$. Let $r_{1}$ and $r_{2}$ be the dual conjugates of $g_{1}$ and $g_{2}$, respectively. Define $U_{t}(F)$ by (A.8). Then $U_{t}(F)$ is the unique solution to the BSDE (4.4).

Proof. Denote $r(t, q, \psi):=r_{1}(t, q)+\int_{\mathbb{R} \backslash\{0\}} r_{2}(t, x, \psi(x)) n_{p}(t, d x)$. From the decomposition (A.9) and Lemma A.14 it follows that, for every $Q \ll P$, there exist a $\operatorname{BMO}(P)$ process $Z$ and a $\operatorname{BMO}(P)$ function $\tilde{Z}$ such that

$$
\begin{aligned}
d U_{t}(F)+r\left(t, q_{t}, \psi_{t}\right) d t & =d A_{t}-Z_{t} d W_{t}-\int_{\mathbb{R} \backslash\{0\}} \tilde{Z}_{t}(x) \tilde{N}_{p}(d t, d x)+r\left(t, q_{t}, \psi_{t}\right) d t \\
=d A_{t}+ & {\left[-q_{t} Z_{t}-\int_{\mathbb{R} \backslash\{0\}} \tilde{Z}_{t}(x) \psi_{t}(x) n_{p}(t, d x)\right.} \\
& \left.+r\left(t, q_{t}, \psi_{t}\right)\right] d t-Z_{t} d W_{t}^{Q}-\int_{\mathbb{R} \backslash\{0\}} \tilde{Z}_{t}(x) \tilde{N}_{p}^{Q}(d t, d x),
\end{aligned}
$$

for Lebesgue a.s. all $t \in[0, \tau]$. By Lemma A.13(1), $U_{t}(F)+\int_{0}^{t} r\left(s, q_{s}, \psi_{s}\right) d s$ is a $Q$-submartingale on $[0, T]$. (Recall that $\tau=T Q$ a.s.) Thus, for every $Q$,

$$
d A_{t} \geq\left[q_{t} Z_{t}+\int_{\mathbb{R} \backslash\{0\}} \tilde{Z}_{t}(x) \psi_{t}(x) n_{p}(t, d x)-r\left(t, q_{t}, \psi_{t}\right)\right] d t, \quad Q \text {-a.s. }
$$


By Lemma A.31 below, we may choose a predictable $q_{t} \in \partial g_{1}\left(t, Z_{t}\right)$. Set $\psi_{t}(x):=g_{2,+}^{\prime}\left(t, x, \tilde{Z}_{t}(x)\right)$, where $g_{2,+}^{\prime}$ denotes the right-hand side derivative of $g_{2}$ with respect to its last component. By Lemma A.20, the corresponding measure $Q$ is well-defined and $Q \sim P$. Plugging $q$ and $\psi$ into (A.26), we get

$$
d A_{t} \geq\left[g_{1}\left(t, Z_{t}\right)+\int_{\mathbb{R} \backslash\{0\}} g_{2}\left(t, x, \tilde{Z}_{t}(x)\right) n_{p}(t, d x)\right] d t, \quad Q \text {-a.s. }
$$

As $Q \sim P$ this implies that

$$
d A_{t} \geq\left[g_{1}\left(t, Z_{t}\right)+\int_{\mathbb{R} \backslash\{0\}} g_{2}\left(t, x, \tilde{Z}_{t}(x)\right) n_{p}(t, d x)\right] d t, \quad P \text {-a.s. }
$$

Next, note that since $g$ satisfies property (b), Lemma A.10 and Proposition A.17 yield that, for any $k>0$, there exists a $k^{\prime}>0$ such that

$$
\begin{aligned}
C_{k} & =\left\{Q \ll P \mid \mathrm{E}_{Q}\left[\int_{0}^{T}\left[r_{1}\left(s, q_{s}\right)+\int_{\mathbb{R} \backslash\{0\}} r_{2}\left(s, x, \psi_{s}(x)\right) n_{p}(s, d x)\right] d s\right] \leq k\right\} \\
& \subset\left\{Q \ll P \mid \mathrm{E}_{Q}\left[\log \left(\frac{d Q}{d P}\right)\right] \leq k^{\prime}\right\} .
\end{aligned}
$$

By the Dunford-Pettis theorem, this implies that $C_{k}$ is weakly compact. Thus, for $k$ large enough, the infimum in (A.8) is attained in a $Q \in C_{k}$ for $t=0$. Again, let $D_{t}=\mathrm{E}\left[\frac{d Q}{d P} \mid \mathcal{F}_{t}\right]$ and $\tau=\inf \left\{t \geq 0 \mid D_{t}=0\right\} \wedge T$. From (A.25) and the fact that, by Lemma A.13(2), $U_{t}(F)+$ $\int_{0}^{t} r\left(s, q_{s}, \psi_{s}\right) d s$ is a $Q$-martingale on $[0, T]$ (as $\tau=T Q$-a.s.), it follows that

$$
d A_{t}=\left[q_{t} Z_{t}+\int_{\mathbb{R} \backslash\{0\}} \tilde{Z}_{t}(x) \psi_{t}(x) n_{p}(t, d x)-r\left(t, q_{t}, \psi_{t}\right)\right] d t, \quad Q \text {-a.s. }
$$

By the definition of $g$, this implies $d A_{t} \leq\left[g_{1}\left(t, Z_{t}\right)+\int_{\mathbb{R} \backslash\{0\}} g_{2}\left(t, x, \tilde{Z}_{t}(x)\right) n_{p}(t, d x)\right] d t, Q$-a.s. Together with (A.27) we obtain that

$$
d A_{t}=\left[g_{1}\left(t, Z_{t}\right)+\int_{\mathbb{R} \backslash\{0\}} g_{2}\left(t, x, \tilde{Z}_{t}(x)\right) n_{p}(t, d x)\right] d t,
$$

$Q$-a.s. By Proposition A.1, (A.28)-(A.29) entail that $q_{t} \in \partial g_{1}\left(t, Z_{t}\right)$ and $\psi_{t}(x) \in \partial g_{2}\left(t, x, \tilde{Z}_{t}(x)\right)$, $d Q \times d t$ a.s. By Lemma A.19, it follows that $Q$ is an equivalent probability measure. Hence, the last equality holds $P$-a.s. Consequently, by (A.9), (A.11) and (A.29), $U_{t}(F)$ is indeed a solution to the BSDE (4.4). That $U_{t}(F)$ is the unique solution follows from Lemma A.22 below. This completes the proof.

Denote $C_{e}=\left\{Q \sim P \mid \mathrm{E}_{Q}\left[\int_{0}^{T}\left[r_{1}\left(s, q_{s}\right)+\int_{\mathbb{R} \backslash\{0\}} r_{2}\left(s, x, \psi_{s}(x)\right) n_{p}(s, d x)\right] d s\right]<\infty\right\}$.

Lemma A.22 Let $\left(Y^{\prime}, Z^{\prime}, \tilde{Z}^{\prime}\right)$ be a solution to a BSDE with driver functions $g_{1}$ and $g_{2}$ satisfying properties (b)-(c) above such that $g_{1}\left(s, Z_{s}^{\prime}\right)+\int_{\mathbb{R} \backslash\{0\}} g_{2}\left(s, x, \tilde{Z}_{s}^{\prime}(x)\right) n_{p}(s, d x)$ is uniformly bounded from below. (This is in particular the case if $g_{1}$ and $g_{2}$ are both non-negative.) Then 
we have that $Y_{t}^{\prime}=U_{t}(F)$, where $U(F)$ is given by (A.8) with $r_{1}$ and $r_{2}$ being the dual conjugates of $g_{1}$ and $g_{2}$. Furthermore,

$$
U_{t}(F)=\min _{Q \in C_{e}} \mathrm{E}_{Q}\left[F+\int_{t}^{T}\left[r_{1}\left(s, q_{s}\right)+\int_{\mathbb{R} \backslash\{0\}} r_{2}\left(s, x, \psi_{s}(x)\right) n_{p}(s, d x)\right] d s \mid \mathcal{F}_{t}\right] .
$$

Proof. Define $C=\left\{Q \ll P \mid \mathrm{E}_{Q}\left[\int_{0}^{T}\left[r_{1}\left(s, q_{s}\right)+\int_{\mathbb{R} \backslash\{0\}} r_{2}\left(s, x, \psi_{s}(x)\right) n_{p}(s, d x)\right] d s\right]<\infty\right\}$. Let $Q \in C$. We write

$$
\begin{aligned}
Y_{t}^{\prime}= & \mathrm{E}_{Q}\left[F-\int_{t}^{T}\left[g_{1}\left(s, Z_{s}^{\prime}\right)+\int_{\mathbb{R} \backslash\{0\}} g_{2}\left(s, x, \tilde{Z}_{s}^{\prime}(x)\right) n_{p}(s, d x)\right] d s\right. \\
& \left.+\int_{t}^{T} Z_{s}^{\prime} d W_{s}+\int_{t}^{T} \int_{\mathbb{R} \backslash\{0\}} \tilde{Z}_{s}^{\prime}(x) \tilde{N}_{p}(d s, d x) \mid \mathcal{F}_{t}\right] \\
= & \mathrm{E}_{Q}\left[F+\int_{t}^{T}\left[q_{s} Z_{s}^{\prime}-g_{1}\left(s, Z_{s}^{\prime}\right)+\int_{\mathbb{R} \backslash\{0\}}\left\{\tilde{Z}_{s}^{\prime}(x) \psi_{s}(x)-g_{2}\left(s, x, \tilde{Z}_{s}^{\prime}(x)\right)\right\} n_{p}(s, d x)\right] d s\right. \\
& \left.\quad+\int_{t}^{T} Z_{s}^{\prime} d W_{s}^{Q}+\int_{t}^{T} \int_{\mathbb{R} \backslash\{0\}} \tilde{Z}_{s}^{\prime}(x) \tilde{N}_{p}^{Q}(d s, d x) \mid \mathcal{F}_{t}\right] \\
= & \mathrm{E}_{Q}\left[F+\int_{t}^{T}\left[q_{s} Z_{s}^{\prime}-g_{1}\left(s, Z_{s}^{\prime}\right)+\int_{\mathbb{R} \backslash\{0\}}\left\{\tilde{Z}_{s}^{\prime}(x) \psi_{s}(x)-g_{2}\left(s, x, \tilde{Z}_{s}^{\prime}(x)\right)\right\} n_{p}(s, d x)\right] d s \mid \mathcal{F}_{t}\right] \\
\leq & \mathrm{E}_{Q}\left[F+\int_{t}^{T}\left[r_{1}\left(s, q_{s}\right)+\int_{\mathbb{R} \backslash\{0\}} r_{2}\left(s, x, \psi_{s}(x)\right) n_{p}(s, d x)\right] d s \mid \mathcal{F}_{t}\right],
\end{aligned}
$$

where we used in the first equality that $Y_{t}^{\prime}$ is $\mathcal{F}_{t}$-measurable. Note that the conditional expectation in the first equality is well-defined since $Y^{\prime}$ is bounded by the definition of a solution to a BSDE. The second and third equalities hold as $\int_{0}^{t} Z_{s} d W_{s}^{Q}$ and $\int_{0}^{t} \int_{\mathbb{R} \backslash\{0\}} \tilde{Z}_{s}^{\prime}(x) \tilde{N}_{p}^{Q}(d s, d x)$ are well-defined martingales. This may be seen since, by Lemma A.14 and Remark A.15 (with $J=Y^{\prime}$ and $\left.d A_{t}=\left[g_{1}\left(t, Z_{t}\right)+\int_{\mathbb{R} \backslash\{0\}} g_{2}\left(t, x, \tilde{Z}_{t}(x)\right) n_{p}(t, d x)\right] d t\right)$, we have that $Z^{\prime}$ and $\tilde{Z}^{\prime}$ are in $L^{2}(d Q \times d s)$ and $L^{2}\left(d Q \times n_{p}^{Q}(s, d x) \times d s\right)$, respectively.

It follows from (A.31) and the fact that we can restrict the essential infimum in (A.8) to $Q \in C$, that

$$
Y_{t}^{\prime} \leq U_{t}(F)
$$

Next, note that from Lemma A.14 and Remark A.15 it also follows that $Z^{\prime}$ is a $\mathrm{BMO}(P)$ process and $\tilde{Z}^{\prime}$ is $\operatorname{BMO}(P)$ function. By Lemma A.31, we may now choose a predictable $q_{s}^{*} \in \partial g_{1}\left(s, Z_{s}^{\prime}\right)$. Moreover, set $\psi_{s}^{*}(x) \in g_{2,+}^{\prime}\left(s, x, \tilde{Z}_{s}^{\prime}(x)\right) . q^{*}$ and $\psi^{*}$ induce a stochastic exponential martingale $M_{t}:=\mathcal{E}\left(\left(q^{*} \cdot W\right)_{t}+\left(\psi^{*} \cdot \tilde{N}_{p}\right)_{t}\right)$. By Lemma A.20, $\frac{d Q^{*}}{d P}:=M_{T}$ is an equivalent probability measure. Proceeding as in (A.31) with $q^{*}, \psi^{*}$ and $Q^{*}$ (where the inequality in (A.31) becomes an equality) yields

$$
Y_{t}^{\prime}=\mathrm{E}_{Q^{*}}\left[F-\int_{t}^{T}\left[r_{1}\left(s, q_{s}^{*}\right)+\int_{\mathbb{R} \backslash\{0\}} r_{2}\left(s, x, \psi_{s}^{*}(x)\right) n_{p}(s, d x)\right] d s \mid \mathcal{F}_{t}\right] .
$$

Thus, by the definition of $U_{t}(F)$ in (A.8), we get $Y_{t}^{\prime} \geq U_{t}(F)$. Therefore, indeed $Y^{\prime}=U(F)$. As the essential infimum in (A.8) is always attained in a $Q^{*}$ equivalent to $P,($ A.30) also follows. 
Remark A.23 Theorem A.21 assumes that $g_{1}$ and $g_{2}$ satisfy (a)-(e). However, assumption (b) may be replaced by assumption ( $\left.\mathrm{b}^{\prime}\right)$. This is seen as follows: As for a bounded terminal condition $F$, the corresponding $\tilde{Z}$ is bounded by $2\|F\|_{\infty}$, it is sufficient that property (b) holds for $\tilde{z}$ bounded by an arbitrary constant. (Of course, (a) and (c)-(e) must still hold.) The

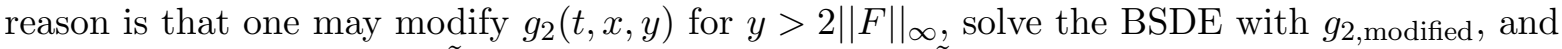
then observe that, since $|\tilde{Z}| \leq 2\|F\|_{\infty}, g_{2 \text {,modified }}\left(t, x, \tilde{Z}_{t}(x)\right)$ agrees with the original driver $g_{2}\left(t, x, \tilde{Z}_{t}(x)\right)$. Therefore, it is sufficient that $g_{2}$ satisfies property (b) for $\tilde{z}$ bounded by a prespecified constant coming from the terminal condition. This is equivalent to that, for every $\bar{C}>0$, there exists a bounded functional $\tilde{A} \in L^{2, \infty}$ and $K^{\prime \prime}>0$, such that for every $a$ bounded by $\bar{C}$ we have

$$
g_{2}(t, x, a) \leq \tilde{A}_{t}(x)+K^{\prime \prime} a^{2} .
$$

Proof of Theorem 4.1. Theorem 4.1 follows from Lemma A.7 and Theorem A.21.

We now prepare the proof of Theorem 4.2.

Theorem A.24 Suppose that $g_{1}$ and $g_{2}$ satisfy (a)-(e). Let $A_{s}$ and $B_{s}$ be predictable and bounded processes, and let $\tilde{H}_{s}(x)$ and $\tilde{C}_{s}(x)$ be predictable and bounded functionals in $L^{2, \infty}$ (see Section 2 for the definition). Then every BSDE with bounded terminal condition $F$ and driver function

$$
g(t, z, \tilde{z}):=B_{t}+g_{1}\left(t, z-A_{t}\right)+\int_{\mathbb{R} \backslash\{0\}}\left[\tilde{H}_{t}(x)+g_{2}\left(t, x, \tilde{z}(x)-\tilde{C}_{t}(x)\right)\right] n_{p}(t, d x)
$$

has a unique solution $(Y, Z, \tilde{Z})$. Moreover, $Z$ is a $B M O(P)$ process, $\tilde{Z}$ is $B M O(P)$ function, and we have

$$
\begin{aligned}
Y_{t}=\min _{Q \in C_{e}} \mathrm{E}_{Q}[F & +\int_{t}^{T}\left[-B_{s}+A_{s} q_{s}+r_{1}\left(s, q_{s}\right)\right. \\
& \left.\left.+\int_{\mathbb{R} \backslash\{0\}}\left\{\psi_{s}(x) \tilde{C}_{s}(x)-\tilde{H}_{s}(x)+r_{2}\left(s, x, \psi_{s}(x)\right)\right\} n_{p}(s, d x)\right] d s \mid \mathcal{F}_{t}\right] .
\end{aligned}
$$

Proof. Define

$\hat{g}_{1}(t, z):=g_{1}\left(t, z-A_{t}\right)-g_{1}\left(t,-A_{t}\right)$ and $\hat{g}_{2}(t, x, \tilde{z}(x)):=g_{2}\left(t, x, \tilde{z}(x)-\tilde{C}_{t}(x)\right)-g_{2}\left(t, x,-\tilde{C}_{t}(x)\right)$.

Now clearly, $\hat{g}_{1}$ and $\hat{g}_{2}$ satisfy property (c). That $\hat{g}_{2}$ satisfies property (b) may be seen as

$$
\begin{aligned}
\hat{g}_{2}(t, \omega, x, \tilde{z}(x)) & \leq g_{2}\left(t, \omega, x, \tilde{z}(x)-\tilde{C}_{t}(\omega, x)\right)+\left|g_{2}\left(t, \omega, x,-\tilde{C}_{t}(\omega, x)\right)\right| \\
& \leq K_{2} \Phi\left(\frac{\tilde{z}(x)-\tilde{C}_{t}(\omega, x)}{K_{2}}\right)+2 \tilde{A}_{t}(\omega, x)+K_{2} \Phi\left(\frac{-\tilde{C}_{t}(\omega, x)}{K_{2}}\right) \\
& =K_{2} \Phi\left(\frac{1}{2}\left(\frac{2 \tilde{z}(x)}{K_{2}}+\frac{-2 \tilde{C}_{t}(\omega, x)}{K_{2}}\right)\right)+2 \tilde{A}_{t}(\omega, x)+K_{2} \Phi\left(\frac{-\tilde{C}_{t}(\omega, x)}{K_{2}}\right) \\
& \leq \frac{K_{2}}{2} \Phi\left(\frac{2 \tilde{z}(x)}{K_{2}}\right)+\frac{K_{2}}{2} \Phi\left(\frac{-2 \tilde{C}_{t}(\omega, x)}{K_{2}}\right)+K_{2} \Phi\left(\frac{-\tilde{C}_{t}(\omega, x)}{K_{2}}\right)+2 \tilde{A}_{t}(\omega, x) \\
& =\frac{K_{2}}{2} \Phi\left(\frac{2 \tilde{z}(x)}{K_{2}}\right)+\tilde{B}_{t}(\omega, x),
\end{aligned}
$$


where the bounded functional $\tilde{B}_{t}(x):=\frac{K_{2}}{2} \Phi\left(\frac{-2 \tilde{C}_{t}(x)}{K_{2}}\right)+K_{2} \Phi\left(\frac{-\tilde{C}_{t}(x)}{K_{2}}\right)+2 \tilde{A}_{t}(x)$ by Corollary A.6 is in $L^{2, \infty}$. In the first inequality, we applied the definition of $\hat{g}_{2}$. In the second inequality, we used that $g_{2}$ satisfies property (b). In the third inequality, we used that $\Phi$ is convex. By (A.35), $\hat{g}_{2}$ satisfies property (b). That $\hat{g}_{1}$ satisfies (b) is straightforward to see. Now it is also not hard to show that $\hat{g}_{1}$ satisfies property (d) and $\hat{g}_{2}$ satisfies property (e). Hence, $\hat{g}_{1}$ and $\hat{g}_{2}$ satisfy (b)-(e). Unfortunately, $\hat{g}_{1}$ and $\hat{g}_{2}$ do not satisfy (a). Therefore, we have to define new functions $\hat{g}_{1}^{P^{*}}$ and $\hat{g}_{2}^{P^{*}}$.

For this purpose, set $\psi_{s}^{*}(x):=g_{2,+}^{\prime}\left(s, x,-\tilde{C}_{s}(x)\right)$. By property (e) and the fact that $\tilde{C}$ is bounded and in $L^{2, \infty}$, we have that (i) $\psi^{*} \in L^{2, \infty}$, (ii) $\psi^{*}$ is bounded by a constant, say $\bar{C}$, and (iii) $\psi^{*} \geq-1+\epsilon$. In particular, $\psi^{*}$ is a $\operatorname{BMO}(P)$ function. Next, choose a predictable bounded process $q^{*}$ such that $q_{s}^{*} \in \partial g_{1}\left(s,-A_{s}\right)$. Define a new reference measure $P^{*}$ by setting $\frac{d P^{*}}{d P}=\mathcal{E}\left(\left(q^{*} \cdot W\right)_{T}+\left(\psi^{*} \cdot \tilde{N}_{p}\right)_{T}\right)$. Note that as $q^{*}$ is bounded and $\psi^{*}$ is $\operatorname{BMO}(P)$ function, $P^{*}$ is a well-defined probability measure. Next, define driver functions

$$
g_{1}^{P^{*}}(t, z):=-z q_{t}^{*}+\hat{g}_{1}(t, z) \quad \text { and } \quad g_{2}^{P^{*}}(t, x, a):=\frac{1}{1+\psi_{t}^{*}(x)}\left(-\psi_{t}^{*}(x) a+\hat{g}_{2}(t, x, a)\right) .
$$

Set $g^{P^{*}}(t, z, \tilde{z})=g_{1}^{P^{*}}(t, z)+\int_{\mathbb{R} \backslash\{0\}} g_{2}^{P^{*}}(t, x, \tilde{z}(x)) n_{p}^{P^{*}}(t, d x)$. By the definition of $q^{*}$ and $\psi^{*}$ we have that $g_{1}^{P^{*}}$ and $g_{2}^{P^{*}}$ have their minimum at $z=0$ and $a=0$, respectively. Furthermore, these minima are both equal to zero. Hence, $g_{1}^{*}$ and $g_{2}^{*}$ satisfy property (a). To see property (b) for $g_{1}^{P^{*}}$, note that

$$
\left|g_{1}^{P^{*}}(t, \omega, z)\right| \leq \frac{1}{2}|z|^{2}+\frac{1}{2}\left|q_{t}^{*}(\omega)\right|^{2}+\bar{K}\left(1+|z|^{2}\right) \leq K^{\prime \prime}\left(1+|z|^{2}\right),
$$

for a $K^{\prime \prime}>0$. Note that we have used in the last inequality that $q^{*}$ is uniformly bounded. To see property (b) for $g_{2}^{P^{*}}$, notice that by Remark A.23 it is sufficient to show (A.32). So let $a$ be bounded by a constant, say $C$. Then by (A.35) and the fact that $\psi^{*} \geq-1+\epsilon$,

$$
\begin{aligned}
\left|g_{2}^{P^{*}}(t, \omega, x, a)\right| & \leq \frac{1}{\epsilon}\left(\Psi\left(\psi_{t}^{*}(\omega, x)\right)+\Phi(-a)+\tilde{B}_{t}(\omega, x)+\frac{K_{2}}{2} \Phi\left(2 a / K_{2}\right)\right) \\
& \leq \frac{1}{\epsilon}\left(\Psi\left(\psi_{t}^{*}(\omega, x)\right)+\left(e^{C}+2 \frac{e^{(2 C) / K_{2}}}{K_{2}}\right) a^{2}+\tilde{B}_{t}(\omega, x)\right),
\end{aligned}
$$

where we used (A.1)-(A.2). Since $0 \leq \Psi(x) \leq x^{2}$ for all $x \geq-1$ and as $\psi^{*}$ is bounded by $\bar{C}$ we have that $\Psi\left(\psi^{*}\right) \leq\left|\psi^{*}\right|^{2} \leq \bar{C}\left|\psi^{*}\right| \in L^{2, \infty}$. Therefore, the bounded functional $\Psi\left(\psi^{*}\right)$ is in $L^{2, \infty}$ and we get that $\hat{g}_{2}^{P^{*}}$ satisfies (A.32). That $\hat{g}_{1}^{P^{*}}$ and $\hat{g}_{1}^{P^{*}}$ also satisfy (d)-(e) is seen similarly.

Therefore, by Theorem A.21 and Remark A.23, we may define $(\hat{Y}, Z, \tilde{Z})$ as the unique solution to the BSDE

$$
\begin{aligned}
d \hat{Y}_{s} & =g^{P^{*}}\left(s, Z_{s}, \tilde{Z}_{s}\right) d s-Z_{s} d W_{s}^{P^{*}}-\int_{\mathbb{R} \backslash\{0\}} \tilde{Z}_{s}(x) \tilde{N}_{p}^{P^{*}}(d s, d x) \\
\hat{Y}_{T} & =\hat{F}
\end{aligned}
$$


with $\hat{F}:=F-\int_{0}^{T}\left[B_{s}+g_{1}\left(s,-A_{s}\right)+\int_{\mathbb{R} \backslash\{0\}}\left\{\tilde{H}_{s}(x)+g_{2}\left(s, x,-\tilde{C}_{s}(x)\right)\right\} n_{p}(s, d x)\right] d s$. (Note that the terminal condition is bounded.) In particular,

$$
\begin{aligned}
d \hat{Y}_{s}= & {\left[-Z_{s} q_{s}^{*}+\hat{g}_{1}\left(s, Z_{s}\right)+\int_{\mathbb{R} \backslash\{0\}} \frac{-\psi_{s}^{*}(x) \tilde{Z}_{s}(x)+\hat{g}_{2}\left(s, x, \tilde{Z}_{s}(x)\right)}{1+\psi_{s}^{*}(x)} n_{p}^{P^{*}}(s, d x)\right] d s } \\
& -Z_{s} d W_{s}^{P^{*}}-\int_{\mathbb{R} \backslash\{0\}} \tilde{Z}_{s}(x) \tilde{N}_{p}^{P^{*}}(d s, d x) \\
= & {\left[\hat{g}_{1}\left(s, Z_{s}\right)+\int_{\mathbb{R} \backslash\{0\}} \hat{g}_{2}\left(s, x, \tilde{Z}_{s}(x)\right) n_{p}(s, d x)\right] d s-Z_{s} d W_{s}-\int_{\mathbb{R} \backslash\{0\}} \tilde{Z}_{s}(x) \tilde{N}_{p}(d s, d x) . }
\end{aligned}
$$

Therefore, under the measure $P$ we have that $Y$ is a solution to the BSDE with terminal condition $\hat{F}$ and driver function $\hat{g}(t, z, \tilde{z})=\hat{g}_{1}(t, z)+\int_{\mathbb{R} \backslash\{0\}} \hat{g}_{2}(t, x, \tilde{z}(x)) n_{p}(t, d x)$. As $\hat{g}$ is uniformly bounded from below, Lemma A.14 and Remark A.15 yield that $Z$ is a $\mathrm{BMO}(P)$ process and $\tilde{Z}$ is $\operatorname{BMO}(P)$ function. The transformation

$$
Y_{t}:=\hat{Y}_{t}+\int_{0}^{t}\left[B_{s}+g_{1}\left(s,-A_{s}\right)+\int_{\mathbb{R} \backslash\{0\}}\left\{\tilde{H}_{s}(x)+g_{2}\left(s, x,-\tilde{C}_{s}(x)\right)\right\} n_{p}(s, d x)\right] d s
$$

by the definition of $\hat{g}$ and $g$ (see (A.33)) yields the BSDE

$$
\begin{aligned}
d Y_{s} & =g\left(s, Z_{s}, \tilde{Z}_{s}\right) d s-Z_{s} d W_{s}-\int_{\mathbb{R} \backslash\{0\}} \tilde{Z}_{s}(x) \tilde{N}_{p}(d s, d x), \\
Y_{T} & =F .
\end{aligned}
$$

Hence, the BSDEs (A.36) and (A.38) are equivalent. Now since (A.36) has a unique solution, (A.38) has a unique solution as well.

Finally, to see (A.34), note that the dual conjugates of $\hat{g}_{1}$ and $\hat{g}_{2}$ are given by $\hat{r}_{1}(s, q)=$ $A_{s} q+r_{1}(s, q)+g_{1}\left(s,-A_{s}\right)$ and $\hat{r}_{2}(s, x, y)=y \tilde{C}_{s}(x)+r_{2}(s, x, y)+g_{2}\left(s, x,-\tilde{C}_{s}(x)\right)$. As $\hat{g}$ is uniformly bounded from below, by Lemma A.22 and (A.30) (with terminal condition $\hat{F}$ ), we have

$$
\begin{aligned}
\hat{Y}_{t}=\min _{Q \in C_{e}} \mathrm{E}_{Q}[F & -\int_{0}^{T}\left[B_{s}+g_{1}\left(s,-A_{s}\right)+\int_{\mathbb{R} \backslash\{0\}}\left\{\tilde{H}_{s}(x)+g_{2}\left(s, x,-\tilde{C}_{s}(x)\right)\right\} n_{p}(s, d x)\right] d s \\
& \left.+\int_{t}^{T}\left[\hat{r}_{1}\left(s, q_{s}\right)+\int_{\mathbb{R} \backslash\{0\}} \hat{r}_{2}\left(s, x, \psi_{s}(x)\right) n_{p}(s, d x)\right] d s \mid \mathcal{F}_{t}\right] .
\end{aligned}
$$

Together with (A.37), this yields (A.34).

Remark A.25 As for Theorem A.21 also for Theorem A.24 it is sufficient that $g_{1}$ and $g_{2}$ satisfy properties (a), (b'), (c)-(e).

We also call (A.34) the dual representation of the solution to the corresponding BSDE.

Proposition A.26 Suppose we have bounded terminal conditions $F$ and $G$, and driver functions $f(t, z, \tilde{z})$ and $g(t, z, \tilde{z})$ being of the form (A.33), respectively. Then the solutions of the corresponding BSDEs, say $Y^{1}$ and $Y^{2}$, satisfy a comparison principle, i.e., if $F \leq G$ and $f \geq g$ then $Y_{1} \leq Y_{2}$. 
Proof. Existence and uniqueness of $Y_{1}$ and $Y_{2}$ follow from Theorem A.24. The comparison principle follows directly from the dual representations.

Lemma A.27 We have that for every admissible $\pi, U_{t}\left(F+X_{T}^{(\pi)}\right)=U_{t}^{g^{(\pi)}}(F)+X_{t}^{(\pi)}$, where $U^{g^{(\pi)}}(F)$ is the unique solution to the BSDE with terminal condition $F$ and driver function $g^{(\pi)}(t, z, \tilde{z})=g_{1}^{(\pi)}(t, z)+\int_{\mathbb{R} \backslash\{0\}} g_{2}^{(\pi)}(t, x, \tilde{z}(x)) n_{p}(t, d x)$ with

$$
g_{1}^{(\pi)}(t, z, \tilde{z}):=g_{1}\left(t, z-\pi_{t} \sigma_{t}\right)-\pi_{t} b_{t} \quad \text { and } \quad g_{2}^{(\pi)}(t, x, \tilde{z}(x)):=g_{2}\left(t, x, \tilde{z}(x)-\pi_{t} \beta_{t}(x)\right) .
$$

Proof. By Theorem A.24 (with $A=\pi \sigma, B=-\pi b, \tilde{C}=\pi \beta$, and $\tilde{H}=0$ ), the BSDE

$$
\begin{aligned}
d \hat{Y}_{t}^{(\pi)} & =g^{(\pi)}\left(s, Z_{s}^{(\pi)}, \tilde{Z}_{s}^{(\pi)}\right) d s-Z_{s}^{(\pi)} d W_{s}-\int_{\mathbb{R} \backslash\{0\}} \tilde{Z}_{s}^{(\pi)}(x) \tilde{N}_{p}(d s, d x), \\
\hat{Y}_{T}^{(\pi)} & =F
\end{aligned}
$$

has a unique solution, which we denote by $U^{g^{(\pi)}}(F)$ with $\mathrm{BMO}(P)$ process $Z$ and $\operatorname{BMO}(P)$ function $\tilde{Z}$. Let $r_{1}^{(\pi)}$ and $r_{2}^{(\pi)}$ be the dual conjugates of $g_{1}^{(\pi)}$ and $g_{2}^{(\pi)}$ defined above, respectively. It is straightforward to verify that

$$
r_{1}^{(\pi)}(s, q)=\pi_{s} b_{s}+\pi_{s} \sigma_{s} q+r_{1}(s, q) \text { and } r_{2}^{(\pi)}(s, x, \psi(x))=\psi(x) \pi_{s} \beta_{s}(x)+r_{2}(s, x, \psi(x)) .
$$

(A.34) becomes then

$$
U^{g^{(\pi)}}(F)=\min _{Q \in C_{e}} \mathrm{E}_{Q}\left[F+\int_{t}^{T}\left[r_{1}^{(\pi)}\left(s, q_{s}\right)+\int_{\mathbb{R} \backslash\{0\}} r_{2}^{(\pi)}\left(s, x, \psi_{s}(x)\right) n_{p}(s, d x)\right] d s \mid \mathcal{F}_{t}\right] .
$$

As a result,

$$
\begin{aligned}
& U_{t}\left(F+X_{T}^{(\pi)}\right)-X_{t}^{(\pi)} \\
& =\min _{Q \in C_{e}} \mathrm{E}_{Q}\left[F+\int_{t}^{T} \pi_{s} b_{s}+\int_{t}^{T} \pi_{s} \sigma_{s} d W_{s}+\int_{t}^{T} \pi_{s} \beta_{s}(x) \tilde{N}_{p}(d s, d x)\right. \\
& \left.+\int_{t}^{T}\left[r_{1}\left(s, q_{s}\right)+\int_{\mathbb{R} \backslash\{0\}} r_{2}\left(s, x, \psi_{s}(x)\right) n_{p}(s, d x)\right] d s \mid \mathcal{F}_{t}\right] \\
& =\min _{Q \in C_{e}} \mathrm{E}_{Q}\left[F+\int_{t}^{T} \pi_{s} \sigma_{s} d W_{s}^{Q}+\int_{t}^{T} \pi_{s} \beta_{s}(x) \tilde{N}_{p}^{Q}(d s, d x)\right. \\
& \left.+\int_{t}^{T}\left[\pi_{s} b_{s}+\pi_{s} \sigma_{s} q_{s}+r_{1}\left(s, q_{s}\right)+\int_{\mathbb{R} \backslash\{0\}}\left\{\psi_{s}(x) \pi_{s} \beta_{s}(x)+r_{2}\left(s, x, \psi_{s}(x)\right)\right\} n_{p}(s, d x)\right] d s \mid \mathcal{F}_{t}\right] \\
& =\min _{Q \in C_{e}} \mathrm{E}_{Q}\left[F+\int_{t}^{T}\left[r_{1}^{(\pi)}\left(s, q_{s}\right)+\int_{\mathbb{R} \backslash\{0\}} r_{2}^{(\pi)}\left(s, x, \psi_{s}(x)\right) n_{p}(s, d x)\right] d s \mid \mathcal{F}_{t}\right] \\
& =U^{g^{(\pi)}}(F),
\end{aligned}
$$

where the first equality holds by (A.8). The third equality holds because $\int_{0}^{t} \pi_{s} \sigma_{s} d W_{s}^{Q}$ is a $Q$-martingale as $Q \sim P$ and $\pi$ and $\sigma$ are uniformly bounded. To see that also $\left((\pi \beta) \cdot \tilde{N}_{p}^{Q}\right)_{t}$ is 
a $Q$-martingale notice that by our assumptions $\pi_{s} \beta_{s}$ is uniformly bounded by a constant, say $C$. Thus,

$$
\begin{aligned}
\mathrm{E}_{Q}\left[\int_{0}^{T}\left(\pi_{s} \beta_{s}(x)\right)^{2} n_{p}^{Q}(s, d x) d s\right] & =\mathrm{E}_{Q}\left[\int_{0}^{T}\left(1+\psi_{s}(x)\right)\left(\pi_{s} \beta_{s}(x)\right)^{2} n_{p}(s, d x) d s\right] \\
& \leq K^{\prime \prime}+\mathrm{E}_{Q}\left[\int_{0}^{T} \psi_{s}(x)\left(\pi_{s} \beta_{s}(x)\right)^{2} n_{p}(s, d x) d s\right] \\
& \leq K^{\prime \prime}+\mathrm{E}_{Q}\left[\int_{0}^{T}\left[\Phi\left(\left(\pi_{s} \beta_{s}(x)\right)^{2}\right)+\Psi\left(\psi_{s}(x)\right)\right] n_{p}(s, d x) d s\right] \\
& \leq K^{\prime \prime}+\mathrm{E}_{Q}\left[\int_{0}^{T}\left[\Phi\left(C\left|\pi_{s} \beta_{s}(x)\right|\right)+\Psi\left(\psi_{s}(x)\right)\right] n_{p}(s, d x) d s\right] \\
& \leq K^{\prime \prime \prime}+\mathrm{E}_{Q}\left[\int_{0}^{T} \Psi\left(\psi_{s}(x)\right) n_{p}(s, d x) d s\right]<\infty,
\end{aligned}
$$

where we used that the components of $\beta$ are in $L^{2, \infty}$ in the first, and Corollary A.6 in the fourth inequality. The last term is smaller than infinity as $Q \in C_{e}$. It follows that $\left((\pi \beta) \cdot \tilde{N}_{p}^{Q}\right)_{t}$ is a $Q$-martingale. Now from (A.40) the lemma follows.

Denote $r^{(\pi)}\left(s, q_{s}, \psi_{s}(x)\right)=\int_{t}^{T} r_{1}^{(\pi)}\left(s, q_{s}\right)+\int_{\mathbb{R} \backslash\{0\}} r_{2}^{(\pi)}\left(s, x, \psi_{s}(x)\right) n_{p}(s, d x)$, where $r_{1}^{(\pi)}$ and $r_{2}^{(\pi)}$ are the dual conjugates of $g_{1}^{(\pi)}$ and $g_{2}^{(\pi)}$, respectively.

Proof of Theorem 4.2. By Lemma A.27,

$$
U_{t}\left(F+X_{T}^{(\pi)}\right)=U_{t}^{g^{(\pi)}}(F)+X_{t}^{(\pi)}
$$

where $U_{t}^{g^{(\pi)}}(F)$ is the unique solution to the BSDE with terminal condition $F$ and driver function $g^{(\pi)}$. Consequently,

$$
V_{0}(F)=\sup _{\pi \in \mathcal{A}} U_{0}\left(F+X_{T}^{(\pi)}\right)=\sup _{\pi \in \mathcal{A}}\left\{U_{0}^{g^{(\pi)}}(F)+X_{0}^{(\pi)}\right\}=\sup _{\pi \in \mathcal{A}} U_{0}^{g^{(\pi)}}(F)+w_{0} .
$$

Let $h(s, \pi):=-\pi b_{s}+g_{1}\left(s, Z_{s}-\pi \sigma_{s}\right)+\int_{\mathbb{R} \backslash\{0\}} g_{2}\left(s, x, \tilde{Z}_{s}(x)-\pi \beta_{s}(x)\right) n_{p}(s, d x)+J_{U}(\pi)$, where $J_{U}$ is infinity if $\pi \notin U$ and zero else. As $U$ is compact, $h^{*}$, the dual conjugate of $h$ with respect to $\pi$, is real-valued. Thus, by Lemma A.31, we can choose a predictable process $\pi^{*}$ such that $\pi_{s}^{*} \in \partial h^{*}(s, 0)$. By Proposition A.1, this implies that $0 \in \partial h\left(s, \pi_{s}^{*}\right)$. Therefore,

$$
\begin{aligned}
f(s, z, \tilde{z}) & =-\pi_{s}^{*} b_{s}+g\left(s, Z_{s}-\pi_{s}^{*} \sigma_{s}\right)+\int_{\mathbb{R} \backslash\{0\}} g_{2}\left(s, x, \tilde{Z}_{s}(x)-\pi_{s}^{*} \beta_{s}(x)\right) n_{p}(s, d x) \\
& =g^{\left(\pi^{*}\right)}\left(s, Z_{s}, \tilde{Z}_{s}\right),
\end{aligned}
$$

where $f$ was defined in (4.5). If we could show that

$$
\sup _{\pi \in \mathcal{A}} U_{0}^{g^{(\pi)}}(F)=Y_{0}
$$


then the theorem would follow from (A.42). Now ' $\geq$ ' in (A.44) is seen since $Y_{0}=U_{0}^{g^{\left(\pi^{*}\right)}}(F)$ by (A.43) and the definition of $Y$. On the other hand, ' $\leq$ ' follows as by (A.39) we have that

$$
\begin{aligned}
U_{0}^{g^{(\pi)}}(F) & =\min _{Q \in C_{e}} \mathrm{E}_{Q}\left[F+\int_{t}^{T} r^{(\pi)}\left(s, q_{s}, \psi_{s}\right) d s \mid \mathcal{F}_{t}\right] \\
& \leq \min _{Q \in C_{e}} \mathrm{E}_{Q}\left[F+\int_{t}^{T} r^{\left(\pi^{*}\right)}\left(s, q_{s}, \psi_{s}\right) d s \mid \mathcal{F}_{t}\right]=U_{0}^{g^{\left(\pi^{*}\right)}}(F)=Y_{0},
\end{aligned}
$$

where the inequality holds as $g^{(\pi)} \geq g^{\left(\pi^{*}\right)}$ so that for the dual conjugates we must have $r^{(\pi)} \leq$ $r^{\left(\pi^{*}\right)}$. Thus, we may infer that $Y_{0}+w_{0}$ is the optimal value. Since $Y_{0}+w_{0}=U_{0}^{g^{\left(\pi^{*}\right)}}(F)+w_{0}=$ $U_{0}\left(F+X_{T}^{\left(\pi^{*}\right)}\right), \pi^{*}$ is the optimal strategy.

From the proof of Theorem 4.2 we obtain the following Corollary.

Corollary A.28 Let $g$ be a driver with corresponding convex functions $g_{1}$ and $g_{2}$ satisfying (c). Define $g^{(\pi)}$ as in Lemma A.27. Suppose that for every strategy $(\pi)$, there exists a unique well-defined solution $U^{g^{(\pi)}}(F)$ of the BSDE (with driver $g^{(\pi)}$ and terminal condition $F$ ) which satisfies the dual representation. If then an evaluation $U\left(F+X_{T}^{(\pi)}\right)$ satisfies $(A .41)$, we have that the optimal value of the portfolio selection problem is given by $V_{0}(F)=Y_{0}+w_{0}$ for $Y$ given as the solution to the BSDE with terminal condition $F$ and driver function $f$ defined in (4.5). The optimal strategy is the one that attains the infimum in (4.5).

Proof of Theorem 4.3. To show Theorem 4.3, first some preliminaries: Choose a $Q \in M$. Then $Q \sim P$ and by the definition of $M$, one may see that we have not only that $H(Q \mid P)<\infty$ but also that $H(P \mid Q)<\infty$. Note further that

$$
X_{t}^{(\pi)}=w_{0}+\int_{0}^{t} \pi_{u}\left(\sigma_{u} d W_{u}^{Q}+b_{u}^{Q} d u\right)+\int_{0}^{t} \int_{\mathbb{R} \backslash\{0\}} \pi_{u} \beta_{u}(x) \tilde{N}_{p}^{Q}(d u, d x),
$$

where $b_{u}^{Q}=b_{u}+\sigma_{u} q_{u}+\int_{\mathbb{R} \backslash\{0\}} \psi_{u} \beta_{u}(x) n_{p}(u, d x)$. By our assumptions on $b, \sigma, \beta, q$ and $\psi, b^{Q}$ is uniformly bounded. (We can apply that $\left|\psi_{u} \beta_{u}(x)\right| \leq \frac{1}{2}\left|\psi_{u}\right|^{2}+\frac{1}{2}\left|\beta_{u}(x)\right|^{2}$ and that $Q \in M$.) Set $\hat{g}_{1}(z):=\frac{1}{2 \gamma}|z|^{2}, \hat{g}_{2}(\tilde{z}(x)):=\gamma \Phi\left(\frac{\tilde{z}(x)}{\gamma}\right)$, and $\hat{g}^{Q}(t, z, \tilde{z}(x)):=\hat{g}_{1}(z)+\int_{\mathbb{R} \backslash\{0\}} \hat{g}_{2}(\tilde{z}(x)) n_{p}^{Q}(t, d x)$. Furthermore, for $i=1,2$ define $\hat{g}_{i}^{Q,(\pi)}$ by

$$
\hat{g}_{1}^{Q,(\pi)}(t, z):=\hat{g}_{1}\left(t, z-\pi_{t} \sigma_{t}\right)-\pi_{t} b_{t}^{Q} \quad \text { and } \quad \hat{g}_{2}^{Q,(\pi)}(t, x, \tilde{z}(x)):=\hat{g}_{2}\left(t, x, \tilde{z}(x)-\pi_{t} \beta_{t}(x)\right) .
$$

Finally, set $\hat{g}^{Q,(\pi)}(t, z, \tilde{z})=\hat{g}_{1}^{Q,(\pi)}(t, z)+\int_{\mathbb{R} \backslash\{0\}} \hat{g}_{2}^{Q,(\pi)}(t, x, \tilde{z}(x)) n_{p}^{Q}(t, d x)$.

By Theorem A.24 (with the reference measure $P$ replaced by $Q$ ), the BSDE with driver function $\hat{g}^{Q,(\pi)}$ and terminal condition $F$ has a unique solution satisfying

$$
\begin{aligned}
d U_{s}^{\hat{g}^{Q,(\pi)}, Q}(F) & =\hat{g}^{Q,(\pi)}\left(s, Z_{s}^{\prime}, \tilde{Z}_{s}^{\prime}\right) d s-Z_{s}^{\prime} d W_{s}^{Q}-\int_{\mathbb{R} \backslash\{0\}} \tilde{Z}_{s}^{\prime}(x) \tilde{N}_{p}^{Q}(d s, d x), \\
U_{T}^{\hat{g}^{Q,(\pi)}, Q}(F) & =F .
\end{aligned}
$$

As $H(P \mid Q)<\infty$, by Lemma A.14 and Remark A.15, we have that $Z^{\prime} \in L^{2}(d P \times d s)$ and $\tilde{Z}^{\prime} \in L^{2}\left(d P \times n_{p}(s, d x) \times d s\right)$. Define

$$
U_{t}^{Q}\left(F+X_{T}^{(\pi)}\right):=U_{t}^{\hat{g}^{Q,(\pi)}, Q}(F)+X_{t}^{(\pi)},
$$


and set $Z:=Z^{\prime}-\pi \sigma$, and $\tilde{Z}:=\tilde{Z}^{\prime}-\pi \beta$. Then clearly $Z$ is in $L^{2}(d P \times d s)$ and $\tilde{Z}$ is in $L^{2}\left(d P \times n_{p}(s, d x) \times d s\right)$. We write

$$
\begin{aligned}
d U_{s}^{Q}\left(F+X_{T}^{(\pi)}\right) & =\hat{g}^{Q}\left(s, Z_{s}, \tilde{Z}_{s}\right) d s-Z_{s} d W_{s}^{Q}-\int_{\mathbb{R} \backslash\{0\}} \tilde{Z}_{s}(x) \tilde{N}_{p}^{Q}(d s, d x), \\
U_{T}^{Q}\left(F+X_{T}^{(\pi)}\right) & =F+X_{T}^{(\pi)}
\end{aligned}
$$

where the first equality follows from the BSDE (A.45) and the definition of $\hat{g}^{Q,(\pi)}$. By Lemma A.29 below,

$$
U_{t}^{Q}\left(F+X_{T}^{(\pi)}\right)=-\gamma \log \left(\mathrm{E}_{Q}\left[\exp \left\{\frac{-F-X_{T}^{(\pi)}}{\gamma}\right\} \mid \mathcal{F}_{t}\right]\right) .
$$

In order to use comparison principles it is more useful to consider the driver functions $\hat{g}^{Q,(\pi)}$ and the corresponding BSDEs under the measure $P$ instead of $Q$. For this purpose, define

$$
\begin{aligned}
g_{1}^{Q,(\pi)}(t, z, \tilde{z}) & :=\frac{1}{2 \gamma}\left|z-\pi_{t} \sigma_{t}\right|^{2}+\left(z-\pi_{t} \sigma_{t}\right) q_{t}-\pi_{t} b_{t}, \\
g_{2}^{Q,(\pi)}(t, x, \tilde{z}(x)) & :=\gamma\left(\psi_{t}(x)\left(\exp \left\{\frac{\tilde{z}(x)-\pi_{t} \beta_{t}(x)}{\gamma}\right\}-1\right)+\Phi\left(\frac{\tilde{z}(x)-\pi_{t} \beta_{t}(x)}{\gamma}\right)\right), \\
g^{Q,(\pi)}(t, z, \tilde{z}) & :=g_{1}^{Q,(\pi)}(t, z)+\int_{\mathbb{R} \backslash\{0\}} g_{2}^{Q,(\pi)}(t, x, \tilde{z}(x)) n_{p}(t, d x) .
\end{aligned}
$$

In Lemma A.30 below we show that for every strategy $(\pi), U^{\hat{g}^{Q,(\pi)}, Q}(F)$, (the solution to the BSDE under the measure $Q$ with driver function $\hat{g}^{Q,(\pi)}$ and terminal condition $F$ ) is equal to the unique solution to the BSDE under the measure $P$ with driver function $g^{Q,(\pi)}$ and terminal condition $F$. Henceforth, we denote the latter by $U^{g^{Q,(\pi)}}(F)$. Furthermore, it is shown in Lemma A.30 that $U^{g^{Q,(\pi)}}(F)$ satisfies the dual representation.

Next, set $\psi_{s}^{*}(x):=\left(d_{t}^{+}(x) I_{\{\tilde{z}(x) \geq 0\}}+d_{t}^{-}(x) I_{\{\tilde{z}(x)<0\}}\right)$ and choose a predictable $q_{t}^{*} \in \bar{g}_{1}(t, z-$ $\left.\pi_{t} \sigma_{t}\right)$ with $\bar{g}_{1}$ defined in (4.8). As $q^{*}$ and $\psi^{*}$ are well integrable we may define a probability measure $Q^{*}$ by setting $\frac{d Q^{*}}{d P}:=\mathcal{E}\left(\left(q^{*} \cdot W\right)_{T}+\left(\psi^{*} \cdot \tilde{N}_{p}\right)_{T}\right)$. Denote

$$
\begin{aligned}
g^{(\pi)}(s, z, \tilde{z}):=\sup _{Q \in M} g^{Q,(\pi)}(s, z, \tilde{z}) \\
=\frac{1}{2 \gamma}\left|z-\pi_{s} \sigma_{s}\right|^{2}+\left(z-\pi_{s} \sigma_{s}\right) q_{s}^{*}-\pi_{s} b_{s} \\
\quad+\gamma \int_{\mathbb{R} \backslash\{0\}}\left\{\psi_{s}^{*}(x)\left(\exp \left\{\frac{\tilde{z}(x)-\pi_{s} \beta_{s}(x)}{\gamma}\right\}-1\right)+\Phi\left(\frac{\tilde{z}(x)-\pi_{s} \beta_{s}(x)}{\gamma}\right)\right\} n_{p}(s, d x) \\
=g^{Q^{*},(\pi)}(s, z, \tilde{z}) .
\end{aligned}
$$

In particular, for every strategy $(\pi)$ there exists a solution $U_{t}^{g^{(\pi)}}(F)=U_{t}^{g^{Q^{*},(\pi)}}(F)$ to the BSDE under the measure $P$ with driver function $g^{(\pi)}$ and terminal condition $F$. The theorem would follow from Corollary A.28 if we could show that (A.41) holds for the evaluation given by $U_{t}\left(F+X_{T}^{(\pi)}\right)=\inf _{Q \in M}-\gamma \log \left(\mathrm{E}_{Q}\left[\exp \left\{\frac{-F-X_{T}^{(\pi)}}{\gamma}\right\} \mid \mathcal{F}_{t}\right]\right)$. To see this, first note that

$$
U_{t}^{g^{(\pi)}}(F)=\inf _{Q \in M} U_{t}^{g^{Q,(\pi)}}(F),
$$


where ' $\geq$ ' holds as $U_{t}^{g^{(\pi)}}(F)=U_{t}^{g^{Q^{*},(\pi)}}(F)$ (with $Q^{*}$ defined as above), while ' $\leq$ ' follows from the dual representations of $U_{t}^{g^{Q,(\pi)}}(F)$ shown in Lemma A.30(ii). (Note that by definition $g^{(\pi)} \geq g^{Q,(\pi)}$ for every $Q \in M$. For the conjugates of the driver in the dual representation this inequality is reversed.)

Therefore, we obtain that for every terminal payoff $F$,

$$
\begin{aligned}
U_{t}^{g^{(\pi)}}(F)+X_{t}^{(\pi)} & =\inf _{Q \in M} U_{t}^{g^{Q,(\pi)}}(F)+X_{t}^{(\pi)} \\
& =\inf _{Q \in M} U_{t}^{\hat{g}^{Q,(\pi)}, Q}(F)+X_{t}^{(\pi)} \\
& =\inf _{Q \in M} U_{t}^{Q}\left(F+X_{T}^{(\pi)}\right)=\inf _{Q \in M}-\gamma \log \left(\mathrm{E}_{Q}\left[\exp \left\{\frac{-F-X_{T}^{(\pi)}}{\gamma}\right\} \mid \mathcal{F}_{t}\right]\right),
\end{aligned}
$$

where the first equality holds by (A.52). The second equality holds by Lemma A.30(i). The third equality holds by (A.46), and the fourth equality holds by (A.48). Hence, the evaluation $U_{t}\left(F+X_{T}^{(\pi)}\right)=\inf _{Q \in M}-\gamma \log \left(\mathrm{E}_{Q}\left[\exp \left\{\frac{-F-X_{T}^{(\pi)}}{\gamma}\right\} \mid \mathcal{F}_{t}\right]\right)$ indeed satisfies (A.41). The theorem follows now from Corollary A.28.

Lemma A.29 (A.48) holds.

Proof. Set $M_{t}^{Q}:=\exp \left(-\frac{U_{t}^{Q}\left(F+X_{T}^{(\pi)}\right)}{\gamma}\right)$. By Itô's generalized formula and (A.47), we have

$$
\begin{aligned}
d M_{s}^{Q}= & M_{s-}^{Q}\left(\left[-\frac{\hat{g}^{Q}\left(s, Z_{s}, \tilde{Z}_{s}\right)}{\gamma}+\frac{1}{2 \gamma^{2}}\left|Z_{s}\right|^{2}\right] d s+\frac{Z_{s}}{\gamma} d W_{s}^{Q}+\int_{\mathbb{R} \backslash\{0\}} \frac{\tilde{Z}_{s}(x)}{\gamma} \tilde{N}_{p}^{Q}(d s, d x)\right. \\
& \left.\quad+\int_{\mathbb{R} \backslash\{0\}} \Phi\left(\frac{\tilde{Z}_{s}(x)}{\gamma}\right) N_{p}(d s, d x)\right) \\
= & M_{s-}^{Q}\left(\frac{Z_{s}}{\gamma} d W_{s}^{Q}+\int_{\mathbb{R} \backslash\{0\}}\left[\exp \left\{\tilde{Z}_{s}(x) / \gamma\right\}-1\right] \tilde{N}_{p}^{Q}(d s, d x)\right) .
\end{aligned}
$$

The second equality holds by the definition of $\hat{g}^{Q}$. Therefore, $M_{t}^{Q}$ is a local martingale given by $M_{t}^{Q}=M_{0} \mathcal{E}\left(\left(\frac{Z}{\gamma} \cdot W^{Q}\right)_{t}+\left([\exp \{\tilde{Z} / \gamma\}-1] \cdot \tilde{N}_{p}^{Q}\right)_{t}\right)$. Now it may be seen as in Morlais [58] that, for every $\gamma>0$, the set $\left\{\exp \left\{\frac{-X_{\sigma}^{(\pi)}}{\gamma}\right\} \mid \sigma\right.$ stopping time $\}$, is uniformly integrable. As $U^{\hat{g}^{Q,(\pi)}, Q}(F)$ is bounded and as by (A.46) $M_{t}^{Q}=\exp \left(-\frac{U_{t}^{\hat{g}^{Q,(\pi)}, Q}(F)+X_{t}^{(\pi)}}{\gamma}\right)$, we obtain that the set $\left\{M_{\sigma}^{Q} \mid \sigma\right.$ stopping time $\}$ is uniformly integrable. Thus, $M^{Q}$ is not only a local but also a true martingale. As, by definition, $M_{T}^{Q}=\exp \left(-\frac{F+X_{T}^{(\pi)}}{\gamma}\right)$, we get that $M_{t}^{Q}=$ $\mathrm{E}_{Q}\left[\exp \left\{-\frac{F+X_{T}^{(\pi)}}{\gamma}\right\} \mid \mathcal{F}_{t}\right]$. Now by the definition of $M^{Q}$, (A.48) follows.

Lemma A.30 For every $Q \in M$ the following holds:

(i) There exists a unique solution $U^{g^{Q,(\pi)}}(F)$ of the BSDE under the measure $P$ with driver $g^{Q,(\pi)}$ and terminal condition F. Furthermore, $U^{g^{Q,(\pi)}}(F)=U^{\hat{g}^{Q,(\pi)}, Q}(F)$ where $U^{\hat{g}^{Q,(\pi)}, Q}(F)$ was defined as the solution to the BSDE (A.45). 
(ii) $U^{g^{Q,(\pi)}}(F)$ satisfies a dual representation with respect to the reference measure $P$. (That means that $(q, \psi)$ are calculated with respect to $P$.)

Proof. (i): Uniqueness of a solution follows from (ii). On the other hand, from (A.45) it follows that $U_{s}^{\hat{g}^{Q,(\pi)}, Q}(F)$ satisfies

$$
\begin{aligned}
d U_{s}^{\hat{g}^{Q,(\pi)}, Q}(F) & =g^{Q,(\pi)}\left(s, Z_{s}^{\prime}, \tilde{Z}_{s}^{\prime}\right) d s-Z_{s}^{\prime} d W_{s}-\int_{\mathbb{R} \backslash\{0\}} \tilde{Z}_{s}^{\prime}(x) \tilde{N}_{p}(d s, d x), \\
U_{T}^{\hat{g}^{Q,(\pi)}, Q}(F) & =F
\end{aligned}
$$

with $g^{Q,(\pi)}$ defined in (A.51). Note that in the definition of $g^{Q,(\pi)}$ we switch from $b^{Q}$ to $b$, and from $n_{p}^{Q}$ to $n_{p}$. Since the existence of $U^{\hat{g}^{Q,(\pi)}, Q}(F)$ has already been shown, we have that $U^{g^{Q,(\pi)}}(F)$ the solution to a BSDE under the reference measure $P$ with terminal condition $F$ and driver function $g^{Q,(\pi)}$, is indeed equal to $U^{\hat{g}^{Q,(\pi)}, Q}(F)$. This proves (i).

(ii): If we could show that $g^{Q,(\pi)}\left(t, Z_{t}, \tilde{Z}_{t}^{\prime}\right)$ is bounded from below then it follows from (A.53) and Lemma A.22 that for every admissible strategy $\pi, U_{t}^{g^{Q,(\pi)}}(F)$ satisfies a dual representation (under $P$ ) with penalty function given by the dual conjugates of $g_{1}^{Q,(\pi)}$ and $g_{2}^{Q,(\pi)}$ defined in (A.49)-(A.50).

To see boundedness from below note that clearly for any $C>0$ clearly $a b \geq-\frac{a^{2}}{C^{2}}-C^{2} b^{2}$. Since $q$ is uniformly bounded, using this inequality on $\left(z-\pi_{s} \sigma_{s}\right) q_{s}$ with $C=\sqrt{2 \gamma}$ we can conclude that $g_{1}^{Q,(\pi)}$ is uniformly bounded from below. On the other hand by (A.45), $\tilde{Z}^{\prime}$ has to be bounded uniformly by $2\|F\|_{\infty}$. Therefore, $\tilde{Z}^{\prime}-\pi \beta$ is uniformly bounded by another constant say $C^{\prime}$. Hence, for any $C>0$,

$$
\begin{aligned}
& \frac{g_{2}^{Q,(\pi)}\left(t, x, \tilde{Z}_{t}^{\prime}(x)\right)}{\gamma}=\psi_{t}(x)\left(\exp \left\{\frac{\tilde{Z}_{t}^{\prime}(x)-\pi_{t} \beta_{t}(x)}{\gamma}\right\}-1\right)+\Phi\left(\frac{\tilde{Z}_{t}^{\prime}(x)-\pi_{t} \beta_{t}(x)}{\gamma}\right) \\
& \geq-C^{2} \psi_{t}^{2}(x)-\frac{1}{C^{2}}\left(\exp \left\{\frac{\tilde{Z}_{t}^{\prime}(x)-\pi_{t} \beta_{t}(x)}{\gamma}\right\}-1\right)^{2}+\frac{e^{-C^{\prime} / \gamma}}{2}\left(\frac{\tilde{Z}_{t}^{\prime}(x)-\pi_{t} \beta_{t}(x)}{\gamma}\right)^{2} \\
& \geq-C^{2} \psi_{t}^{2}(x)-\frac{e^{-2 C^{\prime} / \gamma}}{C^{2}}\left(\frac{\tilde{Z}_{t}^{\prime}(x)-\pi_{t} \beta_{t}(x)}{\gamma}\right)^{2}+\frac{e^{-C^{\prime} / \gamma}}{2}\left(\frac{\tilde{Z}_{t}^{\prime}(x)-\pi_{t} \beta_{t}(x)}{\gamma}\right)^{2},
\end{aligned}
$$

where we used (A.3) in the first and (A.4) in the second inequality. Hence, choosing $C$ large enough, it follows that there exists $B>0$ such that

$$
\frac{g_{2}^{Q,(\pi)}\left(t, x, \tilde{Z}_{t}^{\prime}(x)\right)}{\gamma} \geq-C^{2} \psi_{t}^{2}(x)+B\left(\frac{\tilde{Z}_{t}^{\prime}(x)-\pi_{t} \beta_{t}(x)}{\gamma}\right)^{2} .
$$

In particular, $g_{2}^{Q,(\pi)}\left(t, x, \tilde{Z}_{t}^{\prime}(x)\right)$ is bounded from below by $-C^{2} \psi_{t}^{2}(x)$. As $Q \in M$ so that $\psi$ is in $L^{2, \infty}$, we get that $\int_{\mathbb{R} \backslash\{0\}} g_{2}^{Q,(\pi)}\left(t, x, \tilde{Z}_{t}^{\prime}(x)\right) n_{p}(t, d x)$ is bounded from below. Together with $g_{1}^{Q,(\pi)}$ being bounded from below this implies that $g^{Q,(\pi)}\left(t, Z_{t}, \tilde{Z}_{t}\right)=g_{1}^{Q,(\pi)}\left(t, Z_{t}\right)+$ $\int_{\mathbb{R} \backslash\{0\}} g_{2}^{Q,(\pi)}\left(t, x, \tilde{Z}_{t}^{\prime}(x)\right) n_{p}(t, d x)$ is bounded from below. The finishes the proof of (ii). 
Proof of Theorem 4.5. As the components of $\beta$ are in $L^{2, \infty}$ and as by assumption $\rho \beta$ is bounded away from -1 , clearly $\log \left(1+\rho_{s} \beta_{s}(x)\right) \in L^{2, \infty}$ as well. Let

$$
\begin{aligned}
& g^{(\rho)}(s, z, \tilde{z})=g_{1}\left(s, z-\gamma \rho_{s} \sigma_{s}\right)-\gamma \rho_{s} b_{s}+\frac{\gamma}{2}\left|\rho_{s} \sigma_{s}\right|^{2} \\
& \quad+\int_{\mathbb{R} \backslash\{0\}}\left[-\gamma \log \left(1+\rho_{s} \beta_{s}(x)\right)+\gamma \rho_{s} \beta_{s}(x)+g_{2}\left(s, x, \tilde{z}(x)-\gamma \log \left(1+\rho_{s} \beta_{s}(x)\right)\right)\right] n_{p}(s, d x) .
\end{aligned}
$$

By Theorem A.24, there exists a unique solution, say $Y_{t}^{(\rho)}$, to the BSDE with terminal condition 0 and driver function $g^{(\rho)}$. It follows from (A.34) that

$$
Y_{t}^{(\rho)}=\min _{Q \in C_{e}} \mathrm{E}_{Q}\left[\int_{t}^{T}\left[r_{1}^{(\rho)}\left(s, q_{s}\right)+\int_{\mathbb{R} \backslash\{0\}} r_{2}^{(\rho)}\left(s, x, \psi_{s}(x)\right) n_{p}(s, d x)\right] d s \mid \mathcal{F}_{t}\right]
$$

with $r_{i}^{(\rho)}$ being the dual conjugate of $g_{i}^{(\rho)}$ for $i=1,2$, given by

$$
\begin{aligned}
r_{1}^{(\rho)}(s, q): & =\gamma\left[\rho_{s} b_{s}-\frac{1}{2}\left|\rho_{s} \sigma_{s}\right|^{2}+\rho_{s} \sigma_{s} q\right]+r_{1}(s, q), \\
r_{2}^{(\rho)}(s, x, \psi(x)): & =\gamma\left[\log \left(1+\rho_{s} \beta_{s}(x)\right)-\rho_{s} \beta_{s}(x)+\log \left(1+\rho_{s} \beta_{s}(x)\right) \psi(x)\right]+r_{2}(s, x, \psi(x)) .
\end{aligned}
$$

Furthermore, set $r^{(\rho)}(s, q, \psi):=r_{1}^{(\rho)}(s, q)+\int_{\mathbb{R} \backslash\{0\}} r_{2}^{(\rho)}(s, x, \psi(x)) n_{p}(s, d x)$. Using (4.10), we get

$$
\begin{aligned}
& U_{t}\left(\gamma \log \left(X_{T}^{(\rho)}\right)\right)-\gamma \log \left(X_{t}^{(\rho)}\right) \\
=\min _{Q \in C_{e}} & \mathrm{E}_{Q}\left[\int_{t}^{T}\left(\gamma \rho_{s} b_{s}-\frac{\gamma}{2}\left|\rho_{s} \sigma_{s}\right|^{2}\right) d s+\gamma \int_{t}^{T} \rho_{s} \sigma_{s} d W_{s}+\gamma \int_{t}^{T} \log \left(1+\rho_{s} \beta_{s}(x)\right) \tilde{N}_{p}(d s, d x)\right. \\
& +\gamma \int_{t}^{T} \int_{\mathbb{R} \backslash\{0\}}\left[\log \left(1+\rho_{s} \beta_{s}(x)\right)-\rho_{s} \beta_{s}(x)\right] n_{p}(s, d x) d s \\
& \left.\quad \int_{t}^{T}\left[r_{1}\left(s, q_{s}\right)+\int_{\mathbb{R} \backslash\{0\}} r_{2}\left(s, x, \psi_{s}(x)\right) n_{p}(s, d x)\right] d s \mid \mathcal{F}_{t}\right] \\
= & \min _{Q \in C_{e}} \mathrm{E}_{Q}\left[\gamma \int_{t}^{T} \rho_{s} \sigma_{s} d W_{s}^{Q}+\gamma \int_{t}^{T} \log \left(1+\rho_{s} \beta_{s}(x)\right) \tilde{N}_{p}^{Q}(d s, d x)+\int_{t}^{T}\left[\gamma \rho_{s} b_{s}-\frac{\gamma}{2}\left|\rho_{s} \sigma_{s}\right|^{2}\right.\right. \\
& +\gamma \rho_{s} \sigma_{s} q_{s}+r_{1}\left(s, q_{s}\right)+\int_{\mathbb{R} \backslash\{0\}}\left\{\gamma \log \left(1+\rho_{s} \beta_{s}(x)\right)-\gamma \rho_{s} \beta_{s}(x)\right. \\
& \left.\left.\left.+\gamma \log \left(1+\rho_{s} \beta_{s}(x)\right) \psi_{s}(x)+r_{2}\left(s, x, \psi_{s}(x)\right)\right\} n_{p}(s, d x)\right] d s \mid \mathcal{F}_{t}\right] \\
= & \min _{Q \in C_{e}} \mathrm{E}_{Q}\left[\int_{t}^{T}\left[r_{1}^{(\rho)}\left(s, q_{s}\right)+\int_{\mathbb{R} \backslash\{0\}} r_{2}^{(\rho)}\left(s, x, \psi_{s}(x)\right) n_{p}(s, d x)\right] d s \mid \mathcal{F}_{t}\right]=Y_{t}^{(\rho)} .
\end{aligned}
$$

The last equality holds by (A.54). Hence, $Y_{t}^{(\rho)}=U_{t}\left(\gamma \log \left(X_{T}^{(\rho))}\right)-\gamma \log \left(X_{t}^{(\rho)}\right)\right.$. This yields

$$
V_{0}=\sup _{\rho \in \mathcal{A}} U_{0}\left(\gamma \log \left(X_{T}^{(\rho))}\right)=\sup _{\rho \in \mathcal{A}}\left\{Y_{0}^{(\rho)}+\gamma \log \left(X_{0}^{(\rho)}\right)\right\}=\sup _{\rho \in \mathcal{A}} Y_{0}^{(\rho)}+\gamma \log \left(w_{0}\right) .\right.
$$


By Lemma A.31 below, we can choose a predictable $\rho^{*}$ such that

$$
\begin{aligned}
& f(s, z, \tilde{z})=g_{1}\left(s, z-\gamma \rho_{s}^{*} \sigma_{s}\right)-\gamma \rho_{s}^{*} b_{s}+\frac{\gamma}{2}\left|\rho_{s}^{*} \sigma_{s}\right|^{2} \\
& \quad+\int_{\mathbb{R} \backslash\{0\}}\left[-\gamma \log \left(1+\rho_{s}^{*} \beta_{s}(x)\right)+\gamma \rho_{s}^{*} \beta_{s}(x)+g_{2}\left(s, x, \tilde{z}(x)-\gamma \log \left(1+\rho_{s}^{*} \beta_{s}(x)\right)\right)\right] n_{p}(s, d x) \\
& \quad=g^{\left(\rho^{*}\right)}(s, z, \tilde{z}),
\end{aligned}
$$

where $f$ was defined in (4.12). By the definition of $Y$ in (4.13) this yields that $Y=Y^{\left(\rho^{*}\right)}$. If we could show that

$$
\sup _{\rho \in \mathcal{A}} Y_{0}^{(\rho)}=Y_{0}
$$

then from (A.55) the theorem would follow. Now ' $\geq$ ' in (A.56) follows as $Y_{0}=Y_{0}^{\left(\rho^{*}\right)}$. On the other hand ' $\leq$ ' follows since by (A.54) we have that

$$
Y_{0}^{(\rho)}=\min _{Q \in C_{e}} \mathrm{E}_{Q}\left[\int_{t}^{T} r^{(\rho)}\left(s, q_{s}, \psi_{s}\right) d s \mid \mathcal{F}_{t}\right] \leq \min _{Q \in C_{e}} \mathrm{E}_{Q}\left[\int_{t}^{T} r^{\left(\rho^{*}\right)}\left(s, q_{s}, \psi_{s}\right) d s \mid \mathcal{F}_{t}\right]=Y_{0}^{\left(\rho^{*}\right)}
$$

where the inequality holds as $g^{(\rho)} \geq g^{\left(\rho^{*}\right)}=f$ so that for the dual conjugates we must have $r^{(\rho)} \leq r^{\left(\rho^{*}\right)}$. Thus, we may conclude that $V_{0}=Y_{0}+\gamma \log \left(w_{0}\right)$. Since $Y_{0}+\gamma \log \left(w_{0}\right)=$ $U_{0}\left(\gamma \log \left(X_{T}^{\left(\rho^{*}\right)}\right)\right), \rho^{*}$ is the optimal strategy.

We finally need the following announced lemma, which is similar to Lemma 6.2 in Cheridito and Stadje [13].

Lemma A.31 Let $f:[0, T] \times \Omega \times \mathbb{R}^{1 \times d} \rightarrow \mathbb{R} \cup\{\infty\}$ be a function such that, for every $x, f(t, x)$ is a predictable process and

$$
f(t, \lambda x+(1-\lambda) y) \leq \lambda f(t, x)+(1-\lambda) f(t, y) \quad \text { for all } 0 \leq \lambda \leq 1 .
$$

Then, for every $x \in \mathbb{R}^{d}$ such that $\partial f(t, x) \neq \emptyset$, there exists $Z \in \mathcal{P}$ such that

$$
f(t, x+y)-f(t, x) \geq y Z_{t} \quad \text { for all } y \in \mathbb{R}^{d} .
$$

In particular, $Z_{t} \in \partial f(t, x)$, for all $t \in[0, T]$ a.s. Furthermore, if $X$ is a predictable process then it is also possible to choose $Z \in \mathcal{P}$ such that $Z_{t} \in \partial f(t, X)$, for all $t \in[0, T]$ a.s.

Proof. Let $x \in \mathbb{R}^{d}$ with $\partial f(t, x) \neq \emptyset$ and denote by $e_{1}, \ldots, e_{d}$ the canonical basis in $\mathbb{R}^{d}$. We construct $Z^{1}, \ldots, Z^{d} \in L^{0}(\mathcal{F})$ inductively. First define the mapping $p:[0, T] \times \Omega \times \mathbb{R}^{d} \rightarrow \mathcal{S}$ by

$$
p(t, y)=\inf _{n \in \mathbb{N}} n[f(t, x+y / n)-f(t, x)]
$$

and observe that

$$
\begin{aligned}
p(t, y) & \leq f(t, x+y)-f(t, x) \quad \text { for all } y \in \mathbb{R}^{d} \\
p(t, \lambda y) & =\lambda p(t, y) \text { for all } \lambda \geq 0 \text { and } y \in \mathbb{R}^{d} \\
p(t, y+z) & \leq p(t, y)+p(t, z) \quad \text { for all } y, z \in \mathbb{R}^{d}
\end{aligned}
$$


By (A.57) and (A.59), one has $p(t, y)<\infty$ and $p(t, y) \geq p(t, 0)-p(t,-y)>-\infty$ for all $y \in \mathbb{R}^{d}$. Furthermore, clearly $p$ is in $\mathcal{S}$ as infimum of predictable processes. So $p$ maps $[0, T] \times \Omega \times \mathbb{R}^{d}$ to $\mathcal{S}$. Now set $Z_{t}^{1}=p\left(t, e_{1}\right)$ and then inductively,

$$
\begin{aligned}
Z_{t}^{j} & =\operatorname{essinf}\left\{p\left(t, e_{j}+\sum_{i=1}^{j-1} y_{i} e_{i}\right)-\sum_{i=1}^{j-1} y_{i} Z_{t}^{i}: y_{1}, \ldots, y_{j-1} \in \mathbb{Q}\right\} \\
& =\inf \left\{p\left(t, e_{j}+\sum_{i=1}^{j-1} y_{i} e_{i}\right)-\sum_{i=1}^{j-1} y_{i} Z_{t}^{i}: y_{1}, \ldots, y_{j-1} \in \mathbb{Q}\right\} \quad \text { for } j \geq 2 .
\end{aligned}
$$

By (A.57), it is enough to show that $Z \in \mathcal{S}$ and

$$
p(t, y) \geq y Z_{t} \quad \text { for all } y \in \mathbb{Q}^{d} .
$$

The reason is that (A.57) and (A.60) imply that

$$
f(t, x+y)-f(t, x) \geq p(t, y) \geq y Z_{t} \quad \text { for all } y \in \mathbb{Q}^{d},
$$

which by the continuity of $f$ yields

$$
f(t, x+y)-f(t, x) \geq y Z_{t} \quad \text { for all } y \in \mathbb{R}^{d} .
$$

It is clear that $Z$ as the infimum of predictable processes is predictable. Furthermore, it follows from (A.58) and (A.59) that $p\left(t, y_{1} e_{1}\right) \geq y_{1} Z_{t}^{1}$ for all $y_{1} \in \mathbb{Q}$. Now assume that $Z_{t}^{1}, \ldots, Z_{t}^{j-1}$ are in $L^{0}(\mathcal{F})$ and

$$
p\left(t, \sum_{i=1}^{j-1} y_{i} e_{i}\right) \geq \sum_{i=1}^{j-1} y_{i} Z_{t}^{i} \quad \text { for all } y_{1}, \ldots, y_{j-1} \in \mathbb{Q} .
$$

Then one deduces from (A.58) and (A.59) that for all $y_{1}, \ldots, y_{j-1} \in \mathbb{Q}$ and $y_{j}>0$,

$$
p\left(t, y_{j} e_{j}+\sum_{i=1}^{j-1} y_{i} e_{i}\right)-\sum_{i=1}^{j-1} y_{i} Z_{t}^{i}=y_{j}\left[p\left(t, e_{j}+\sum_{i=1}^{j-1} \frac{y_{i}}{y_{j}} e_{i}\right)-\sum_{i=1}^{j-1} \frac{y_{i}}{y_{j}} Z_{t}^{i}\right] \geq y_{j} Z_{t}^{j} .
$$

Moreover, it follows from (A.61) that for all $v_{1}, \ldots, v_{j-1} \in \mathbb{Q}$,

$$
p\left(t, e_{j}+\sum_{i=1}^{j-1} y_{i} e_{i}\right)+p\left(t,-e_{j}+\sum_{i=1}^{j-1} v_{i} e_{i}\right) \geq p\left(t, \sum_{i=1}^{j-1}\left(y_{i}+v_{i}\right) e_{i}\right) \geq \sum_{i=1}^{j-1}\left(y_{i}+v_{i}\right) Z_{t}^{i}
$$

and therefore,

$$
p\left(t, e_{j}+\sum_{i=1}^{j-1} y_{i} e_{i}\right)-\sum_{i=1}^{j-1} y_{i} Z_{t}^{i} \geq-p\left(t,-e_{j}+\sum_{i=1}^{j-1} v_{i} e_{i}\right)+\sum_{i=1}^{j-1} v_{i} Z_{t}^{i} .
$$

Hence,

$$
Z_{t}^{j} \geq-p\left(t,-e_{j}+\sum_{i=1}^{j-1} v_{i} e_{i}\right)+\sum_{i=1}^{j-1} v_{i} Z_{t}^{i}
$$


and one obtains for $y_{j}<0$,

$$
p\left(t, y_{j} e_{j}+\sum_{i=1}^{j-1} y_{i} e_{i}\right)-\sum_{i=1}^{j-1} y_{i} Z_{t}^{i}=\left|y_{j}\right|\left[p\left(t,-e_{j}+\sum_{i=1}^{j-1} \frac{y_{i}}{\left|y_{j}\right|} e_{i}\right)-\sum_{i=1}^{j-1} \frac{y_{i}}{\left|y_{j}\right|} Z_{t}^{i}\right] \geq y_{j} Z_{t}^{j} .
$$

This shows that $Z_{t}^{j}$ is in $L^{0}(\mathcal{F})$ and

$$
p\left(t, \sum_{i=1}^{j} y_{i} e_{i}\right) \geq \sum_{i=1}^{j} y_{i} Z_{t}^{i} \quad \text { for all } y_{1}, \ldots, y_{j} \in \mathbb{Q} .
$$

The lemma now follows by induction. The second part of the lemma is seen by replacing $x$ by $X$.

\section{References}

[1] Aït-Sahalia, Y., J. Cacho-Diaz and T. Hurd (2009). Portfolio choice with jumps: a closed form solution. Annals of Applied Probability 19, 556-584.

[2] Artzner, Ph., F. Delbaen, J.-M. Eber and D. Heath (1999). Coherent measures of risk. Mathematical Finance 9, 203-228.

[3] Barles, G. And R. Buckdahn and E. Pardoux (1997). Backward stochastic differential equations and integral-partial differential equations. Stochastics and Stochastics Reports 60, 57-84, Taylor \& Francis.

[4] Becherer, D. (2006). Bounded solutions to backward SDE's with jumps for utility optimization and indifference hedging. Annals of Applied Probability 16, 2027-2054.

[5] Bender, C. And J. Steiner (2005). Least-Squares Monte Carlo for Backward SDEs. Annals of Applied Probability 15, 2172-2202.

[6] Ben-TAL, A. (1985). The entropic penalty approach to stochastic programming. Mathematics of Operations Research 10, 263-279.

[7] Bismut, J.-M. (1976). Théorie Probabiliste du Contrôle des Diffusions. American Mathematical Society, Providence, RI.

[8] Björk, T. AND I. Slinko (2006). Towards a general theory of good-deal bounds. Review of Finance 10, 221-260.

[9] Bordigoni, G., A. Matoussi And M. Schweizer (2007). A stochastic control approach to a robust utility maximization problem. In: Benth, F.E., G. Di Nunno, T. Lindstrom, B. Oksendal and T. Zhang (Eds.). Stochastic Analysis and Applications. Abel Symposium 2005. 125-151. Springer, Berlin.

[10] Carmona, R. (Ed.) (2009). Indifference Pricing: Theory and Applications. Princeton University Press, Princeton.

[11] Chen, Z. And L.G. Epstein (2002). Ambiguity, risk, and asset returns in continuous time. Econometrica 70, 1403-1443.

[12] Cheridito, P. AND Y. Hu (2010). Optimal consumption and investment in incomplete markets with general constraints. Mimeo, Princeton University.

[13] Cheridito, P. And M. Stadje (2010). BS $\Delta$ Es and BSDEs with non-Lipschitz drivers: comparison, convergence and robustness. Mimeo, Princeton University.

[14] Cheridito, P. And M. Stadje (2011). Existence, minimality and approximation of solutions to BSDEs with convex drivers. Stochastic Processe and Their Applications, forthcoming.

[15] Cont, R. and R. Tankov (2004). Financial Modelling with Jump Processes. Chapman \& Hall.

[16] Csiszár, I. (1975). I-divergence geometry of probability distributions and minimization problems. Annals of Probability 3, 146-158.

[17] Cvitanic, J. and I. Karatzas (1992). Convex duality in constrained portfolio optimization. Annals of Applied Probability 2, 767-818. 
[18] Delbaen, F., P. Grandits, T. Rheinländer, D. Samperi, M. Schweizer and C. Stricker (2002). Exponential hedging and entropic penalties. Mathematical Finance 12, 99-123.

[19] Delbaen, F., Y. Hu And X. Bao (2011). Backward SDEs with superquadratic growth. Probability Theory and Related Fields 150, 145-192.

[20] Delbaen, F., S. Peng and E. Rosazza Gianin (2010). Representation of the penalty term of dynamic concave utilities. Finance and Stochastics 14, 449-472.

[21] Delong, L. (2011). No-good-deal, local mean-variance and ambiguity risk pricing and hedging for an insurance payment process. Mimeo, Warsaw School of Economics.

[22] Duffie, D. And L.G. Epstein (1992). Stochastic differential utility. Econometrica 60, 353-394.

[23] El Karoui, N., S. Peng and M.C. Quenez (1997). Backward stochastic differential equations in finance. Mathematical Finance 7, 1-71.

[24] El Karoui, N. And R. Rouge (2000). Pricing via utility maximization and entropy. Mathematical Finance 10, 259-276.

[25] Ellsberg, D. (1961). Risk, ambiguity and the Savage axioms. Quarterly Journal of Economics 75, 643-669.

[26] Föllmer, H. AND A. Schied (2002). Convex measures of risk and trading constraints. Finance and Stochastics 6, 429-447.

[27] Föllmer, H. And A. Schied (2004). Stochastic Finance. 2nd ed., De Gruyter, Berlin.

[28] Föllmer, H., A. Schied And S. Weber (2009). Robust preferences and robust portfolio choice. In: Ciarlet, P., A. Bensoussan and Q. Zhang (Eds.). Mathematical Modelling and Numerical Methods in Finance. Handbook of Numerical Analysis 15, 29-88, Elsevier.

[29] Frittelli, M. and E. Rosazza Gianin (2002). Putting order in risk measures. Journal of Banking E6 Finance 26, 1473-1486.

[30] Gilboa, I. and D. Schmeidler (1989). Maxmin expected utility with non-unique prior. Journal of Mathematical Economics 18, 141-153.

[31] Gollier, C. (2001). The Economics of Risk and Time. MIT Press, Cambridge.

[32] Goovaerts, M.J., R. Kaas, R.J.A. Laeven and Q. Tang (2004). A comonotonic image of independence for additive risk measures. Insurance: Mathematics and Economics 35, 581-594.

[33] Hamadène, S. And M. Jeanblanc (2007). On the starting and stopping problem: application in reversible investments. Mathematics of Operations Research 32, 182-192.

[34] Hansen, L.P. And T.J. Sargent (2001). Robust control and model uncertainty. American Economic Review 91, 60-66.

[35] Hansen, L.P. and T.J. Sargent (2007). Robustness. Princeton University Press, Princeton.

[36] Horst, U. ANd M. Müller (2007). On the spanning property of risk bonds priced by equilibrium. Mathematics of Operations Research 32, 784-807.

[37] Horst, U., Y. Hu, P. Imkeller, A. RÉveillac and J. Zhang (2011). Forward-backward systems for expected utility maximization. Mimeo, Humboldt University, Berlin.

[38] Hu, Y., P. Imkeller And M. Müller (2005). Utility maximization in incomplete markets. Annals of Applied Probability 15, 1691-1712.

[39] Huber, P.J. (1981). Robust Statistics. Wiley, New York.

[40] Jacod, J. And A.N. Shiryaev (2003). Limit Theorems for Stochastic Processes. Springer, Berlin.

[41] Jeanblanc, M., A. Matoussi and A. Ngoupeyou (2010). Robust utility maximization in a discontinuous filtration. Mimeo, University of Evry-Val d'Essonne, Évry.

[42] Kabanov, Y. AND C. STRICKER (2002). On the optimal portfolio for the exponential utility maximization: remarks to the six-author paper. Mathematical Finance 12, 125-134.

[43] Kallsen, J. (2000). Optimal portfolios for exponential Lévy processes. Mathematical Methods of Operations Research 51, 357-374.

[44] Kazamaki, N. (1979). Transformation of $H^{p}$-martingales by a change of law. Probability Theory and Related Fields 46, 343-349.

[45] KlÖppel, S. ANd M. Schweizer (2007). Dynamic indifference valuation via convex risk measures. Mathematical Finance 17, 599-627. 
[46] Kobylanski, M. (2000). Backward stochastic differential equations and partial differential equations with quadratic growth. Annals of Probability 28, 259-276.

[47] Laeven, R.J.A. And M.A. Stadje (2011). Entropy coherent and entropy convex measures of risk. Mimeo, University of Amsterdam and Tilburg University.

[48] Lazrak, A. AND M.C. Quenez (2003). A generalized stochastic differential utility. Mathematics of Operations Research 28, 154-180.

[49] Lim, A.E.B. (2004). Quadratic hedging and mean-variance portfolio selection with random parameters in an incomplete market. Mathematics of Operations Research 29, 132-161.

[50] Lim, A.E.B. (2006). Mean-variance hedging when there are jumps. SIAM Journal on Control and Optimization 44, 1893-1922.

[51] Maccheroni, F., M. Marinacci And A. Rustichini (2006). Ambiguity aversion, robustness, and the variational representation of preferences. Econometrica 74, 1447-1498.

[52] Maenhout, P.J. (2004). Robust portfolio rules and asset pricing. Review of Financial Studies 17, 951-983.

[53] Mania, M. And M. Schweizer (2005). Dynamic exponential utility indifference valuation. Annals of Applied Probability 15, 2113-2143.

[54] Merton, R.C. (1969). Lifetime portfolio selection under uncertainty: the continuous-time case. Review of Economics and Statistics 51, 247-257.

[55] Merton, R.C. (1971). Optimum consumption and portfolio rules in a continuous-time model. Journal of Economic Theory 3, 373-413.

[56] Merton, R.C. (1976). Option pricing when underlying stock returns are discontinuous. Journal of Financial Economics 3, 125-144.

[57] Morlais, M.A. (2009). Quadratic BSDEs driven by a continuous martingale and applications to the utility maximization problem. Finance and Stochastics 13, 121-150.

[58] Morlais, M.A. (2010). A new existence result for BSDEs with jumps and application to the utility maximization problem. Stochastic Processes and Their Applications, forthcoming.

[59] Müller, M. (2005). Market Completion and Robust Utility Maximization. Dissertation, HumboldtUniversität zu Berlin.

[60] Øksendal, B. And A. Sulem (2011). A portfolio optimization under model uncertainty and BSDE games. Quantitative Finance 11, 1665-1674.

[61] OWARI, K. (2009). Robust exponential hedging in a Brownian setting. Mimeo, Hitotsubashi University.

[62] Pardoux, E. And S. Peng (1990). Adapted solution of a backward stochastic differential equation. Systems and Control Letters 14, 55-61.

[63] Protter, P.E. (2004). Stochastic Integration and Differential Equations. Springer, Berlin.

[64] Rockafellar, R.T. (1997) Convex Analysis. Princeton University Press, Princeton.

[65] Royer, M. (2006). Backward stochastic differential equations with jumps and related non-linear expectations. Stochastic Processes and Their Applications 116, 1358-1376.

[66] Rubinstein, M.E. (2004). Great moments in financial economics: III. short-sales and stock prices. Journal of Investment Management 2, 16-31.

[67] Ruszczyński, A. And A. Shapiro (2006). Optimization of convex risk functions. Mathematics of Operations Research 31, 433-452.

[68] Savage, L.J. (1954). The Foundations of Statistics. Wiley, New York (2nd ed. 1972, Dover, New York).

[69] Schachermayer, W. (2004). Utility Maximisation in Incomplete Markets. Lecture Notes in Mathematics 1856, 225-288, Springer, Berlin.

[70] Schied, A. (2006). Risk measures and robust optimization problems. Stochastic Models 22, 753-831.

[71] Sircar, R. and S. Sturm (2011). From smile asymptotics to market risk measures. Mimeo, Princeton University.

[72] Skiadas, C. (2003). Robust control and recursive utility. Finance and Stochastics 7, 475-489.

[73] Wald, A. (1950). Statistical Decision Functions. Wiley, New York. 
Figure 1: Numerical results for Example 2 of Subsection 6.1.2

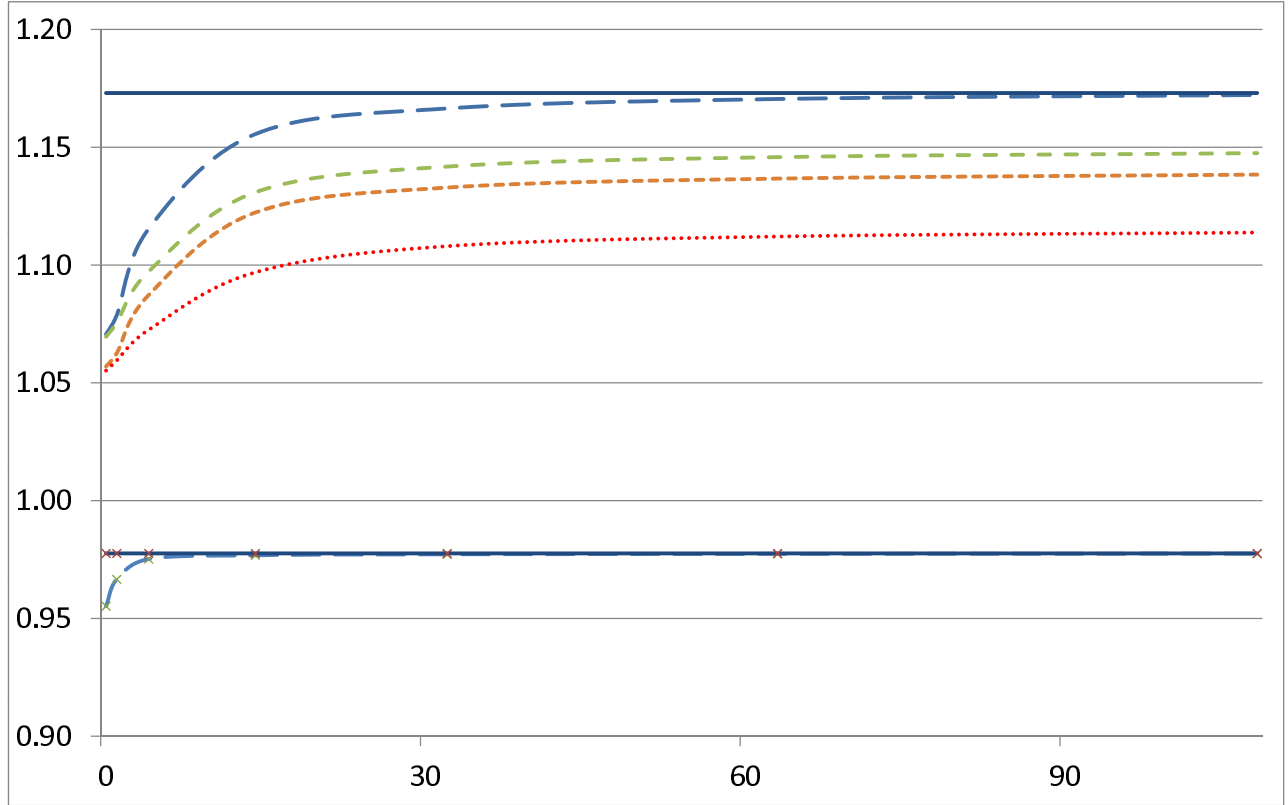

This figure plots $Y_{0}$ as a function of $\gamma$, in the setting of Example 2 in Subsection 6.1.2, for a European put option with strike price 2 and time-to-maturity of 0.5 years. The parameter values are $b=0.04, \sigma=0.2, a=1, \beta=$ $0.03, u_{\text {upper }}=10$ and $u_{\text {lower }}=0$. The number of simulations is 10,000 . We consider subsequently:

(i) no ambiguity $\left(\lambda=d_{+}=d_{-}=0\right)$, no hedge (long dashes with cross);

(ii) no ambiguity $\left(\lambda=d_{+}=d_{-}=0\right)$, with hedge (long dashes);

(iii) Brownian ambiguity only $\left(\lambda=0.05, d_{+}=d_{-}=0\right)$, with hedge (dashes);

(iv) jump ambiguity only $\left(\lambda=0, d_{+}=0.5, d_{-}=-0.25\right)$, with hedge (short dashes);

(v) both Brownian ambiguity and jump ambiguity $\left(\lambda=0.05, d_{+}=0.5, d_{-}=-0.25\right)$, with hedge (dots); and the asymptotes:

(vi) no ambiguity $\left(\lambda=d_{+}=d_{-}=0\right)$, risk neutrality (asymptote $\gamma=\infty$ ), no hedge (solid line with cross);

(vii) no ambiguity $\left(\lambda=d_{+}=d_{-}=0\right.$ ), risk neutrality (asymptote $\gamma=\infty$ ), with hedge (solid line). 